\title{
ECONOMICS
}

\section{INVESTMENT AND TECHNICAL PROGRESS IN THE G7 COUNTRIES AND AUSTRALIA}

by

John Pawley

and

Ernst Juerg Weber

Business School

The University of Western Australia 


\title{
INVESTMENT AND TECHNICAL PROGRESS IN THE G7 COUNTRIES AND AUSTRALIA
}

\author{
John Pawley* \\ and \\ Ernst Juerg Weber \\ Business School \\ University of Western Australia \\ Crawley, WA 6009 \\ Australia
}

May 2011

DISCUSSION PAPER 11.10

* This paper extends the research that John Pawley (2010) conducted for his Honours thesis at the University of Western Australia. The financial support of the C.A. Vargovic Memorial Fund and the UWA Business School is gratefully acknowledged. Corresponding author: juerg.weber@uwa.edu.au. 


\section{Abstract:}

The vintage model of capital accumulation predicts that technical progress depends on the installation of new capital equipment. In this paper it is found that investment raises labor productivity in the G7 countries and Australia. This finding implies that the decline in investment during the global financial crisis will have a long lasting detrimental effect on labor productivity and hence wages. 
Standard macroeconomic models imply that economic growth can be achieved either through capital accumulation or technical progress. Capital accumulation produces an ascending movement along the neoclassical production function and technical progress shifts the production function upwards. In growth accounting capital accumulation and technical progress are also viewed as independent sources of economic growth. Technical progress is the residual of output growth that cannot be explained by the growth in input factors, including among others capital. Yet, the widely accepted dichotomy between capital accumulation and technical progress as distinct sources of economic growth does not do justice to the diffusion of technology in the process of economic growth and development. Half a century ago, Johansen (1959), Salter (1955/1960), Solow (1960), and Kaldor and Mirrlees (1962) recognized that capital accumulation and technical progress are closely associated in the process of economic growth. Capital accumulation is a prerequisite for technical progress because, as Johansen put it, “new production techniques can be introduced only by means of new capital equipment." The idea that technical progress requires investment in new capital equipment to become effective is the cornerstone of the vintage model of capital accumulation. Since the business cycle is driven by changes in investment expenditure, an important prediction of the vintage model is that technical progress is cyclical.

As the vintage model of capital accumulation plays only a peripheral role in contemporary macroeconomics thinking, it is worthwhile to consider its origin and elaborate briefly on it. Writing his Ph.D. thesis at the University of Cambridge, W.E.G. Salter was the first to work seriously on the vintage model of capital accumulation. ${ }^{1}$ He submitted his Ph.D. thesis in 1955 and he published Productivity and Technical Change, which builds on his

\footnotetext{
${ }^{1}$ Weber (2011) discusses Salter's contributions to production theory and international trade theory.
} 
Ph.D. thesis, in 1960. Niehans (1993) opined that progress in economic theory follows a deterministic process because it is propelled by a drive for internal consistency. Each step is determined by the steps preceding it but creative minds can speed up the advance in economic theory along the largely preset path. Salter undoubtedly was a creative economist who advanced the understanding of the process of technical progress but the vintage model of capital accumulation would also have been discovered without him, albeit at a slower pace. Both Johansen $(1959,1961)$ and Solow $(1960)$ appear to have discovered the vintage model independently of Salter - Solow refers to Johansen but neither of them mentions Salter - and also Kaldor (1957) and Kaldor and Mirrlees (1962) worked independently on it.

In the opening chapter of Productivity and Technical Change, Salter (1960) observes that actual labor productivity lags behind best-practice productivity in the manufacturing industry. There is a delay in the adoption of new technology because it is worthwhile to use old machines as long as they earn a surplus over operating costs. Old machines can compete with new machines because old machines need only to cover operating costs, whereas the decision to install a new machine depends on its operating costs and capital costs. The capital costs of old machines are no longer relevant in the production decision because 'bygones are forever bygones'. Salter's vintage model of capital accumulation explains why different vintages of capital coexist side-by-side in industrial countries and - even more so - in developing ones where it is often difficult to find finance for the capital cost of new equipment. For the purpose of this article, the essential insight is that gross investment determines the speed at which new technology is adopted in the production process.

"Without gross investment, improving technology that requires new capital equipment simply represents a potential for higher productivity; to realize this potential requires gross investment. An economy with a low rate of gross investment is restricted in the rate at which new techniques can be brought into use; an economy with a high rate of gross investment can quickly bring new methods into use, and thus realize the benefits of improving technology. In this way, the rate 
of gross investment is a vital determinant of the extent to which observed productivity lags behind best-practice productivity.” (Salter 1960, pp. 63-64)

To begin with, Solow (1960) considers a Cobb-Douglas production function with a technical shift factor:

$$
Q(t)=A(t) L(t)^{\alpha} K(t)^{1-\alpha}
$$

Here, $L(t)$ and $K(t)$ measure the inputs of labor and physical capital at time $t$, and $Q(t)$ is output. An increase in total factor productivity $A(t)$ shifts the production function upwards over time. Solow comments that in the Cobb-Douglas production function technical progress is "peculiarly disembodied". The technical dimension of capital goods is perfectly malleable because no distinction is made between old and new capital goods. Therefore, all capital goods participate equally in technical progress - even museum pieces! Solow then puts forward the opposite viewpoint that capital goods of a certain vintage embody a fixed level of technology that cannot be changed at a later date. In this situation, the diffusion of technical progress requires the installation of new machinery that replaces obsolete equipment.

"The striking assumption is that old and new capital equipment participate equally in technical change. This conflicts with the casual observation that many if not most innovations need to be embodied in new kinds of durable equipment before they can be made effective. Improvements in technology affect output only to the extent that they are carried into practice either by net capital formation or by the replacement of old-fashioned equipment with the latest models, with a consequent shift in the distribution of equipment by date of birth.” (Solow 1960, p. 91)

The discovery of the vintage model of capital accumulation coincided with a shift from consumption to the production of armaments during World War II, which created favorable conditions for research in production theory. The activity analysis of Koopmans (1951a,b; 1957) would have provided an excellent starting point for the analysis of technical progress. In fact, Salter (1960, pp. 13-16) employs activity analysis in an informal way. There is no 
reason to believe that technical progress affects economic activities evenly across the economy. However, it took almost two decades until Atkinson and Stiglitz (1969) pointed out that technical progress produces an uneven upward-shift of the neoclassical production function, possibly affecting only a single point or activity on it. This long delay proves Niehans right: it requires creativity to make the next predetermined step in the largely deterministic process of scientific discovery in economics. The evolution of economic theory also includes many instances in which theories fell into oblivion, only to be rediscovered at a later date. Ricardian equivalence provides a famous example - the vintage model of capital accumulation is another. In the 1970s and 80s, the emphasis in macroeconomic research shifted back to consumption and the standard view on disembodied technical progress prevailed in growth theory. The vintage model of capital accumulation, which played no role in the development of endogenous growth models in the 1980s, did not live up to its potential until it was revived by Hulten (1992), Cooley et al. (1997) and Greenwood et al. (1997) in the 1990s.

This article deals with the empirical side of the vintage model of capital accumulation. Since technical progress is embodied in new machinery and equipment, the vintage model predicts that an increase in gross investment will lead to a long lasting improvement in labor productivity. Both the replacement of worn out machinery and the expansion of production facilities create opportunities to install new technology. The empirical analysis focuses on labor productivity because the vintage model does not support the notion of total factor productivity, which presumes disembodied technical progress. Salter (1960, p. 36) reasons that technical progress "raises the productivity of labour in two stages: the first is the direct effect of technical advances in each industry; and the second is the substitution of capital for labour, following upon the cheapening of capital goods relative to wages.” The price of 
capital goods falls relative to labor because technical progress makes it possible to produce commodities - including capital goods - with less labor and capital.

\section{Decomposition of Forecast-Error-Variances}

The empirical analysis is based on VAR models that include gross investment, aggregate output (GDP) and output per worker. Eight models with three variables and two lags are estimated using annual data for the G7 countries and Australia from 1950 to 2009. ${ }^{2}$ Following the original VAR methodology, which has never been abandoned by Sims (1980) and Doan (2007, p. 343), the logarithm of all variables is used because they grow exponentially but they are neither differenced nor detrended. The point is that it is important to preserve long-term relationships between variables because it may take some time until the full effect of investment on productivity is felt. ${ }^{3}$ In VAR models the identification of variable specific innovations requires an assumption on the causal structure of the contemporaneous correlation matrix of innovations. Here, it is assumed that the contemporaneous correlation between variable specific shocks represents a causal chain that runs from investment to GDP and to labor productivity. The effect of investment on GDP is suggested by the Keynesian multiplier, and the effect of GDP on labor productivity may reflect labor hoarding. The effect of investment on labor productivity, which arises in the vintage model of capital accumulation, is the main interest of this study. It should be noted that every VAR model is structural because the identification of variable specific shocks requires economic theory.

\footnotetext{
${ }^{2}$ Germany 1970-2009. The data are from the Penn World Table 7.0. Real GDP was constructed by multiplying real GDP per capita with population and investment equals 'investment share of real GDP per capita’ times real GDP per capita.

${ }^{3}$ The Johanson procedure indicates that variables are not cointegrated in most countries. All econometric work is included in an appendix of Economics Discussion Paper 11.10, Business School, University of Western Australia (available on SSRN, RePEc and the website of the UWA Business School). See Enders (2010, p. 303) for the continuing use of non-stationary VARs and Mills and Markellos (2008, pp. 375-378) for the impulse response asymptotics.
} 
The moving-average representation of a VAR model can be written in two ways, as decompositions of forecast-error-variances and as impulse responses. In this section, the forecast-error-variances for labor productivity are decomposed into the innovations experienced by each variable during the forecasting period. Labor productivity moves independently if its $k$-step ahead forecast-error-variances are caused by its own innovations, while labor productivity depends on other variables if most of the forecast-error-variances arise from shocks to those variables. In Table 1 the decomposition of the forecast-errorvariances is read along the rows, which add up to 100 . For example, $49.3 \%$ of the 10 -year ahead forecast-error-variance of Australian labor productivity is explained by shocks to investment, $43.3 \%$ by shocks to GDP and only $7.4 \%$ by shocks to labor productivity itself. Thus, Australian labor productivity is an endogenous variable that strongly depends on investment and GDP. The same pattern emerges in the other countries. At a forecasting horizon of 15 to 20 years, unforeseen investment shocks account for more than $80 \%$ of the forecast-error-variances in France and Japan; the share of investment lies between about 30\% and $60 \%$ in Germany, the United Kingdom, Italy and the USA; and it is less than $10 \%$ only in Canada. The large contributions of investment to the forecast-error-variances, which are found in Table 1, support the prediction of the vintage model of capital accumulation that investment is an important determinant of labor productivity.

In this section, it has been established that labor productivity depends strongly on investment in the G7 countries and Australia, except in Canada. The impulse response functions, which will be shown in the next section, confirm that - as implied by the vintage model of capital accumulation - investment in new capital goods has a long lasting positive effect on labor productivity. 
Table 1. Decomposition of Forecast-Error-Variances for Labor Productivity

\begin{tabular}{|c|c|c|c|}
\hline \multirow[b]{2}{*}{ Year } & Investment & GDP & $\begin{array}{c}\text { Labor } \\
\text { Productivity }\end{array}$ \\
\hline & \multicolumn{3}{|c|}{ Australia } \\
\hline 5 & 46.2 & 43.8 & 10.0 \\
\hline 10 & 49.3 & 43.3 & 7.4 \\
\hline 15 & 51.2 & 41.9 & 6.9 \\
\hline \multirow[t]{2}{*}{20} & 52.4 & 40.7 & 6.9 \\
\hline & \multicolumn{3}{|c|}{ Canada } \\
\hline 5 & 10.4 & 59.6 & 30.0 \\
\hline 10 & 8.2 & 63.9 & 27.9 \\
\hline 15 & 8.0 & 66.2 & 25.7 \\
\hline \multirow[t]{2}{*}{20} & 8.1 & 67.6 & 24.3 \\
\hline & \multicolumn{3}{|c|}{ France } \\
\hline 5 & 77.5 & 13.5 & 9.0 \\
\hline 10 & 82.5 & 8.3 & 9.2 \\
\hline 15 & 84.3 & 6.4 & 9.3 \\
\hline \multirow[t]{2}{*}{20} & 85.1 & 5.4 & 9.6 \\
\hline & \multicolumn{3}{|c|}{ Germany } \\
\hline 5 & 59.9 & 19.4 & 20.6 \\
\hline 10 & 43.4 & 24.9 & 31.7 \\
\hline 15 & 40.8 & 29.9 & 29.3 \\
\hline \multirow[t]{2}{*}{20} & 39.4 & 32.6 & 28.0 \\
\hline & \multicolumn{3}{|c|}{ Italy } \\
\hline 5 & 33.1 & 40.3 & 26.5 \\
\hline 10 & 44.9 & 32.8 & 22.2 \\
\hline 15 & 52.5 & 28.3 & 19.2 \\
\hline \multirow[t]{2}{*}{20} & 56.5 & 26.1 & 17.4 \\
\hline & \multicolumn{3}{|c|}{ Japan } \\
\hline 5 & 59.3 & 20.6 & 20.2 \\
\hline 10 & 76.1 & 11.8 & 12.1 \\
\hline 15 & 84.3 & 8.1 & 7.5 \\
\hline \multirow[t]{2}{*}{20} & 87.8 & 6.5 & 5.8 \\
\hline & \multicolumn{3}{|c|}{ United Kingdom } \\
\hline 5 & 30.1 & 47.0 & 22.9 \\
\hline 10 & 30.6 & 51.5 & 17.9 \\
\hline 15 & 30.4 & 55.1 & 14.5 \\
\hline \multirow[t]{2}{*}{20} & 29.8 & 57.6 & 12.6 \\
\hline & \multicolumn{3}{|c|}{ USA } \\
\hline 5 & 42.7 & 31.0 & 26.3 \\
\hline 10 & 40.9 & 26.8 & 32.3 \\
\hline 15 & 41.3 & 26.2 & 32.5 \\
\hline 20 & 42.0 & 26.9 & 31.2 \\
\hline
\end{tabular}




\section{Impulse Responses}

Figure 1 displays the responses of labor productivity to investment shocks in the G7 countries and Australia. The investment shocks are temporary, lasting a single year, and their size equals one standard deviation of the residuals of the investment equation for each country. Accordingly, the investment shocks range from $4.9 \%$ in Germany to $10.2 \%$ in Australia, with the other countries lying between these limits. ${ }^{4}$ The responses in labor productivity are plotted over 12 years along the lower axis and, since variables are logarithmic, the impulse responses are measured as percentage changes along the upper axis, using continuous growth compounding.

In Australia a temporary shock to investment immediately raises labor productivity by $1.8 \%$ and the productivity gain settles at $0.8 \%$ after about 10 years. The effect of investment on labor productivity is strongest in Japan and weakest in Canada. In Japan a shock to investment immediately raises productivity by $1.4 \%$ and the full effect of the investment shock is felt after about ten years, when the productivity gain reaches $4 \%$. In all countries productivity responds positively to investment, both in the short-term and long-term, except for Canada where it rises only in the short-term. The positive impulse responses of labor productivity to investment shocks are meaningful because, as shown by the decompositions of forecast-error-variances in Table 1 , investment accounts for a large share of the variation in labor productivity in all countries, except in Canada. As predicted by the vintage model of capital accumulation, a temporary increase in investment gives rise to a long lasting increase in labor productivity because investment creates opportunities to catch up with best practice productivity by installing new technology.

\footnotetext{
${ }^{4}$ The standard errors of the estimated investment equations are: Australia (10.2\%), Canada (7.9\%), France (6.5\%), Germany (4.9\%), Italy (7.2\%), Japan (7.7\%), United Kingdom (7.4\%) and United States (7.3\%).
} 
Figure 1. Responses of Labor Productivity in the G7 Countries and Australia

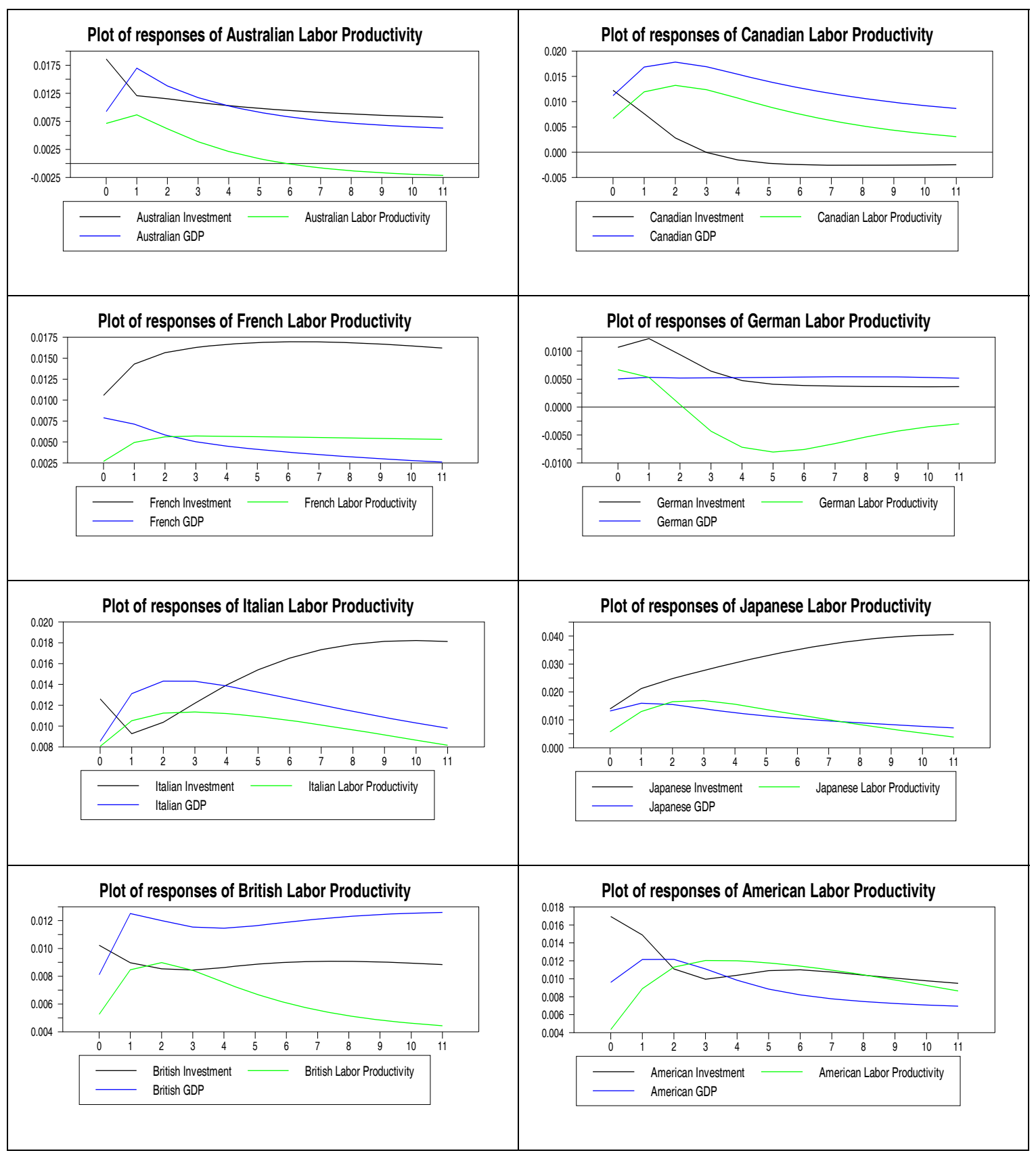


Besides the response of labor productivity to an investment shock, Figure 1 also displays the responses of productivity to GDP and to its own shocks. In all countries, a positive (negative) shock to GDP is followed by an increase (fall) in labor productivity, which may be explained by labor hoarding. Finally, the Keynesian multiplier prevails as an increase in investment raises GDP in all countries and there is evidence for an accelerator effect of GDP on investment in some countries (impulse responses not shown). ${ }^{5}$

\section{Conclusion}

One goal of this article was to unearth the origins of the vintage model of capital accumulation, which are not well known. The vintage model was independently discovered by Johansen (1959, 1961), Salter (1955/1960), Solow (1960), Kaldor (1957) and Kaldor and Mirrlees (1962), and it was rediscovered by Hulten (1992), Cooley et al. (1997) and Greenwood et al. (1997). The second goal was to demonstrate the empirical relevance of the vintage model. It was found that the dynamic interaction between investment, aggregate output and labor productivity supports the prediction of the vintage model that an increase in investment produces a long lasting improvement in labor productivity.

The vintage model of capital accumulation is still awaiting widespread application in macroeconomic analysis that would be commensurate with its significance. Indeed, the vintage model is of particular relevance in the current economic situation. Since investment is required for technical progress, the decline in investment during the global financial crisis will have a long lasting detrimental effect on labor productivity and hence wages in many countries.

\footnotetext{
${ }^{5}$ A VAR model with three variables produces nine impulse response functions, which gives 72 impulse response functions for eight countries.
} 


\section{References}

Atkinson, A.B. and Stiglitz, J. (1969) A New View of Technical Change, Economic Journal, 79/315, 573-578.

Cooley, T.F., Greenwood, J. and Yorukogly, M. (1997) The Replacement Problem, Journal of Monetary Economics, 40, 457-499.

Doan, T.A. (2007) RATS User’s Guide, Version 7, Evanston, IL: Estima.

Enders, W. (2010) Applied Econometric Time Series, New York: John Wiley.

Greenwood, J., Hercowitz, Z. and Krusell, P. (1997) Long-Run Implications of InvestmentSpecific Technological Change, American Economic Review, 87/3, 342-362.

Hulten, C.R. (1992) Growth Accounting when Technical Change is Embodied in Capital, American Economic Review, 82/4, 964-980.

Johansen, L. (1959) Substitution versus Fixed Production Coefficients in the Theory of Economic Growth: A Synthesis, Econometrica, 27/2, 157-176.

Johansen, L. (1961) A Method of Separating the Effects of Capital Accumulation and Shifts in Production Functions upon Growth in Labour Productivity, Economic Journal, 71, pp. 775-782.

Kaldor, N. (1957) A Model of Economic Growth, Economic Journal, 67/268, 591-624.

Kaldor, N. and Mirrlees, J.A. (1962) A New Model of Economic Growth, Review of Economic Studies, 29/3, 174-192. 
Koopmans, T.C. (1951) Analysis of Production as an Efficient Combination of Activities, in Koopmans, T.C. (ed.) Activity Analysis of Production and Allocation, Cowles Commission Monograph 13, New York: Wiley, Chapter. III.

Koopmans, T.C. (1951) Efficient Allocation of Resources, Econometrica, 19/4, 455-465.

Koopmans, T.C. (1957) Three Essays on the State of Economic Science, New York: McGraw-Hill.

Mills, T.C. and Markellos, R.N. (2008) The Econometric Modelling of Financial Time Series, $3^{\text {rd }}$ ed., Cambridge: Cambridge University Press.

Niehans, J. (1993) Revolution and Evolution in Economic Theory, Bateman Lecture, University of Western Australia, Australian Quarterly, 65/1, 498-515.

Pawley, J. (2010) Investment and Productivity in Australia, 1965-2007, Honours thesis, Business School, University of Western Australia.

Salter, W.E.G. (1960) Productivity and Technical Change, Cambridge: Cambridge University Press.

Sims, C.A. (1980) Macroeconomics and Reality, Econometrica, 48, 1-49.

Solow, R.M. (1960) Investment and Technical Progress, in Arrow, K.J., Karlin, S. and Suppes P. Mathematical Methods in the Social Sciences, Stanford: Stanford University Press.

Weber, E.J. (2011) Wilfred E.G. Salter: The Merits of a Classical Economic Education, History of Economics Review (forthcoming) 


\section{Econometric Work}

This appendix includes the estimated log-level VARS and VECMs.

\section{A) Log-Level VARs}

This section uses the program IMPULSES.PRG of RATS 7.2 (RATS User's Manual, Example 10.3).

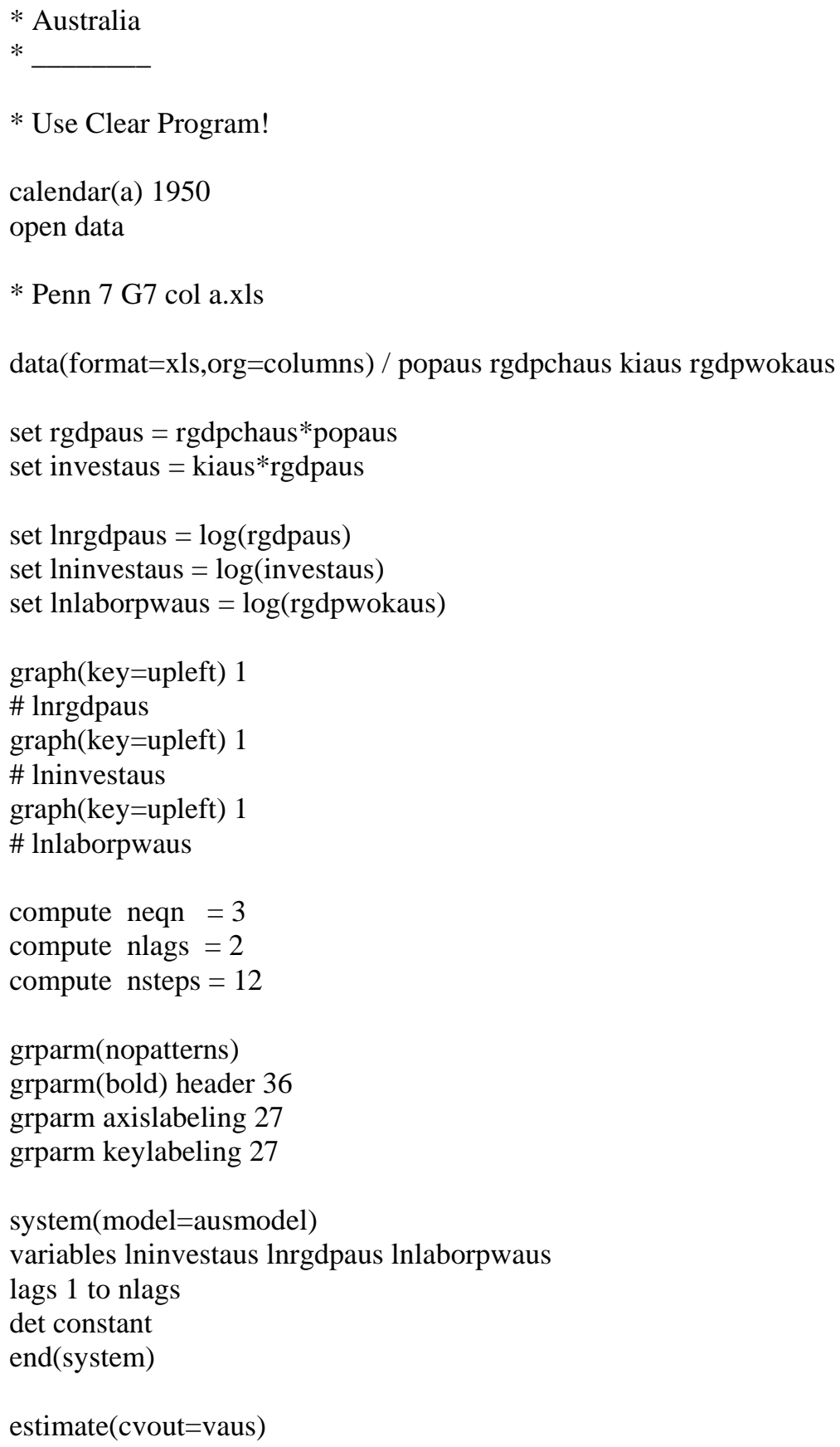


Dependent Variable LNINVESTAUS

Annual Data From 1952:01 To 2007:01

Usable Observations $56 \quad$ Degrees of Freedom 49

Mean of Dependent Variable $\quad 22.619144473$

Std Error of Dependent Variable $\quad 0.678313468$

Standard Error of Estimate $\quad 0.102463436$

Sum of Squared Residuals $\quad 0.5144390315$

Durbin-Watson Statistic $\quad 1.596253$

$\begin{array}{lllll}\quad \text { Variable } & \text { Coeff } & \text { Std Error } & \text { T-Stat } & \text { Signif } \\ * * * * * * * * * * * * * * * * * * * * * * * * * * * * * * * * * * * * * * * * * * * * * * * * * * * * * * * * * * * * * * * * * * * * * * * * * * * * * * & -1.12196 & 0.26734899 \\ \text { 1. LNINVESTAUS }\{1\} & -0.294435999 & 0.262430891 & -1.1969 \\ \text { 2. LNINVESTAUS }\{2\} & 0.822657267 & 0.257912486 & 3.18968 & 0.00248433 \\ \text { 3. LNRGDPAUS }\{1\} & 2.911846911 & 2.208487859 & 1.31848 & 0.19347285 \\ \text { 4. LNRGDPAUS }\{2\} & -2.806668840 & 2.124633909 & -1.32101 & 0.19263314 \\ \text { 5. LNLABORPWAUS }\{1\} & 1.840591529 & 1.898342355 & 0.96958 & 0.33701850 \\ \text { 6. LNLABORPWAUS }\{2\} & -0.930889029 & 1.885922166 & -0.49360 & 0.62379476 \\ \text { 7. Constant } & -1.194425099 & 1.164361610 & -1.02582 & 0.31001421\end{array}$

F-Tests, Dependent Variable LNINVESTAUS

Variable F-Statistic Signif

LNINVESTAUS $\quad 6.0473 \quad 0.0044952$

$\begin{array}{lll}\text { LNRGDPAUS } & 0.8750 & 0.4232542\end{array}$

$\begin{array}{lll}\text { LNLABORPWAUS } & 0.8461 & 0.4352699\end{array}$

Dependent Variable LNRGDPAUS

Annual Data From 1952:01 To 2007:01

Usable Observations $56 \quad$ Degrees of Freedom 49

Mean of Dependent Variable $\quad 19.517348909$

Std Error of Dependent Variable $\quad 0.622469495$

Standard Error of Estimate $\quad 0.022361522$

Sum of Squared Residuals $\quad 0.0245018447$

Durbin-Watson Statistic $\quad 1.837545$

$\begin{array}{lcccc}\text { Variable } & \text { Coeff } & \text { Std Error } & \text { T-Stat } & \text { Signif } \\ * * * * * * * * * * * * * * * * * * * * * * * * * * * * * * * * * * * * * * * * * * * * * * * * * * * * * * * * * * * * * * * * * * * * * * * * * * * * * * \\ \text { 1. LNINVESTAUS }\{1\} & -0.239238847 & 0.057272665 & -4.17719 & 0.00012091 \\ \text { 2. LNINVESTAUS }\{2\} & 0.206556879 & 0.056286572 & 3.66974 & 0.00059879 \\ \text { 3. LNRGDPAUS }\{1\} & 1.730833679 & 0.481978262 & 3.59110 & 0.00076093 \\ \text { 4. LNRGDPAUS }\{2\} & -0.689467614 & 0.463678057 & -1.48695 & 0.14343409 \\ \text { 5. LNLABORPWAUS }\{1\} & 0.231701410 & 0.414292406 & 0.55927 & 0.57852475 \\ \text { 6. LNLABORPWAUS }\{2\} & -0.246484665 & 0.411581836 & -0.59887 & 0.55201727 \\ \text { 7. Constant } & 0.107035809 & 0.254109156 & 0.42122 & 0.67543658\end{array}$

F-Tests, Dependent Variable LNRGDPAUS

Variable F-Statistic Signif

LNINVESTAUS $\quad 9.3977 \quad 0.0003511$

$\begin{array}{lll}\text { LNRGDPAUS } & 124.1011 & 0.0000000\end{array}$

$\begin{array}{lll}\text { LNLABORPWAUS } & 0.1804 & 0.8354664\end{array}$

Dependent Variable LNLABORPWAUS

Annual Data From 1952:01 To 2007:01

Usable Observations $56 \quad$ Degrees of Freedom 49

Mean of Dependent Variable $\quad 10.736281655$

Std Error of Dependent Variable $\quad 0.293666127$ 
Standard Error of Estimate

Sum of Squared Residuals

Durbin-Watson Statistic
0.023463688

0.0269766881

1.912711

$\begin{array}{lllll}\text { Variable } & \text { Coeff } & \text { Std Error } & \text { T-Stat } & \text { Signif } \\ * * * * * * * * * * * * * * * * * * * * * * * * * * * * * * * * * * * * * * * * * * * * * * * * * * * * * * * * * * * * * * * * * * * * * * * * * * * * * & \\ \text { 1. LNINVESTAUS }\{1\} & -0.226529717 & 0.060095550 & -3.76949 & 0.00044033 \\ \text { 2. LNINVESTAUS }\{2\} & 0.186532208 & 0.059060855 & 3.15831 & 0.00271677 \\ \text { 3. LNRGDPAUS }\{1\} & 0.598337509 & 0.505734260 & 1.18311 & 0.24247551 \\ \text { 4. LNRGDPAUS }\{2\} & -0.497854442 & 0.486532065 & -1.02327 & 0.31120493 \\ \text { 5. LNLABORPWAUS }\{1\} & 1.217935074 & 0.434712268 & 2.80170 & 0.00725868 \\ \text { 6. LNLABORPWAUS }\{2\} & -0.338486696 & 0.431868098 & -0.78377 & 0.43694548 \\ \text { 7. Constant } & 0.239319635 & 0.266633821 & 0.89756 & 0.37380969\end{array}$

F-Tests, Dependent Variable LNLABORPWAUS

$\begin{array}{lll}\text { Variable } & \text { F-Statistic } & \text { Signif } \\ \text { LNINVESTAUS } & 7.4572 & 0.0014881 \\ \text { LNRGDPAUS } & 1.3087 & 0.2794429 \\ \text { LNLABORPWAUS } & 11.8347 & 0.0000641\end{array}$

errors(model=ausmodel,steps=24,cv=vaus)

Decomposition of Variance for Series LNINVESTAUS

\begin{tabular}{rllll} 
Step & Std Error & \multicolumn{3}{l}{ LNINVESTAUS LNRGDPAUS LNLABORPWAUS } \\
1 & 0.09584577 & 100.000 & 0.000 & 0.000 \\
2 & 0.12242739 & 85.481 & 13.376 & 1.143 \\
3 & 0.13896128 & 81.109 & 17.458 & 1.433 \\
4 & 0.15029546 & 79.233 & 19.365 & 1.402 \\
5 & 0.15857057 & 78.219 & 20.479 & 1.301 \\
6 & 0.16489685 & 77.583 & 21.211 & 1.206 \\
7 & 0.16991688 & 77.130 & 21.731 & 1.139 \\
8 & 0.17402820 & 76.772 & 22.126 & 1.101 \\
9 & 0.17748846 & 76.468 & 22.441 & 1.091 \\
10 & 0.18047063 & 76.194 & 22.702 & 1.104 \\
11 & 0.18309420 & 75.940 & 22.927 & 1.134 \\
12 & 0.18544365 & 75.699 & 23.125 & 1.177 \\
13 & 0.18758002 & 75.468 & 23.302 & 1.230 \\
14 & 0.18954813 & 75.245 & 23.465 & 1.290 \\
15 & 0.19138145 & 75.029 & 23.616 & 1.356 \\
16 & 0.19310531 & 74.819 & 23.757 & 1.424 \\
17 & 0.19473912 & 74.616 & 23.890 & 1.494 \\
18 & 0.19629791 & 74.418 & 24.016 & 1.566 \\
19 & 0.19779342 & 74.226 & 24.137 & 1.637 \\
20 & 0.19923493 & 74.039 & 24.252 & 1.709 \\
21 & 0.20062981 & 73.858 & 24.363 & 1.779 \\
22 & 0.20198396 & 73.682 & 24.469 & 1.849 \\
23 & 0.20330213 & 73.511 & 24.572 & 1.917 \\
24 & 0.20458819 & 73.345 & 24.671 & 1.984
\end{tabular}

Decomposition of Variance for Series LNRGDPAUS

$\begin{array}{rlccc}\text { Step } & \text { Std Error } & \text { LNINVESTAUS } & \text { LNRGDPAUS } & \text { LNLABORPWAUS } \\ 1 & 0.02091729 & 79.208 & 20.792 & 0.000 \\ 2 & 0.03119292 & 54.628 & 45.093 & 0.279 \\ 3 & 0.03814126 & 50.615 & 49.199 & 0.187 \\ 4 & 0.04371769 & 49.910 & 49.807 & 0.283\end{array}$




$\begin{array}{ccccc}5 & 0.04854194 & 50.109 & 49.320 & 0.571 \\ 6 & 0.05288082 & 50.597 & 48.430 & 0.974 \\ 7 & 0.05687217 & 51.162 & 47.415 & 1.424 \\ 8 & 0.06059534 & 51.722 & 46.399 & 1.879 \\ 9 & 0.06410014 & 52.248 & 45.437 & 2.315 \\ 10 & 0.06742023 & 52.729 & 44.551 & 2.720 \\ 11 & 0.07057984 & 53.163 & 43.748 & 3.089 \\ 12 & 0.07359735 & 53.554 & 43.025 & 3.421 \\ 13 & 0.07648731 & 53.905 & 42.377 & 3.718 \\ 14 & 0.07926159 & 54.221 & 41.796 & 3.983 \\ 15 & 0.08193014 & 54.505 & 41.275 & 4.220 \\ 16 & 0.08450149 & 54.761 & 40.807 & 4.432 \\ 17 & 0.08698299 & 54.993 & 40.386 & 4.621 \\ 18 & 0.08938113 & 55.203 & 40.006 & 4.791 \\ 19 & 0.09170163 & 55.394 & 39.662 & 4.944 \\ 20 & 0.09394960 & 55.568 & 39.350 & 5.082 \\ 21 & 0.09612963 & 55.727 & 39.066 & 5.207 \\ 22 & 0.09824585 & 55.873 & 38.807 & 5.320 \\ 23 & 0.10030202 & 56.007 & 38.569 & 5.424 \\ 24 & 0.10230155 & 56.131 & 38.350 & 5.519\end{array}$

Decomposition of Variance for Series LNLABORPWAUS

$\begin{array}{rcccc}\text { Step } & \text { Std Error } & \text { LNINVSTAUS } & \text { LNRGDPAUS } & \text { LNLABORPWAUS } \\ 1 & 0.02194827 & 71.787 & 17.715 & 10.498 \\ 2 & 0.03146648 & 49.654 & 37.663 & 12.684 \\ 3 & 0.03675722 & 46.211 & 41.683 & 12.106 \\ 4 & 0.04026594 & 45.801 & 43.195 & 11.004 \\ 5 & 0.04285280 & 46.207 & 43.832 & 9.961 \\ 6 & 0.04490446 & 46.855 & 44.038 & 9.107 \\ 7 & 0.04662118 & 47.544 & 44.007 & 8.449 \\ 8 & 0.04811517 & 48.200 & 43.841 & 7.959 \\ 9 & 0.04945308 & 48.797 & 43.599 & 7.604 \\ 10 & 0.05067645 & 49.331 & 43.319 & 7.350 \\ 11 & 0.05181237 & 49.806 & 43.021 & 7.173 \\ 12 & 0.05287922 & 50.229 & 42.720 & 7.051 \\ 13 & 0.05388991 & 50.606 & 42.424 & 6.970 \\ 14 & 0.05485382 & 50.943 & 42.138 & 6.919 \\ 15 & 0.05577795 & 51.248 & 41.864 & 6.888 \\ 16 & 0.05666766 & 51.523 & 41.604 & 6.873 \\ 17 & 0.05752712 & 51.774 & 41.358 & 6.868 \\ 18 & 0.05835964 & 52.005 & 41.125 & 6.870 \\ 19 & 0.05916791 & 52.216 & 40.906 & 6.877 \\ 20 & 0.05995412 & 52.412 & 40.700 & 6.888 \\ 21 & 0.06072006 & 52.594 & 40.505 & 6.902 \\ 22 & 0.06146727 & 52.763 & 40.321 & 6.916 \\ 23 & 0.06219704 & 52.922 & 40.147 & 6.932 \\ 24 & 0.06291046 & 53.070 & 39.982 & 6.948\end{array}$

compute [vect[strings]] implabel=|| \$

"Australian Investment",\$

"Australian GDP",\$

"Australian Labor Productivity"||

@VARIRF(model=ausmodel,steps=nsteps,vlabels=implabel,byshocks,byvariables) 
* Canada

*

* Use Clear Program!

calendar(a) 1950

open data

* Penn 7 G7 col a.xls

data(format=xls,org=columns) / popcan rgdpchcan kican rgdpwokcan

set rgdpcan $=$ rgdpchcan*popcan

set investcan $=$ kican*rgdpcan

set lnrgdpcan = log(rgdpcan $)$

set lninvestcan $=\log ($ investcan $)$

set lnlaborpwcan = log(rgdpwokcan $)$

graph(key=upleft) 1

\# lnrgdpcan

graph(key=upleft) 1

\# lninvestcan

graph(key=upleft) 1

\# lnlaborpwcan

compute neqn $=3$

compute nlags $=2$

compute nsteps $=12$

grparm(nopatterns)

grparm(bold) header 36

grparm axislabeling 27

grparm keylabeling 27

system(model=canmodel)

variables Ininvestcan Inrgdpcan lnlaborpwcan

lags 1 to nlags

det constant

end(system)

estimate(cvout=vcan)

VAR/System - Estimation by Least Squares

Dependent Variable LNINVESTCAN

Annual Data From 1952:01 To 2007:01

Usable Observations $56 \quad$ Degrees of Freedom 49

Mean of Dependent Variable 22.952247596

Std Error of Dependent Variable $\quad 0.654620728$

Standard Error of Estimate $\quad 0.078537908$

Sum of Squared Residuals $\quad 0.3022419503$

Durbin-Watson Statistic $\quad 1.898783$

Variable Coeff Std Error T-Stat Signif 


$\begin{array}{lllll}\text { 1. LNINVESTCAN }\{1\} & 0.413290505 & 0.221814391 & 1.86323 & 0.06842953 \\ \text { 2. LNINVESTCAN }\{2\} & 0.096808346 & 0.242124238 & 0.39983 & 0.69101996 \\ \text { 3. LNRGDPCAN }\{1\} & -0.402502283 & 1.603566593 & -0.25100 & 0.80286032 \\ \text { 4. LNRGDPCAN }\{2\} & 0.771754948 & 1.549091036 & 0.49820 & 0.62057294 \\ \text { 5. LNLABORPWCAN }\{1\} & 2.222917026 & 1.357216772 & 1.63785 & 0.10785981 \\ \text { 6. LNLABORPWCAN }\{2\} & -1.721273508 & 1.202586382 & -1.43131 & 0.15868900 \\ \text { 7. Constant } & -1.525164571 & 1.216972265 & -1.25325 & 0.21606380\end{array}$

F-Tests, Dependent Variable LNINVESTCAN

Variable F-Statistic Signif

LNINVESTCAN $\quad 5.5913 \quad 0.0064977$

$\begin{array}{lll}\text { LNRGDPCAN } & 2.2934 & 0.1116621\end{array}$

LNLABORPWCAN $1.5265 \quad 0.2274507$

Dependent Variable LNRGDPCAN

Annual Data From 1952:01 To 2007:01

Usable Observations 56 Degrees of Freedom 49

Mean of Dependent Variable $\quad 20.062145596$

Std Error of Dependent Variable $\quad 0.575079637$

Standard Error of Estimate $\quad 0.018909522$

Sum of Squared Residuals $\quad 0.0175209307$

Durbin-Watson Statistic $\quad 1.914594$

\begin{tabular}{|c|c|c|c|c|}
\hline Varia & Coeff & Std Error & T-St & \\
\hline & & $* * * * * * * * * * * ;$ & $* * * * * * * * * * *$ & $* * * * * * * * * * * * * *$ \\
\hline 1. LNINVESTCAN $\{1\}$ & -0.147751979 & 0.053406109 & -2.76657 & 0.00796825 \\
\hline 2. LNINVESTCAN $\{2\}$ & 0.033947762 & 0.058296097 & 0.582 & 0.56301226 \\
\hline 3. LNRGDPCAN $\{1\}$ & 1.098714079 & 0.386089698 & 2.84575 & 0.00645163 \\
\hline 4. LNRGDPCAN $\{2\}$ & -0.026435743 & 0.372973654 & -0.07088 & 0.94378310 \\
\hline 5. LNLABORPWCAN $\{1\}$ & 0.493764912 & 0.326776210 & 1.51102 & 0.13720639 \\
\hline 6. LNLABORPWCAN $\{2\}$ & \multicolumn{4}{|c|}{$\begin{array}{llll}-0.367027404 & 0.289545951 & -1.26760 & 0.21093295\end{array}$} \\
\hline 7. Constant & -0.174480111 & 0.293009630 & -0.59548 & 0.55426605 \\
\hline
\end{tabular}

F-Tests, Dependent Variable LNRGDPCAN

Variable F-Statistic Signif

$\begin{array}{lll}\text { LNINVESTCAN } & 6.5200 & 0.0030857\end{array}$

$\begin{array}{lll}\text { LNRGDPCAN } & 281.1832 & 0.0000000\end{array}$

$\begin{array}{lll}\text { LNLABORPWCAN } & 1.4389 & 0.2470414\end{array}$

Dependent Variable LNLABORPWCAN

Annual Data From 1952:01 To 2007:01

Usable Observations $56 \quad$ Degrees of Freedom 49

Mean of Dependent Variable $\quad 10.783441991$

Std Error of Dependent Variable $\quad 0.208749923$

Standard Error of Estimate $\quad 0.019112144$

Sum of Squared Residuals $\quad 0.0178984292$

Durbin-Watson Statistic $\quad 1.985108$

$\begin{array}{lllll}\text { Variable } & \text { Coeff } & \text { Std Error } & \text { T-Stat } & \text { Signif } \\ * * * * * * * * * * * * * * * * * * * * * * * * * * * * * * * * * * * * * * * * * * * * * * * * * * * * * * * * * * * * * * * * * * * * * * * * * * * * * * * * * * * \\ \text { 1. LNINVESTCAN }\{1\} & -0.143018237 & 0.053978375 & -2.64955 & 0.01081924 \\ \text { 2. LNINVESTCAN }\{2\} & 0.052619880 & 0.058920762 & 0.89306 & 0.37618932 \\ \text { 3. LNRGDPCAN }\{1\} & -0.282350409 & 0.390226796 & -0.72355 & 0.47277937 \\ \text { 4. LNRGDPCAN }\{2\} & 0.368636660 & 0.376970207 & 0.97789 & 0.33293057 \\ \text { 5. LNLABORPWCAN }\{1\} & 1.791709324 & 0.330277741 & 5.42486 & 0.00000178\end{array}$




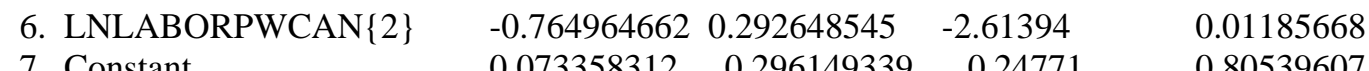

$\begin{array}{lllll}\text { 7. Constant } & 0.073358312 & 0.296149339 & 0.24771 & 0.80539607\end{array}$

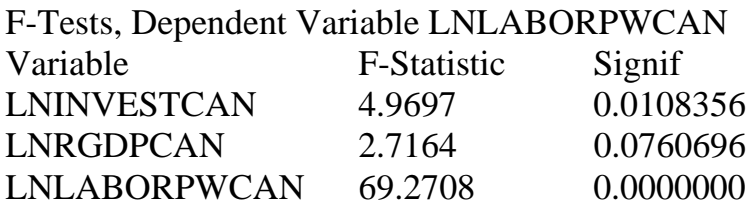

errors(model=canmodel,steps=24,cv=vcan)

Decomposition of Variance for Series LNINVESTCAN

\begin{tabular}{rllcc} 
Step & Std Error & \multicolumn{3}{l}{ LNINVESTCAN LNRGDPCAN LNLABORPWCAN } \\
1 & 0.07346549 & 100.000 & 0.000 & 0.000 \\
2 & 0.09352204 & 92.780 & 4.717 & 2.503 \\
3 & 0.10432748 & 83.327 & 11.044 & 5.629 \\
4 & 0.11143899 & 75.323 & 16.807 & 7.870 \\
5 & 0.11664597 & 69.260 & 21.612 & 9.128 \\
6 & 0.12074674 & 64.704 & 25.584 & 9.712 \\
7 & 0.12416625 & 61.189 & 28.905 & 9.906 \\
8 & 0.12714207 & 58.391 & 31.722 & 9.888 \\
9 & 0.12980870 & 56.100 & 34.139 & 9.761 \\
10 & 0.13224382 & 54.182 & 36.235 & 9.582 \\
11 & 0.13449417 & 52.548 & 38.070 & 9.382 \\
12 & 0.13658969 & 51.134 & 39.689 & 9.178 \\
13 & 0.13855103 & 49.895 & 41.128 & 8.977 \\
14 & 0.14039349 & 48.798 & 42.418 & 8.785 \\
15 & 0.14212911 & 47.818 & 43.579 & 8.603 \\
16 & 0.14376778 & 46.935 & 44.633 & 8.432 \\
17 & 0.14531787 & 46.135 & 45.592 & 8.272 \\
18 & 0.14678662 & 45.406 & 46.471 & 8.123 \\
19 & 0.14818033 & 44.738 & 47.278 & 7.984 \\
20 & 0.14950458 & 44.123 & 48.023 & 7.854 \\
21 & 0.15076431 & 43.555 & 48.712 & 7.733 \\
22 & 0.15196393 & 43.029 & 49.352 & 7.619 \\
23 & 0.15310741 & 42.539 & 49.947 & 7.513 \\
24 & 0.15419830 & 42.084 & 50.503 & 7.414
\end{tabular}

Decomposition of Variance for Series LNRGDPCAN

\begin{tabular}{rllcc} 
Step & Std Error & \multicolumn{3}{c}{ LNINVESTCAN LNRGDPCAN LNLABORPWCAN } \\
1 & 0.01768824 & 59.104 & 40.896 & 0.000 \\
2 & 0.02736228 & 38.426 & 60.132 & 1.443 \\
3 & 0.03495162 & 25.460 & 71.714 & 2.826 \\
4 & 0.04127100 & 18.302 & 78.209 & 3.489 \\
5 & 0.04667610 & 14.443 & 81.918 & 3.638 \\
6 & 0.05139485 & 12.323 & 84.139 & 3.538 \\
7 & 0.05558784 & 11.126 & 85.531 & 3.343 \\
8 & 0.05936664 & 10.436 & 86.435 & 3.128 \\
9 & 0.06280842 & 10.036 & 87.040 & 2.923 \\
10 & 0.06596777 & 9.807 & 87.455 & 2.738 \\
11 & 0.06888482 & 9.614 & 87.954 & 2.432 \\
13 & 0.07410907 & 9.586 & 88.107 & 2.307 \\
14 & 0.07646096 & 9.580 & 88.222 & 2.198 \\
15 & 0.07866296 & 9.588 & 88.310 & 2.102 \\
16 & 0.08072942 & 9.603 & 88.379 & 2.017
\end{tabular}




$\begin{array}{lllll}17 & 0.08267269 & 9.622 & 88.435 & 1.943 \\ 18 & 0.08450348 & 9.643 & 88.481 & 1.876 \\ 19 & 0.08623118 & 9.664 & 88.519 & 1.817 \\ 20 & 0.08786403 & 9.685 & 88.552 & 1.763 \\ 21 & 0.08940933 & 9.705 & 88.580 & 1.715 \\ 22 & 0.09087357 & 9.724 & 88.604 & 1.672 \\ 23 & 0.09226256 & 9.742 & 88.626 & 1.632 \\ 24 & 0.09358152 & 9.758 & 88.645 & 1.597\end{array}$

Decomposition of Variance for Series LNLABORPWCAN

\begin{tabular}{rcccc} 
Step & Std Error & \multicolumn{2}{c}{ LNINVESTCAN LNRGDPCAN LNLABORPWCAN } \\
1 & 0.01787777 & 46.992 & 39.148 & 13.861 \\
2 & 0.02834809 & 25.899 & 50.892 & 23.210 \\
3 & 0.03611678 & 16.566 & 55.713 & 27.720 \\
4 & 0.04174412 & 12.401 & 58.080 & 29.519 \\
5 & 0.04580431 & 10.411 & 59.589 & 30.000 \\
6 & 0.04878135 & 9.380 & 60.749 & 29.871 \\
7 & 0.05102209 & 8.809 & 61.721 & 29.469 \\
8 & 0.05275754 & 8.477 & 62.563 & 28.960 \\
9 & 0.05413857 & 8.280 & 63.298 & 28.422 \\
10 & 0.05526453 & 8.162 & 63.945 & 27.893 \\
11 & 0.05620237 & 8.094 & 64.516 & 27.390 \\
12 & 0.05699831 & 8.057 & 65.022 & 26.921 \\
13 & 0.05768506 & 8.040 & 65.473 & 26.487 \\
14 & 0.05828629 & 8.036 & 65.877 & 26.087 \\
15 & 0.05881940 & 8.040 & 66.241 & 25.719 \\
16 & 0.05929743 & 8.050 & 66.569 & 25.381 \\
17 & 0.05973029 & 8.062 & 66.868 & 25.069 \\
18 & 0.06012557 & 8.077 & 67.141 & 24.782 \\
19 & 0.06048921 & 8.093 & 67.392 & 24.515 \\
20 & 0.06082587 & 8.109 & 67.623 & 24.268 \\
21 & 0.06113925 & 8.125 & 67.836 & 24.038 \\
22 & 0.06143234 & 8.142 & 68.034 & 23.824 \\
23 & 0.06170756 & 8.157 & 68.219 & 23.623 \\
24 & 0.06196689 & 8.173 & 68.392 & 23.435
\end{tabular}

compute [vect[strings]] implabel=|| \$

"Canadian Investment",\$

"Canadian GDP",\$

"Canadian Labor Productivity"||

@VARIRF(model=canmodel,steps=nsteps,vlabels=implabel,byshocks,byvariables)

* France

*

Use clear program!

calendar(a) 1950

open data

* Penn 7 G7 col a.xls 


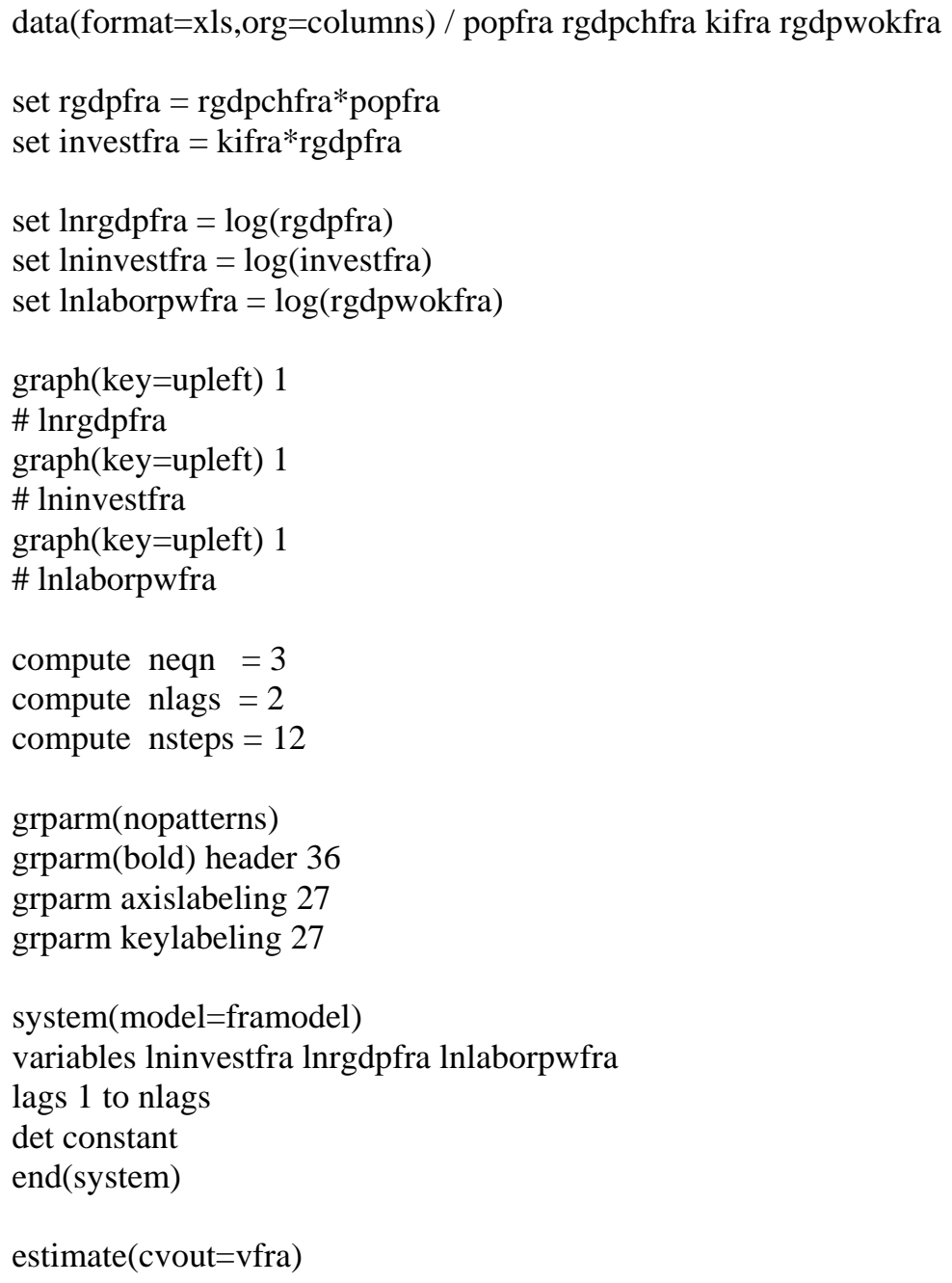

VAR/System - Estimation by Least Squares Dependent Variable LNINVESTFRA Annual Data From 1952:01 To 2007:01 Usable Observations 56 Degrees of Freedom 49 Mean of Dependent Variable 23.695671126 Std Error of Dependent Variable $\quad 0.575150889$ Standard Error of Estimate $\quad 0.064610236$ Sum of Squared Residuals $\quad 0.2045496502$ Durbin-Watson Statistic 2.090131

\begin{tabular}{|c|c|c|c|c|}
\hline Variable & & Std Error & T-Stat & Signif \\
\hline 1. LNINVESTFRA $\{1\}$ & 1218901534 & 0232533218 & 524184 & 00000 \\
\hline 2. LNI & 715336 & 0.203397139 & & 0.17231075 \\
\hline 3. LNRGDPFRA $\{1\}$ & -2.337043806 & 2.966269487 & -0.78787 & 0.43456587 \\
\hline 4. LNRGDPFRA $\{2\}$ & 2.229119214 & 2.921781149 & 0.76293 & 0.44916169 \\
\hline 5. LNLABORPWFRA $\{1\}$ & 1.979844235 & 3.121270140 & 0.63431 & 0.52883074 \\
\hline 6. LNLABORPWFRA $\{2\}$ & -1.796227014 & 3.086650961 & -0.58193 & 0.56327913 \\
\hline 7. Constant & 1.821625017 & 2.712313445 & 0.67161 & 0.50498430 \\
\hline
\end{tabular}

F-Tests, Dependent Variable LNINVESTFRA

Variable F-Statistic Signif 


$\begin{array}{lll}\text { LNINVESTFRA } & 31.8653 & 0.0000000 \\ \text { LNRGDPFRA } & 0.3384 & 0.7145812 \\ \text { LNLABORPWFRA } & 0.2620 & 0.7705854\end{array}$

Dependent Variable LNRGDPFRA

Annual Data From 1952:01 To 2007:01

Usable Observations $56 \quad$ Degrees of Freedom 49

Mean of Dependent Variable $\quad 20.706161945$

Std Error of Dependent Variable $\quad 0.541855446$

Standard Error of Estimate $\quad 0.015972665$

Sum of Squared Residuals $\quad 0.0125011751$

Durbin-Watson Statistic $\quad 2.040104$

$\begin{array}{lcccc}\text { Variable } & \text { Coeff } & \text { Std Error } & \text { T-Stat } & \text { Signif } \\ * * * * * * * * * * * * * * * * * * * * * * * * * * * * * * * * * * * * * * * * * * * * * * * * * * * * * * * * * * * * * * * * * * * * * * * * * * * * * * & \\ \text { 1. LNINVESTFRA }\{1\} & 0.082698666 & 0.057485862 & 1.43859 & 0.15662345 \\ \text { 2. LNINVESTFRA }\{2\} & -0.046519275 & 0.050282966 & -0.92515 & 0.35942204 \\ \text { 3. LNRGDPFRA }\{1\} & 0.292208159 & 0.733308389 & 0.39848 & 0.69200808 \\ \text { 4. LNRGDPFRA }\{2\} & 0.620698493 & 0.722310174 & 0.85932 & 0.39434640 \\ \text { 5. LNLABORPWFRA }\{1\} & 0.753305331 & 0.771626985 & 0.97626 & 0.33373301 \\ \text { 6. LNLABORPWFRA }\{2\} & -0.710392814 & 0.763068580 & -0.93097 & 0.35643414 \\ \text { 7. Constant } & 0.521820794 & 0.670526469 & 0.77823 & 0.44017790\end{array}$

F-Tests, Dependent Variable LNRGDPFRA

Variable $\quad$ F-Statistic Signif

LNINVESTFRA $\quad 1.2123 \quad 0.3062926$

$\begin{array}{lll}\text { LNRGDPFRA } & 94.4268 & 0.0000000\end{array}$

LNLABORPWFRA $\quad 0.5138 \quad 0.6014294$

Dependent Variable LNLABORPWFRA

Annual Data From 1952:01 To 2007:01

Usable Observations $56 \quad$ Degrees of Freedom 49

Mean of Dependent Variable $\quad 10.640874284$

Std Error of Dependent Variable $\quad 0.433429965$

Standard Error of Estimate $\quad 0.014369135$

Sum of Squared Residuals $\quad 0.0101171306$

Durbin-Watson Statistic $\quad 2.084967$

$\begin{array}{lllrl}\text { Variable } & \text { Coeff } & \text { Std Error } & \text { T-Stat } & \text { Signif } \\ * * * * * * * * * * * * * * * * * * * * * * * * * * * * * * * * * * * * * * * * * * * * * * * * * * * * * * * * * * * * * * * * * * * * * * * * * * * * * * & \\ \text { 1. LNINVESTFRA }\{1\} & 0.084715631 & 0.051714736 & 1.63813 & 0.10780042 \\ \text { 2. LNINVESTFRA }\{2\} & -0.050497005 & 0.045234953 & -1.11633 & 0.26972659 \\ \text { 3. LNRGDPFRA }\{1\} & -0.847273789 & 0.659690018 & -1.28435 & 0.20505831 \\ \text { 4. LNRGDPFRA }\{2\} & 0.785522785 & 0.649795936 & 1.20888 & 0.23251159 \\ \text { 5. LNLABORPWFRA }\{1\} & 1.842674953 & 0.694161728 & 2.65453 & 0.01068082 \\ \text { 6. LNLABORPWFRA }\{2\} & -0.832875432 & 0.686462520 & -1.21329 & 0.23083689 \\ \text { 7. Constant } & 0.391510980 & 0.603210906 & 0.64904 & 0.51934004\end{array}$

F-Tests, Dependent Variable LNLABORPWFRA

$\begin{array}{lll}\text { Variable } & \text { F-Statistic } & \text { Signif } \\ \text { LNINVESTFRA } & 1.4966 & 0.2339397 \\ \text { LNRGDPFRA } & 1.1186 & 0.3349493 \\ \text { LNLABORPWFRA } & 59.6251 & 0.0000000\end{array}$

errors(model=framodel,steps=24,cv=vfra) 
Decomposition of Variance for Series LNINVESTFRA

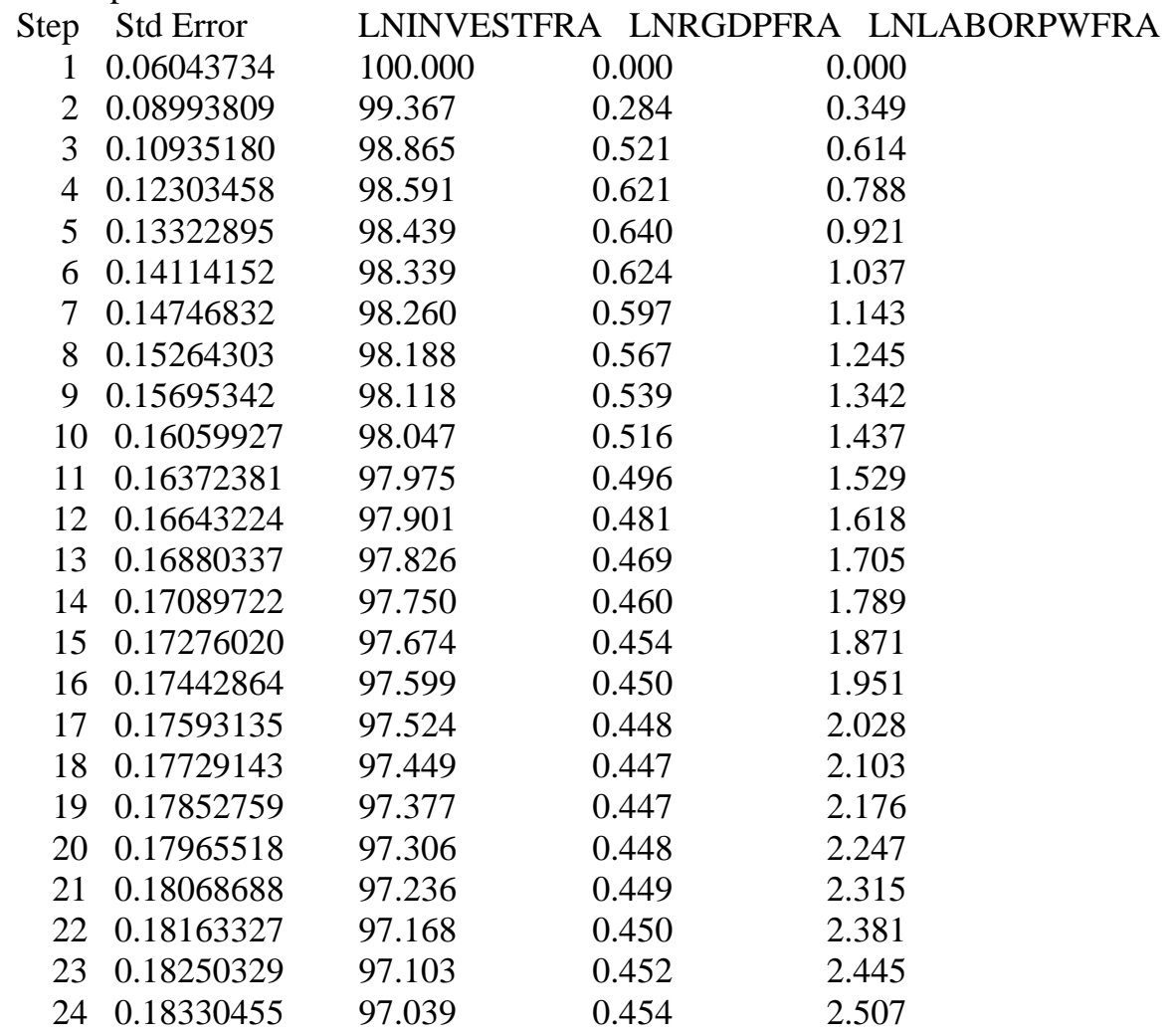

Decomposition of Variance for Series LNRGDPFRA

\begin{tabular}{rllll} 
Step & Std Error & \multicolumn{2}{c}{ LNINVESTFRA LNRGDPFRA } & LNLABORPWFRA \\
1 & 0.01494106 & 65.878 & 34.122 & 0.000 \\
2 & 0.02390196 & 73.345 & 25.940 & 0.715 \\
3 & 0.03108718 & 77.933 & 20.805 & 1.262 \\
4 & 0.03724223 & 80.951 & 17.471 & 1.578 \\
5 & 0.04272898 & 83.077 & 15.152 & 1.771 \\
6 & 0.04772672 & 84.659 & 13.435 & 1.906 \\
7 & 0.05233128 & 85.881 & 12.107 & 2.012 \\
8 & 0.05660043 & 86.850 & 11.047 & 2.103 \\
9 & 0.06057347 & 87.632 & 10.182 & 2.186 \\
10 & 0.06427977 & 88.271 & 9.466 & 2.263 \\
11 & 0.06774283 & 88.799 & 8.863 & 2.337 \\
12 & 0.07098228 & 89.239 & 8.352 & 2.410 \\
13 & 0.07401510 & 89.606 & 7.913 & 2.481 \\
14 & 0.07685629 & 89.915 & 7.533 & 2.551 \\
15 & 0.07951929 & 90.176 & 7.203 & 2.621 \\
16 & 0.08201629 & 90.396 & 6.913 & 2.690 \\
17 & 0.08435839 & 90.583 & 6.658 & 2.760 \\
18 & 0.08655581 & 90.740 & 6.431 & 2.829 \\
19 & 0.08861794 & 90.873 & 6.229 & 2.897 \\
20 & 0.09055345 & 90.985 & 6.049 & 2.966 \\
21 & 0.09237037 & 91.079 & 5.887 & 3.034 \\
22 & 0.09407612 & 91.157 & 5.741 & 3.102 \\
23 & 0.09567763 & 91.222 & 5.609 & 3.169 \\
24 & 0.09718131 & 91.275 & 5.489 & 3.235
\end{tabular}


Decomposition of Variance for Series LNLABORPWFRA

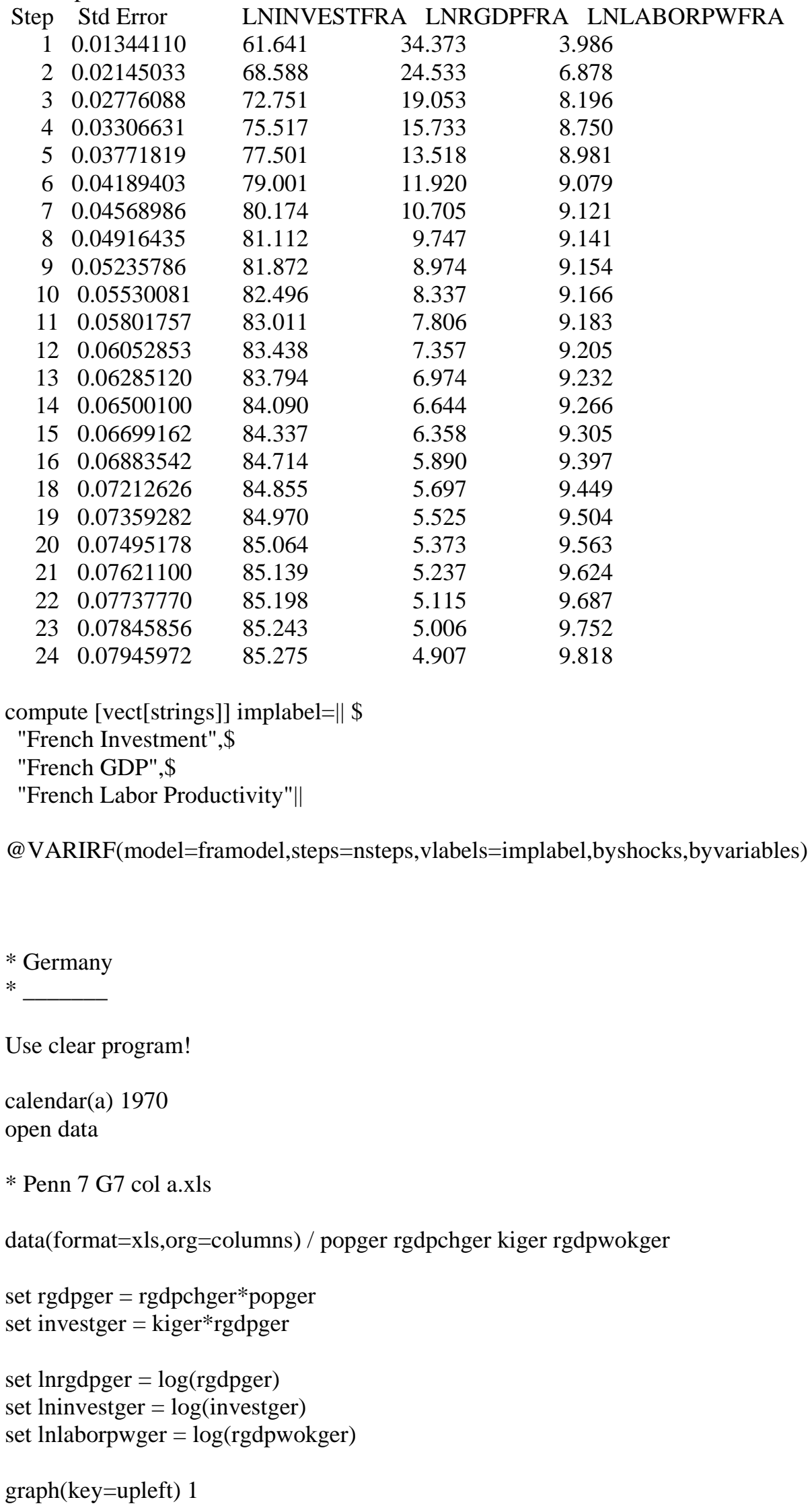




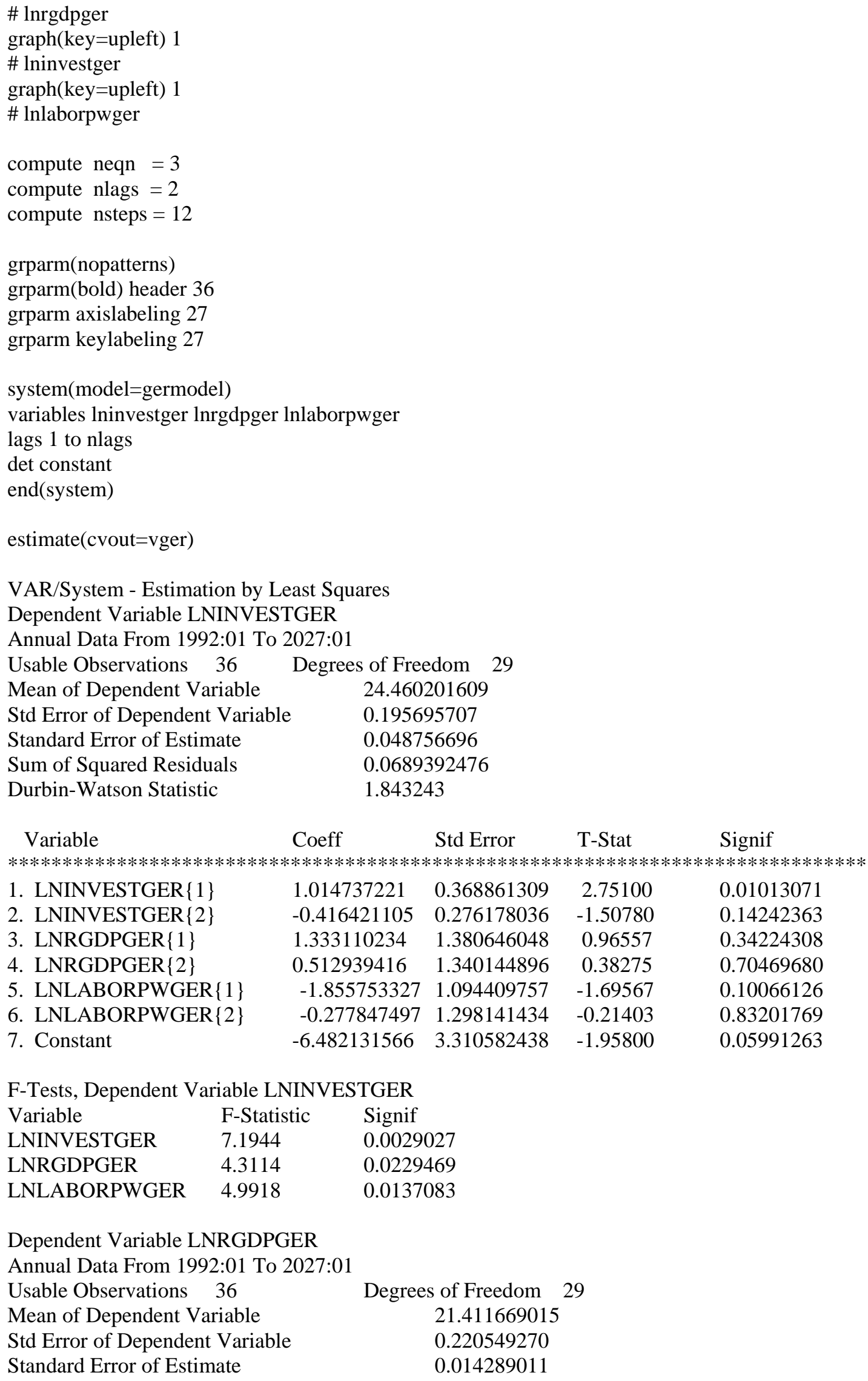

VAR/System - Estimation by Least Squares Dependent Variable LNINVESTGER

F-Tests, Dependent Variable LNINVESTGER

Variable $\quad$ F-Statistic Signif

LNINVESTGER $\quad 7.1944 \quad 0.0029027$

LNRGDPGER $\quad 4.3114 \quad 0.0229469$

LNLABORPWGER $4.9918 \quad 0.0137083$

Dependent Variable LNRGDPGER Annual Data From 1992:01 To 2027:01 Usable Observations 36 Mean of Dependent Variable Std Error of Dependent Variable Degrees of Freedom 29 21.411669015 0.220549270 Standard Error of Estimate 0.014289011 
Sum of Squared Residuals

Durbin-Watson Statistic
0.0059210993

1.880821

\begin{tabular}{|c|c|c|c|c|c|}
\hline \multicolumn{2}{|l|}{ Variable } & Coeff & $\begin{array}{l}\text { Std Error } \\
* * * * * * * * * * *\end{array}$ & T-Stat & $\begin{array}{l}\text { Signif } \\
k * * * * * * * * * * * * * *\end{array}$ \\
\hline \multicolumn{2}{|c|}{ 1. LNINVESTGER $\{1\}$} & 0.072931710 & 0.108101322 & 0.67466 & 0.50523478 \\
\hline \multicolumn{2}{|l|}{ 2. LNINVESTGER $\{2\}$} & -0.115632942 & 0.080938852 & -1.42865 & 0.16378914 \\
\hline \multicolumn{2}{|l|}{ 3. LNRGDPGER $\{1\}$} & 1.457161006 & 0.404622712 & 3.60128 & 0.00116687 \\
\hline \multicolumn{2}{|c|}{ 4. LNRGDPGER $\{2\}$} & -0.140374166 & 0.392753134 & -0.35741 & 0.72337241 \\
\hline \multicolumn{2}{|c|}{ 5. LNLABORPWGER $\{1\}$} & -0.480055088 & 0.320736111 & -1.49673 & 0.14526917 \\
\hline \multicolumn{2}{|c|}{ 6. LNLABORPWGER $\{2\}$} & 0.063405606 & 0.380443277 & 0.16666 & 0.86879229 \\
\hline \multicolumn{2}{|c|}{ 7. Constant } & -1.189958632 & 0.970224661 & -1.22648 & 0.22988455 \\
\hline \multicolumn{6}{|c|}{ F-Tests, Dependent Variable LNRGDPGER } \\
\hline Variable & \multicolumn{2}{|c|}{ F-Statistic Signif } & & & \\
\hline LNINVESTGER & \multicolumn{2}{|c|}{$2.3537 \quad 0.1129$} & 201 & & \\
\hline LNRGDPGER & \multicolumn{2}{|c|}{26.7648} & 003 & & \\
\hline LNLABORPWGER & \multicolumn{2}{|c|}{2.6571} & 761 & & \\
\hline
\end{tabular}

Dependent Variable LNLABORPWGER

Annual Data From 1992:01 To 2027:01

Usable Observations $36 \quad$ Degrees of Freedom 29

Mean of Dependent Variable $\quad 10.875261318$

Std Error of Dependent Variable $\quad 0.158643236$

Standard Error of Estimate $\quad 0.015129044$

Sum of Squared Residuals $\quad 0.0066377511$

Durbin-Watson Statistic $\quad 1.885574$

\begin{tabular}{|c|c|c|c|c|}
\hline Variable & Coeff & Std Error & T-Stat & Signif \\
\hline 1. LNINVESTGER $\{1\}$ & 0.033969296 & 0.114456462 & 0.29679 & 0.76874377 \\
\hline 2. LNINVESTGER $\{2\}$ & -0.068509701 & 0.085697144 & -0.79944 & 0.43053519 \\
\hline 3. LNRGDPGER $\{1\}$ & 0.201221669 & 0.428409968 & 0.46969 & 0.64208623 \\
\hline 4. LNRGDPGER $\{2\}$ & 0.253521643 & 0.415842593 & 0.60966 & 0.54683609 \\
\hline 5. LNLABORPWGER $\{1\}$ & 0.798001248 & 0.339591780 & 2.34988 & 0.02579709 \\
\hline 6. LNLABORPWGER $\{2\}$ & -0.411373115 & 0.402809055 & -1.02126 & 0.31557532 \\
\hline 7. Constant & -2.208154559 & 1.027262939 & -2.14955 & 0.04006657 \\
\hline
\end{tabular}

F-Tests, Dependent Variable LNLABORPWGER

$\begin{array}{lll}\text { Variable } & \text { F-Statistic } & \text { Signif } \\ \text { LNINVESTGER } & 0.9465 & 0.3997540 \\ \text { LNRGDPGER } & 2.7367 & 0.0815162 \\ \text { LNLABORPWGER } & 3.8427 & 0.0330851\end{array}$

errors(model=germodel,steps=24,cv=vger)

Decomposition of Variance for Series LNINVESTGER

\begin{tabular}{|c|c|c|c|c|}
\hline Step & Std Error & \multicolumn{3}{|r|}{ LNLABORPWGER } \\
\hline 1 & 0.04376047 & 100.000 & 0.000 & 0.000 \\
\hline 2 & 0.06013178 & 95.746 & 0.016 & 4.238 \\
\hline 3 & 0.06968944 & 80.029 & 0.018 & 19.953 \\
\hline 4 & 0.07971465 & 61.866 & 0.123 & 38.011 \\
\hline 5 & 0.08834384 & 50.379 & 0.358 & 49.263 \\
\hline 6 & 0.09372794 & 44.758 & 0.697 & 54.545 \\
\hline 7 & 0.09634054 & 42.371 & 1.125 & 56.505 \\
\hline
\end{tabular}




$\begin{array}{ccccc}8 & 0.09741755 & 41.477 & 1.611 & 56.912 \\ 9 & 0.09789402 & 41.154 & 2.116 & 56.391 \\ 11 & 0.09852972 & 40.913 & 3.051 & 56.036 \\ 12 & 0.09883779 & 40.856 & 3.452 & 55.691 \\ 13 & 0.09913352 & 40.830 & 3.810 & 55.360 \\ 14 & 0.09941646 & 40.819 & 4.131 & 55.051 \\ 15 & 0.09969385 & 40.804 & 4.422 & 54.775 \\ 16 & 0.09997194 & 40.773 & 4.689 & 54.538 \\ 17 & 0.10025161 & 40.725 & 4.938 & 54.337 \\ 18 & 0.10052919 & 40.663 & 5.173 & 54.164 \\ 19 & 0.10079947 & 40.594 & 5.396 & 54.011 \\ 20 & 0.10105818 & 40.523 & 5.609 & 53.868 \\ 21 & 0.10130308 & 40.456 & 5.813 & 53.731 \\ 22 & 0.10153374 & 40.394 & 6.009 & 53.597 \\ 23 & 0.10175096 & 40.337 & 6.197 & 53.466 \\ 24 & 0.10195606 & 40.286 & 6.376 & 53.338\end{array}$

Decomposition of Variance for Series LNRGDPGER

\begin{tabular}{rllcc} 
Step & Std Error & \multicolumn{2}{l}{ LNINVESTGER LNRGDPGER LNLABORPWGER } \\
1 & 0.01282478 & 74.770 & 25.230 & 0.000 \\
2 & 0.02062326 & 76.418 & 21.171 & 2.411 \\
3 & 0.02591010 & 69.010 & 20.628 & 10.362 \\
4 & 0.03033041 & 57.965 & 20.599 & 21.436 \\
5 & 0.03427913 & 48.600 & 20.786 & 30.614 \\
6 & 0.03752617 & 42.480 & 21.452 & 36.068 \\
7 & 0.04000951 & 38.916 & 22.593 & 38.491 \\
8 & 0.04188672 & 36.926 & 24.029 & 39.044 \\
9 & 0.04336453 & 35.816 & 25.563 & 38.622 \\
10 & 0.04460213 & 35.178 & 27.048 & 37.774 \\
11 & 0.04569675 & 34.798 & 28.403 & 36.798 \\
12 & 0.04670117 & 34.561 & 29.597 & 35.842 \\
13 & 0.04764297 & 34.398 & 30.628 & 34.974 \\
14 & 0.04853680 & 34.269 & 31.510 & 34.222 \\
15 & 0.04939039 & 34.147 & 32.263 & 33.590 \\
16 & 0.05020732 & 34.022 & 32.909 & 33.070 \\
17 & 0.05098856 & 33.889 & 33.468 & 32.642 \\
18 & 0.05173374 & 33.752 & 33.961 & 32.287 \\
19 & 0.05244228 & 33.615 & 34.400 & 31.985 \\
20 & 0.05311403 & 33.482 & 34.799 & 31.719 \\
21 & 0.05374964 & 33.358 & 35.164 & 31.478 \\
22 & 0.05435048 & 33.244 & 35.500 & 31.256 \\
23 & 0.05491844 & 33.139 & 35.813 & 31.048 \\
24 & 0.05545568 & 33.045 & 36.102 & 30.852
\end{tabular}

Decomposition of Variance for Series LNLABORPWGER

\begin{tabular}{rllcc} 
Step & Std Error & \multicolumn{3}{l}{ LNINVESTGER } \\
1 & 0.01357873 & 62.094 & 13.773 & 24.132 \\
2 & 0.01977926 & 67.664 & 13.720 & 18.616 \\
3 & 0.02250311 & 69.682 & 15.897 & 14.420 \\
4 & 0.02435643 & 66.440 & 18.100 & 15.460 \\
5 & 0.02636510 & 59.940 & 19.426 & 20.634 \\
6 & 0.02837733 & 53.800 & 20.305 & 25.896 \\
7 & 0.03011886 & 49.389 & 21.227 & 29.384 \\
8 & 0.03151909 & 46.512 & 22.334 & 31.154 \\
9 & 0.03263517 & 44.657 & 23.575 & 31.768
\end{tabular}




$\begin{array}{lllll}10 & 0.03355295 & 43.426 & 24.854 & 31.721 \\ 11 & 0.03434267 & 42.573 & 26.086 & 31.341 \\ 12 & 0.03505048 & 41.953 & 27.220 & 30.826 \\ 13 & 0.03570343 & 41.478 & 28.236 & 30.287 \\ 14 & 0.03631664 & 41.090 & 29.130 & 29.780 \\ 15 & 0.03689853 & 40.753 & 29.911 & 29.336 \\ 16 & 0.03745377 & 40.445 & 30.592 & 28.963 \\ 17 & 0.03798468 & 40.153 & 31.190 & 28.657 \\ 18 & 0.03849214 & 39.873 & 31.718 & 28.409 \\ 19 & 0.03897625 & 39.604 & 32.189 & 28.035 \\ 21 & 0.03987440 & 39.111 & 33.004 & 27.885 \\ 22 & 0.04028908 & 38.889 & 33.361 & 27.750 \\ 23 & 0.04068186 & 38.684 & 33.692 & 27.624 \\ 24 & 0.04105388 & 38.496 & 33.999 & 27.505\end{array}$

compute [vect[strings]] implabel=\| \$

"German Investment",\$

"German GDP",\$

"German Labor Productivity"||

@VARIRF(model=germodel,steps=nsteps,vlabels=implabel,byshocks,byvariables)

* Italy

$*$

Use Clear Program!

calendar(a) 1950

open data

* Penn 7 G7 col a.xls

data(format=xls,org=columns) / popita rgdpchita kiita rgdpwokita

set rgdpita $=$ rgdpchita*popita

set investita $=$ kiita*rgdpita

set lnrgdpita $=\log ($ rgdpita $)$

set lninvestita $=\log ($ investita $)$

set lnlaborpwita $=\log ($ rgdpwokita $)$

graph(key=upleft) 1

\# lnrgdpita

graph(key=upleft) 1

\# lninvestita

graph(key=upleft) 1

\# Inlaborpwita

compute neqn $=3$

compute nlags $=2$

compute nsteps $=12$

grparm(nopatterns) 
grparm(bold) header 36

grparm axislabeling 27

grparm keylabeling 27

system(model=itamodel)

variables lninvestita lnrgdpita lnlaborpwita

lags 1 to nlags

det constant

end(system)

estimate(cvout=vita)

VAR/System - Estimation by Least Squares

Dependent Variable LNINVESTITA

Annual Data From 1952:01 To 2007:01

Usable Observations $56 \quad$ Degrees of Freedom 49

Mean of Dependent Variable 23.804682098

Std Error of Dependent Variable $\quad 0.525349156$

Standard Error of Estimate $\quad 0.071797602$

Sum of Squared Residuals $\quad 0.2525898890$

Durbin-Watson Statistic $\quad 1.960000$

$\begin{array}{lcccc}\text { Variable } & \text { Coeff } & \text { Std Error } & \text { T-Stat } & \text { Signif } \\ \text { ********************************************************************************} \\ \text { 1. LNINVESTITA }\{1\} & 0.619981953 & 0.302981986 & 2.04627 & 0.04611537 \\ \text { 2. LNINVESTITA }\{2\} & 0.155383960 & 0.290548191 & 0.53480 & 0.59520968 \\ \text { 3. LNRGDPITA }\{1\} & 0.792559242 & 1.692470338 & 0.46829 & 0.64165694 \\ \text { 4. LNRGDPITA }\{2\} & -0.482671031 & 1.565872866 & -0.30824 & 0.75920323 \\ \text { 5. LNLABORPWITA }\{1\} & -0.086391311 & 1.056867199 & -0.08174 & 0.93518421 \\ \text { 6. LNLABORPWITA }\{2\} & -0.044773432 & 1.021630812 & -0.04383 & 0.96522165 \\ \text { 7. Constant } & 0.364601900 & 2.860328546 & 0.12747 & 0.89909126\end{array}$

F-Tests, Dependent Variable LNINVESTITA

Variable F-Statistic Signif

LNINVESTITA $\quad 24.2798 \quad 0.0000000$

$\begin{array}{lll}\text { LNRGDPITA } & 0.6195 & 0.5423637\end{array}$

$\begin{array}{lll}\text { LNLABORPWITA } & 0.1202 & 0.8869958\end{array}$

Dependent Variable LNRGDPITA

Annual Data From 1952:01 To 2007:01

Usable Observations $56 \quad$ Degrees of Freedom 49

Mean of Dependent Variable $\quad 20.636568842$

Std Error of Dependent Variable $\quad 0.554543599$

Standard Error of Estimate $\quad 0.017931210$

Sum of Squared Residuals $\quad 0.0157548870$

Durbin-Watson Statistic $\quad 1.922308$

Variable Coeff Std Error T-Stat Signif

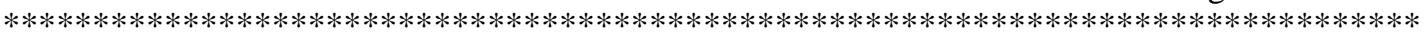

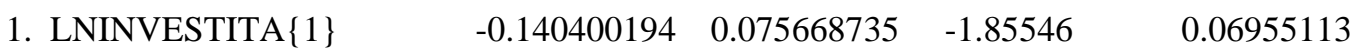

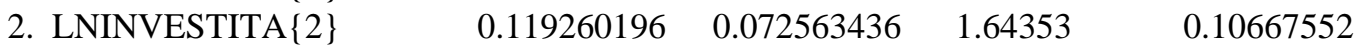

$\begin{array}{lllll}\text { 3. LNRGDPITA }\{1\} & 1.393483939 & 0.422688791 & 3.29671 & 0.00182466\end{array}$

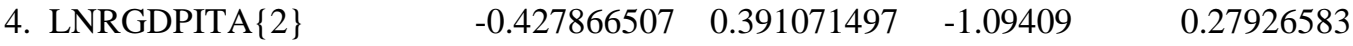

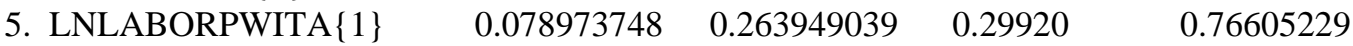

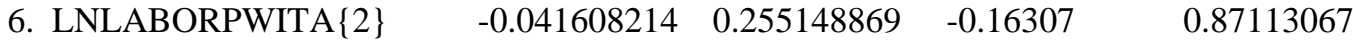


$\begin{array}{lllll}\text { 7. Constant } & 0.838182260 & 0.714357463 & 1.17334 & 0.24633264\end{array}$

F-Tests, Dependent Variable LNRGDPITA

Variable $\quad$ F-Statistic Signif

LNINVESTITA $\quad 1.7471 \quad 0.1849580$

LNRGDPITA $\quad 105.4098 \quad 0.0000000$

LNLABORPWITA $\quad 0.1682 \quad 0.8456561$

Dependent Variable LNLABORPWITA

Annual Data From 1952:01 To 2007:01

Usable Observations $56 \quad$ Degrees of Freedom 49

Mean of Dependent Variable $\quad 10.593339872$

Std Error of Dependent Variable $\quad 0.532236998$

Standard Error of Estimate $\quad 0.018422737$

Sum of Squared Residuals $\quad 0.0166304647$

Durbin-Watson Statistic $\quad 2.047926$

\begin{tabular}{|c|c|c|c|c|}
\hline Variable & Coeff & Std Error & T-Stat & Signif \\
\hline 1. LNINVESTITA $\{1\}$ & -0.165700018 & 0.077742950 & -2.13138 & 0.03809868 \\
\hline 2. LNINVESTITA $\{2\}$ & 0.176709637 & 0.074552530 & 2.37027 & 0.02175497 \\
\hline 3. LNRGDPITA $\{1\}$ & 0.261235020 & 0.434275448 & 0.60154 & 0.55025201 \\
\hline 4. LNRGDPITA $\{2\}$ & -0.255197762 & 0.401791468 & -0.63515 & 0.52828572 \\
\hline 5. LNLABORPWITA $\{1\}$ & 1.306402769 & 0.271184355 & 4.81740 & 0.00001443 \\
\hline 6. LNLABORPWITA $\{2\}$ & -0.345266712 & 0.262142957 & -1.31709 & 0.19393373 \\
\hline 7. Constant & 0.043160673 & 0.733939280 & 0.05881 & 0.95334511 \\
\hline
\end{tabular}

F-Tests, Dependent Variable LNLABORPWITA

Variable $\quad$ F-Statistic Signif

LNINVESTITA $\quad 2.8297 \quad 0.0687107$

$\begin{array}{lll}\text { LNRGDPITA } & 0.2163 & 0.8062714\end{array}$

LNLABORPWITA $98.0495 \quad 0.0000000$

errors(model=itamodel,steps $=24, \mathrm{cv}=\mathrm{vita}$ )

Decomposition of Variance for Series LNINVESTITA

\begin{tabular}{rllll} 
Step & Std Error & \multicolumn{2}{l}{ LNINVESTITA } & \multicolumn{2}{l}{ LNRGDPTA } & LNLABORPWITA \\
1 & 0.06716051 & 100.000 & 0.000 & 0.000 \\
2 & 0.08537212 & 99.623 & 0.370 & 0.007 \\
3 & 0.09640475 & 99.177 & 0.802 & 0.021 \\
4 & 0.10376853 & 98.736 & 1.227 & 0.037 \\
5 & 0.10893871 & 98.308 & 1.642 & 0.050 \\
6 & 0.11269723 & 97.887 & 2.054 & 0.060 \\
7 & 0.11551358 & 97.470 & 2.466 & 0.064 \\
8 & 0.11768796 & 97.056 & 2.881 & 0.064 \\
9 & 0.11941905 & 96.642 & 3.296 & 0.062 \\
10 & 0.12084094 & 96.230 & 3.709 & 0.061 \\
11 & 0.12204530 & 95.819 & 4.118 & 0.064 \\
12 & 0.12309525 & 95.411 & 4.518 & 0.071 \\
13 & 0.12403450 & 95.007 & 4.906 & 0.087 \\
14 & 0.12489338 & 94.610 & 5.281 & 0.110 \\
15 & 0.12569291 & 94.220 & 5.638 & 0.142 \\
16 & 0.12644760 & 93.840 & 6.298 & 0.230 \\
18 & 0.12785873 & 93.119 & 6.597 & 0.285 \\
19 & 0.12852603 & 92.780 & 6.875 & 0.345
\end{tabular}




$\begin{array}{lllll}20 & 0.12917181 & 92.457 & 7.133 & 0.410 \\ 21 & 0.12979745 & 92.152 & 7.371 & 0.477 \\ 22 & 0.13040353 & 91.864 & 7.589 & 0.547 \\ 23 & 0.13099006 & 91.595 & 7.789 & 0.616 \\ 24 & 0.13155674 & 91.344 & 7.971 & 0.686\end{array}$

Decomposition of Variance for Series LNRGDPITA

$\begin{array}{rcccc}\text { Step } & \text { Std Error } & \text { LNINVESTITA } & \text { LNRGDPITA } & \text { LNLABORPWITA } \\ 1 & 0.01677311 & 80.074 & 19.926 & 0.000 \\ 2 & 0.02368397 & 67.938 & 31.990 & 0.072 \\ 3 & 0.02919648 & 61.112 & 38.584 & 0.304 \\ 4 & 0.03390435 & 57.056 & 42.248 & 0.696 \\ 5 & 0.03809535 & 54.548 & 44.241 & 1.211 \\ 6 & 0.04193037 & 52.985 & 45.211 & 1.803 \\ 7 & 0.04550318 & 52.047 & 45.522 & 2.431 \\ 8 & 0.04886910 & 51.545 & 45.395 & 3.060 \\ 9 & 0.05206079 & 51.357 & 44.977 & 3.667 \\ 10 & 0.05509730 & 51.397 & 44.369 & 4.233 \\ 11 & 0.05798951 & 51.605 & 43.645 & 4.751 \\ 12 & 0.06074351 & 51.931 & 42.854 & 5.624 \\ 14 & 0.06584895 & 52.803 & 41.216 & 5.980 \\ 15 & 0.06820396 & 53.298 & 40.414 & 6.288 \\ 16 & 0.07042926 & 53.809 & 39.641 & 6.550 \\ 17 & 0.07252681 & 54.321 & 38.906 & 6.773 \\ 18 & 0.07449910 & 54.826 & 38.215 & 6.960 \\ 19 & 0.07634919 & 55.316 & 37.568 & 7.116 \\ 20 & 0.07808074 & 55.786 & 36.968 & 7.246 \\ 21 & 0.07969789 & 56.234 & 36.414 & 7.353 \\ 22 & 0.08120524 & 56.656 & 35.904 & 7.440 \\ 23 & 0.08260775 & 57.052 & 35.436 & 7.512 \\ 24 & 0.08391059 & 57.422 & 35.008 & 7.570\end{array}$

Decomposition of Variance for Series LNLABORPWITA

\begin{tabular}{rllll} 
Step & Std Error & \multicolumn{3}{l}{ LNINVESTITA LNRGDPITA } \\
1 & 0.01723289 & 53.604 & 24.557 & 21.839 \\
2 & 0.02579972 & 36.841 & 36.786 & 26.373 \\
3 & 0.03323293 & 31.940 & 40.716 & 27.345 \\
4 & 0.03983139 & 33.146 & 40.327 & 26.526 \\
6 & 0.05129330 & 35.452 & 38.855 & 25.693 \\
7 & 0.05634633 & 37.977 & 37.228 & 24.795 \\
8 & 0.06100614 & 40.463 & 35.637 & 23.900 \\
9 & 0.06529653 & 42.794 & 34.163 & 23.043 \\
10 & 0.06923646 & 44.921 & 32.838 & 22.241 \\
11 & 0.07284381 & 46.831 & 31.666 & 21.503 \\
12 & 0.07613689 & 48.530 & 30.639 & 20.830 \\
13 & 0.07913477 & 50.033 & 29.746 & 20.221 \\
14 & 0.08185720 & 51.356 & 28.973 & 19.671 \\
15 & 0.08432427 & 52.518 & 28.304 & 19.178 \\
16 & 0.08655606 & 53.536 & 27.728 & 18.736 \\
17 & 0.08857227 & 54.428 & 27.232 & 18.340 \\
18 & 0.09039191 & 55.208 & 26.805 & 17.986 \\
19 & 0.09203310 & 55.890 & 26.439 & 17.671 \\
20 & 0.09351293 & 56.487 & 26.125 & 17.389 \\
21 & 0.09484728 & 57.008 & 25.855 & 17.138 \\
22 & 0.09605084 & 57.464 & 25.623 & 16.913
\end{tabular}


compute [vect[strings]] implabel=\| \$

"Italian Investment",\$

"Italian GDP",\$

"Italian Labor Productivity"||

@VARIRF(model=itamodel,steps=nsteps,vlabels=implabel,byshocks,byvariables)

* Japan

Use Clear program!

calendar(a) 1950

open data

* Penn 7 G7 col a.xls

data(format=xls,org=columns) / popjpn rgdpchjpn kijpn rgdpwokjpn

set rgdpjpn = rgdpchjpn*popjpn

set investjpn $=$ kijpn ${ }^{*}$ rgdpjpn

set lnrgdpjpn = log(rgdpjpn)

set lninvestjpn $=\log ($ investjpn $)$

set lnlaborpwjpn $=\log ($ rgdpwokjpn $)$

graph(key=upleft) 1

\# lnrgdpjpn

graph(key=upleft) 1

\# lninvestjpn

graph(key=upleft) 1

\# Inlaborpwjpn

compute neqn $=3$

compute nlags $=2$

compute nsteps $=12$

grparm(nopatterns)

grparm(bold) header 36

grparm axislabeling 27

grparm keylabeling 27

system(model=jpnmodel)

variables lninvestjpn lnrgdpjpn lnlaborpwjpn

lags 1 to nlags

det constant

end(system)

estimate(cvout=vjpn) 
VAR/System - Estimation by Least Squares

Dependent Variable LNINVESTJPN

Annual Data From 1952:01 To 2007:01

Usable Observations $56 \quad$ Degrees of Freedom 49

Mean of Dependent Variable $\quad 24.580134688$

Std Error of Dependent Variable $\quad 1.001811580$

Standard Error of Estimate $\quad 0.076950480$

Sum of Squared Residuals $\quad 0.2901474458$

Durbin-Watson Statistic $\quad 1.326403$

\begin{tabular}{|c|c|c|c|c|}
\hline Variable & Coeff & Std Error & T-Stat & Signif \\
\hline & & & & \\
\hline 1. LNINVESTJPN $\{1\}$ & 0.819746122 & 0.177118261 & 4.62824 & 0.00002732 \\
\hline 2. LNINVESTJPN $\{2\}$ & 0.140357430 & 0.163536112 & 0.85827 & 0.39492471 \\
\hline 3. LNRGDPJPN $\{1\}$ & -0.654223903 & 1.665627335 & -0.39278 & 0.69618607 \\
\hline 4. LNRGDPJPN $\{2\}$ & 0.793977649 & 1.594393433 & 0.49798 & 0.62072516 \\
\hline 5. LNLABORPWJPN $\{1\}$ & 2.116745114 & 1.641821230 & 1.28927 & 0.20335845 \\
\hline 6. LNLABORPWJPN $\{2\}$ & -2.257414894 & 1.571921598 & -1.43609 & 0.15733169 \\
\hline 7. Constant & -0.525269507 & 2.933948968 & -0.17903 & 0.85865114 \\
\hline
\end{tabular}

F-Tests, Dependent Variable LNINVESTJPN

$\begin{array}{lll}\text { Variable } & \text { F-Statistic } & \text { Signif } \\ \text { LNINVESTJPN } & 40.7268 & 0.0000000 \\ \text { LNRGDPJPN } & 0.2774 & 0.7589267 \\ \text { LNLABORPWJPN } & 1.0902 & 0.3441697\end{array}$

Dependent Variable LNRGDPJPN

Annual Data From 1952:01 To 2007:01

Usable Observations $56 \quad$ Degrees of Freedom 49

Mean of Dependent Variable $\quad 21.260944834$

Std Error of Dependent Variable $\quad 0.815980171$

Standard Error of Estimate $\quad 0.022551638$

Sum of Squared Residuals $\quad 0.0249202435$

Durbin-Watson Statistic $\quad 1.802708$

$\begin{array}{lllrc}\text { Variable } & \text { Coeff } & \text { Std Error } & \text { T-Stat } & \text { Signif } \\ * * * * * * * * * * * * * * * * * * * * * * * * * * * * * * * * * * * * * * * * * * * * * * * * * * * * * * * * * * * * * * * * * * * * * * * * * * * * * * * \\ \text { 1. LNINVESTJPN }\{1\} & 0.062750290 & 0.051907499 & 1.20889 & 0.23250752 \\ \text { 2. LNINVESTJPN }\{2\} & -0.029411743 & 0.047927021 & -0.61368 & 0.54226690 \\ \text { 3. LNRGDPJPN }\{1\} & 0.291099127 & 0.488140233 & 0.59634 & 0.55369111 \\ \text { 4. LNRGDPJPN }\{2\} & 0.652522740 & 0.467263935 & 1.39648 & 0.16886530 \\ \text { 5. LNLABORPWJPN }\{1\} & 1.066894484 & 0.481163452 & 2.21732 & 0.03127084 \\ \text { 6. LNLABORPWJPN }\{2\} & -1.075265988 & 0.460678184 & -2.33409 & 0.02373518 \\ \text { 7. Constant } & 0.500628868 & 0.859843318 & 0.58223 & 0.56307953\end{array}$

F-Tests, Dependent Variable LNRGDPJPN

Variable $\quad$ F-Statistic Signif

$\begin{array}{lll}\text { LNINVESTJPN } & 0.9160 & 0.4068475\end{array}$

$\begin{array}{lll}\text { LNRGDPJPN } & 63.7544 & 0.0000000\end{array}$

$\begin{array}{lll}\text { LNLABORPWJPN } & 2.7299 & 0.0751521\end{array}$

Dependent Variable LNLABORPWJPN

Annual Data From 1952:01 To 2007:01

Usable Observations $56 \quad$ Degrees of Freedom 49

Mean of Dependent Variable $\quad 10.337415266$ 
Std Error of Dependent Variable

Standard Error of Estimate

Sum of Squared Residuals

Durbin-Watson Statistic
0.655775166
0.021373702
0.0223849223
1.862298

$\begin{array}{lllrl}\text { Variable } & \text { Coeff } & \text { Std Error } & \text { T-Stat } & \text { Signif } \\ * * * * * * * * * * * * * * * * * * * * * * * * * * * * * * * * * * * * * * * * * * * * * * * * * * * * * * * * * * * * * * * * * * * * * * * * * * * * * * * & 1.45365 & 0.15241851 \\ \text { 1. LNINVESTJPN }\{1\} & 0.071514106 & 0.049196223 & 1.456 \\ \text { 2. LNINVESTJPN }\{2\} & -0.025212282 & 0.045423656 & -0.55505 & 0.58138736 \\ \text { 3. LNRGDPJPN }\{1\} & -0.990358774 & 0.462643281 & -2.14065 & 0.03730404 \\ \text { 4. LNRGDPJPN }\{2\} & 0.985192161 & 0.442857411 & 2.22463 & 0.03074377 \\ \text { 5. LNLABORPWJPN }\{1\} & 2.270416175 & 0.456030917 & 4.97865 & 0.00000832 \\ \text { 6. LNLABORPWJPN }\{2\} & -1.359586640 & 0.436615653 & -3.11392 & 0.00308082 \\ \text { 7. Constant } & -0.075603718 & 0.814931256 & -0.09277 & 0.92646209\end{array}$

F-Tests, Dependent Variable LNLABORPWJPN

$\begin{array}{lll}\text { Variable } & \text { F-Statistic } & \text { Signif } \\ \text { LNINVESTJPN } & 1.5983 & 0.2126009 \\ \text { LNRGDPJPN } & 2.5137 & 0.0913580 \\ \text { LNLABORPWJPN } & 31.5767 & 0.0000000\end{array}$

errors(model=jpnmodel,steps=24,cv=vjpn)

Decomposition of Variance for Series LNINVESTJPN

\begin{tabular}{rllll} 
Step & Std Error & \multicolumn{2}{l}{ LNINVESTJPN } & \multicolumn{2}{l}{ LNRGDJPN LNLABORPWJPN } \\
1 & 0.07198058 & 100.000 & 0.000 & 0.000 \\
2 & 0.10858194 & 95.809 & 2.951 & 1.241 \\
3 & 0.14033581 & 93.605 & 3.521 & 2.874 \\
4 & 0.16680919 & 92.641 & 3.536 & 3.822 \\
5 & 0.18971512 & 92.588 & 3.323 & 4.088 \\
6 & 0.21007019 & 92.976 & 3.072 & 3.952 \\
7 & 0.22852797 & 93.522 & 2.835 & 3.643 \\
8 & 0.24542433 & 94.082 & 2.626 & 3.292 \\
9 & 0.26093521 & 94.595 & 2.446 & 2.959 \\
10 & 0.27516425 & 95.037 & 2.292 & 2.671 \\
11 & 0.28818830 & 95.405 & 2.160 & 2.435 \\
12 & 0.30007512 & 95.702 & 2.047 & 2.252 \\
13 & 0.31088945 & 95.934 & 1.950 & 2.116 \\
14 & 0.32069482 & 96.108 & 1.867 & 2.025 \\
15 & 0.32955396 & 96.231 & 1.797 & 1.972 \\
16 & 0.33752900 & 96.311 & 1.737 & 1.953 \\
17 & 0.34468134 & 96.353 & 1.685 & 1.961 \\
18 & 0.35107149 & 96.365 & 1.642 & 1.993 \\
19 & 0.35675879 & 96.351 & 1.606 & 2.044 \\
20 & 0.36180107 & 96.316 & 1.575 & 2.109 \\
21 & 0.36625426 & 96.264 & 1.549 & 2.187 \\
22 & 0.37017210 & 96.199 & 1.528 & 2.273 \\
23 & 0.37360583 & 96.125 & 1.511 & 2.364 \\
24 & 0.37660397 & 96.043 & 1.498 & 2.459
\end{tabular}

Decomposition of Variance for Series LNRGDPJPN

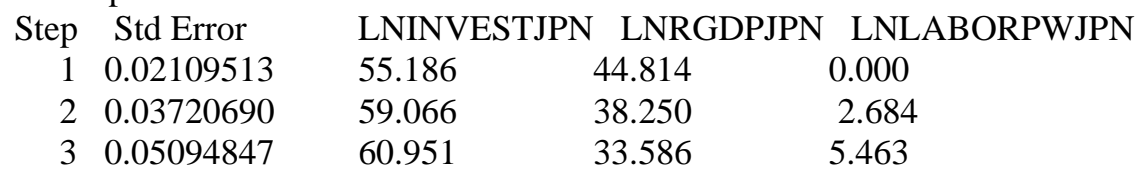




$\begin{array}{rrrrr}4 & 0.06280460 & 63.353 & 29.667 & 6.979 \\ 5 & 0.07337134 & 66.183 & 26.415 & 7.401 \\ 6 & 0.08312073 & 69.137 & 23.685 & 7.178 \\ 7 & 0.09232424 & 71.969 & 21.377 & 6.654 \\ 8 & 0.10110520 & 74.551 & 19.417 & 6.033 \\ 9 & 0.10950539 & 76.838 & 17.748 & 5.414 \\ 10 & 0.11753134 & 78.834 & 16.323 & 4.843 \\ 11 & 0.12517806 & 80.559 & 15.104 & 4.337 \\ 12 & 0.13243889 & 82.042 & 14.057 & 3.901 \\ 13 & 0.13930892 & 83.313 & 13.156 & 3.531 \\ 14 & 0.14578598 & 84.397 & 12.378 & 3.225 \\ 15 & 0.15187077 & 85.321 & 11.705 & 2.974 \\ 16 & 0.15756687 & 86.104 & 11.121 & 2.775 \\ 17 & 0.16288057 & 86.766 & 10.614 & 2.619 \\ 18 & 0.16782063 & 87.324 & 10.173 & 2.503 \\ 19 & 0.17239798 & 87.791 & 9.789 & 2.420 \\ 20 & 0.17662547 & 88.180 & 9.454 & 2.366 \\ 21 & 0.18051745 & 88.502 & 9.161 & 2.337 \\ 22 & 0.18408952 & 88.766 & 8.906 & 2.328 \\ 23 & 0.18735820 & 88.979 & 8.684 & 2.337 \\ 24 & 0.19034058 & 89.150 & 8.489 & 2.360\end{array}$

Decomposition of Variance for Series LNLABORPWJPN

\begin{tabular}{|c|c|c|c|c|}
\hline Step & Std Error & LNINVESTJPN & J LNRGDPJPN & LNLABORPWJPN \\
\hline 1 & 0.01999327 & 48.401 & $43.432 \varepsilon$ & 8.167 \\
\hline 2 & 0.03565881 & 50.589 & 33.610 & 15.802 \\
\hline 3 & 0.04897058 & 52.405 & 27.847 & 19.748 \\
\hline 4 & 0.06036176 & 55.480 & 23.695 & 20.825 \\
\hline 5 & 0.07046987 & 59.267 & 20.550 & 20.183 \\
\hline 6 & 0.07979554 & 63.235 & 18.055 & 18.709 \\
\hline 7 & 0.08861344 & 67.024 & 16.025 & 16.950 \\
\hline 8 & 0.09703829 & 70.455 & 14.349 & 15.196 \\
\hline 9 & 0.10510107 & 73.469 & 12.953 & 13.577 \\
\hline 10 & 0.11279906 & 76.074 & 11.784 & 12.142 \\
\hline 11 & 0.12012014 & 78.304 & 10.800 & 10.896 \\
\hline 12 & 0.12705244 & 80.202 & 9.968 & 9.831 \\
\hline 13 & 0.13358738 & 81.810 & 9.262 & 8.928 \\
\hline 14 & 0.13972044 & 83.169 & 8.660 & 8.171 \\
\hline 15 & 0.14545116 & 84.314 & 8.146 & 7.540 \\
\hline 16 & 0.15078292 & 85.275 & 7.706 & 6.592 \\
\hline 18 & 0.16028010 & 86.750 & 7.003 & 6.248 \\
\hline 19 & 0.16446811 & 87.305 & 6.723 & 5.973 \\
\hline 20 & 0.16830144 & 87.762 & 6.481 & 5.757 \\
\hline 21 & 0.17179665 & 88.135 & 6.272 & 5.592 \\
\hline 22 & 0.17497162 & 88.437 & 6.093 & 5.470 \\
\hline 23 & 0.17784515 & 88.679 & 5.938 & 5.383 \\
\hline 24 & 0.18043659 & 88.869 & 5.804 & 5.327 \\
\hline \multicolumn{5}{|c|}{ compute [vect[strings]] implabel=\| \$ } \\
\hline \multicolumn{5}{|c|}{ "Japanese Investment",\$ } \\
\hline \multicolumn{5}{|c|}{ "Japanese GDP",\$ } \\
\hline \multicolumn{5}{|c|}{ "Japanese Labor Productivity"|| } \\
\hline
\end{tabular}


* United Kingdom

*

Use Clear Program!

calendar(a) 1950

open data

* Penn 7 G7 col b.xls

data(format=xls,org=columns) / popgbr rgdpchgbr kigbr rgdpwokgbr

set rgdpgbr $=$ rgdpchgbr*popgbr

set investgbr $=$ kigbr*rgdpgbr

set lnrgdpgbr $=\log ($ rgdpgbr $)$

set lninvestgbr $=\log ($ investgbr $)$

set lnlaborpwgbr $=\log ($ rgdpwokgbr $)$

graph(key=upleft) 1

\# lnrgdpgbr

graph(key=upleft) 1

\# lninvestgbr

graph(key=upleft) 1

\# lnlaborpwgbr

compute neqn $=3$

compute nlags $=2$

compute nsteps $=12$

grparm(nopatterns)

grparm(bold) header 36

grparm axislabeling 27

grparm keylabeling 27

system(model=gbrmodel)

variables lninvestgbr lnrgdpgbr lnlaborpwgbr

lags 1 to nlags

det constant

end(system)

estimate(cvout=vgbr)

VAR/System - Estimation by Least Squares

Dependent Variable LNINVESTGBR

Annual Data From 1952:01 To 2007:01

Usable Observations $56 \quad$ Degrees of Freedom 49

Mean of Dependent Variable 23.471763739

Std Error of Dependent Variable $\quad 0.518414615$

Standard Error of Estimate $\quad 0.074396728$

Sum of Squared Residuals $\quad 0.2712087822$

Durbin-Watson Statistic $\quad 1.505093$

Variable Coeff Std Error T-Stat Signif 


$\begin{array}{lllcl}\text { 1. LNINVESTGBR }\{1\} & 0.291608936 & 0.209943419 & 1.38899 & 0.17111723 \\ \text { 2. LNINVESTGBR }\{2\} & 0.471022962 & 0.187634608 & 2.51032 & 0.01541312 \\ \text { 3. LNRGDPGBR }\{1\} & 1.561155299 & 1.717216908 & 0.90912 & 0.36773718 \\ \text { 4. LNRGDPGBR }\{2\} & -1.367234036 & 1.649271170 & -0.82899 & 0.41112859 \\ \text { 5. LNLABORPWGBR }\{1\} & 1.936366479 & 1.626939204 & 1.19019 & 0.23970636 \\ \text { 6. LNLABORPWGBR }\{2\} & -1.819204344 & 1.630700804 & -1.11560 & 0.27003620 \\ \text { 7. Constant } & 0.273646444 & 4.897296740 & 0.05588 & 0.95566699\end{array}$

F-Tests, Dependent Variable LNINVESTGBR

Variable F-Statistic Signif

$\begin{array}{lll}\text { LNINVESTGBR } & 30.5318 & 0.0000000\end{array}$

$\begin{array}{lll}\text { LNRGDPGBR } & 0.4150 & 0.6626092\end{array}$

$\begin{array}{lll}\text { LNLABORPWGBR } & 0.7152 & 0.4941243\end{array}$

Dependent Variable LNRGDPGBR

Annual Data From 1952:01 To 2007:01

Usable Observations $56 \quad$ Degrees of Freedom 49

Mean of Dependent Variable 20.802378239

Std Error of Dependent Variable $\quad 0.392521902$

Standard Error of Estimate $\quad 0.015783626$

Sum of Squared Residuals $\quad 0.0122070198$

Durbin-Watson Statistic $\quad 1.818607$

$\begin{array}{lllll}\text { Variable } & \text { Coeff } & \text { Std Error } & \text { T-Stat } & \text { Signif } \\ * * * * * * * * * * * * * * * * * * * * * * * * * * * * * * * * * * * * * * * * * * * * * * * * * * * * * * * * * * * * * * * * * * * * * * * * * * * * * * & \\ \text { 1. LNINVESTGBR }\{1\} & -0.115340423 & 0.044540513 & -2.58956 & 0.01261835 \\ \text { 2. LNINVESTGBR }\{2\} & 0.090991802 & 0.039807591 & 2.28579 & 0.02662989 \\ \text { 3. LNRGDPGBR }\{1\} & 1.324612102 & 0.364315884 & 3.63589 & 0.00066405 \\ \text { 4. LNRGDPGBR }\{2\} & -0.363015500 & 0.349900867 & -1.03748 & 0.30460456 \\ \text { 5. LNLABORPWGBR }\{1\} & 0.415011836 & 0.345163032 & 1.20236 & 0.23500054 \\ \text { 6. LNLABORPWGBR }\{2\} & -0.331383724 & 0.345961074 & -0.95786 & 0.34283372 \\ \text { 7. Constant } & 0.495265653 & 1.038985223 & 0.47668 & 0.63570750\end{array}$

F-Tests, Dependent Variable LNRGDPGBR

Variable F-Statistic Signif

LNINVESTGBR $\quad 3.3538 \quad 0.0431419$

$\begin{array}{lll}\text { LNRGDPGBR } & 28.8753 & 0.0000000\end{array}$

$\begin{array}{lll}\text { LNLABORPWGBR } & 0.7557 & 0.4750642\end{array}$

Dependent Variable LNLABORPWGBR

Annual Data From 1952:01 To 2007:01

Usable Observations $56 \quad$ Degrees of Freedom 49

Mean of Dependent Variable $\quad 10.611052071$

Std Error of Dependent Variable $\quad 0.323833145$

Standard Error of Estimate $\quad 0.015040678$

Sum of Squared Residuals $\quad 0.0110848777$

Durbin-Watson Statistic $\quad 1.947200$

$\begin{array}{lllll}\text { Variable } & \text { Coeff } & \text { Std Error } & \text { T-Stat } & \text { Signif } \\ * * * * * * * * * * * * * * * * * * * * * * * * * * * * * * * * * * * * * * * * * * * * * * * * * * * * * * * * * * * * * * * * * * * * * * * * * & -0.09896956 & 0.02514454 \\ \text { 1. LNINVESTGBR }\{1\} & -0.098043675 & 0.042443955 & -2.30996 & 0.03086586 \\ \text { 2. LNINVESTGBR }\{2\} & 0.084324007 & 0.037933815 & 2.22292 & 0.86738658 \\ \text { 3. LNRGDPGBR }\{1\} & -0.058274493 & 0.347167239 & -0.16786 & 0.71016940 \\ \text { 4. LNRGDPGBR }\{2\} & 0.124634737 & 0.333430748 & 0.37379 & 0.00001109 \\ \text { 5. LNLABORPWGBR }\{1\} & 1.609983669 & 0.328915927 & 4.89482 & \end{array}$




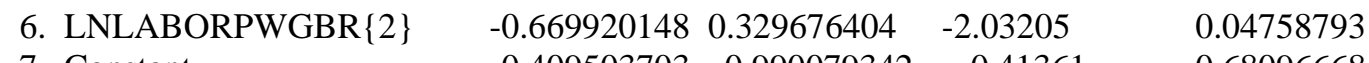

$\begin{array}{lllll}\text { 7. Constant } & -0.409503703 & 0.990079342 & -0.41361 & 0.68096668\end{array}$

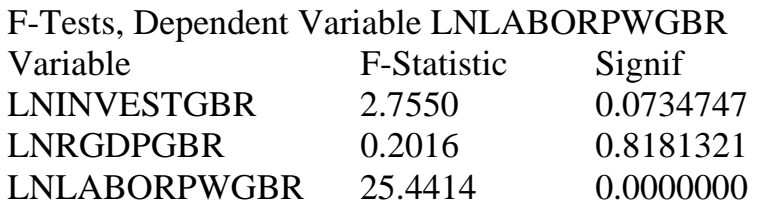

errors(model=gbrmodel,steps=24,cv=vgbr)

Decomposition of Variance for Series LNINVESTGBR

\begin{tabular}{rllrc} 
Step & Std Error & \multicolumn{3}{l}{ LNINVESTGBR LNRGDPGBR LNLABORPWGBR } \\
1 & 0.06959177 & 100.000 & 0.000 & 0.000 \\
2 & 0.09606174 & 89.069 & 9.808 & 1.123 \\
3 & 0.11343320 & 83.828 & 14.013 & 2.159 \\
4 & 0.12537162 & 80.830 & 16.311 & 2.858 \\
5 & 0.13416375 & 78.900 & 17.841 & 3.259 \\
6 & 0.14103392 & 77.449 & 19.082 & 3.469 \\
7 & 0.14663326 & 76.226 & 20.201 & 3.574 \\
8 & 0.15132928 & 75.124 & 21.253 & 3.623 \\
9 & 0.15534956 & 73.133 & 23.207 & 3.660 \\
11 & 0.16193920 & 72.215 & 24.117 & 3.669 \\
12 & 0.16470603 & 71.339 & 24.983 & 3.678 \\
13 & 0.16721483 & 70.502 & 25.809 & 3.689 \\
14 & 0.16951676 & 69.701 & 26.596 & 3.703 \\
15 & 0.17165194 & 68.931 & 27.349 & 3.720 \\
16 & 0.17365196 & 68.191 & 28.069 & 3.740 \\
17 & 0.17554186 & 67.479 & 28.759 & 3.762 \\
18 & 0.17734151 & 66.791 & 29.421 & 3.787 \\
19 & 0.17906679 & 66.127 & 30.058 & 3.814 \\
20 & 0.18073042 & 65.485 & 30.672 & 3.843 \\
21 & 0.18234264 & 64.864 & 31.264 & 3.873 \\
22 & 0.18391173 & 64.261 & 31.835 & 3.904 \\
23 & 0.18544438 & 63.677 & 32.387 & 3.936 \\
24 & 0.18694604 & 63.110 & 32.922 & 3.968
\end{tabular}

Decomposition of Variance for Series LNRGDPGBR

Step Std Error LNINVESTGBR LNRGDPGBR LNLABORPWGBR

$\begin{array}{lllll}1 & 0.01476423 & 61.036 & 38.964 & 0.000\end{array}$

$\begin{array}{lllll}2 & 0.02444169 & 44.396 & 54.807 & 0.797\end{array}$

$\begin{array}{lllll}3 & 0.03157462 & 38.752 & 59.551 & 1.697\end{array}$

$\begin{array}{lllll}4 & 0.03716508 & 35.824 & 61.704 & 2.472\end{array}$

$\begin{array}{lllll}5 & 0.04182353 & 34.048 & 62.871 & 3.081\end{array}$

$\begin{array}{lllll}6 & 0.04590044 & 32.846 & 63.607 & 3.548\end{array}$

$\begin{array}{lllll}7 & 0.04958700 & 31.966 & 64.125 & 3.909\end{array}$

$\begin{array}{lllll}8 & 0.05299016 & 31.288 & 64.517 & 4.195\end{array}$

$\begin{array}{lllll}9 & 0.05617364 & 30.744 & 64.828 & 4.428\end{array}$

$\begin{array}{lllll}10 & 0.05917875 & 30.296 & 65.082 & 4.622\end{array}$

$\begin{array}{lllll}11 & 0.06203448 & 29.920 & 65.295 & 4.785\end{array}$

$\begin{array}{lllll}12 & 0.06476246 & 29.599 & 65.474 & 4.926\end{array}$

$\begin{array}{lllll}13 & 0.06737950 & 29.323 & 65.629 & 5.048\end{array}$

$\begin{array}{lllll}14 & 0.06989902 & 29.082 & 65.763 & 5.155\end{array}$

$\begin{array}{lllll}15 & 0.07233197 & 28.870 & 65.880 & 5.250\end{array}$

$\begin{array}{lllll}16 & 0.07468735 & 28.683 & 65.983 & 5.334\end{array}$ 


$\begin{array}{lllll}17 & 0.07697273 & 28.516 & 66.074 & 5.410 \\ 18 & 0.07919448 & 28.367 & 66.156 & 5.478 \\ 19 & 0.08135808 & 28.232 & 66.229 & 5.539 \\ 20 & 0.08346823 & 28.110 & 66.295 & 5.594 \\ 21 & 0.08552903 & 27.999 & 66.356 & 5.645 \\ 22 & 0.08754407 & 27.898 & 66.410 & 5.691 \\ 23 & 0.08951652 & 27.806 & 66.460 & 5.734 \\ 24 & 0.09144921 & 27.721 & 66.506 & 5.773\end{array}$

Decomposition of Variance for Series LNLABORPWGBR

\begin{tabular}{rllcr} 
Step & Std Error & \multicolumn{3}{l}{ LNINVESTGBR LNRGDPGBR LNL } \\
1 & 0.01406927 & 52.842 & 33.196 & 13.961 \\
2 & 0.02250875 & 36.529 & 43.878 & 19.593 \\
3 & 0.02835898 & 32.060 & 45.572 & 22.369 \\
4 & 0.03285750 & 30.492 & 46.279 & 23.229 \\
5 & 0.03663851 & 30.069 & 46.989 & 22.942 \\
6 & 0.04001628 & 30.094 & 47.831 & 22.076 \\
7 & 0.04313178 & 30.259 & 48.754 & 20.987 \\
8 & 0.04604655 & 30.433 & 49.699 & 19.868 \\
9 & 0.04879038 & 30.566 & 50.623 & 18.811 \\
10 & 0.05138200 & 30.645 & 51.505 & 17.851 \\
11 & 0.05383656 & 30.672 & 52.333 & 16.995 \\
12 & 0.05616784 & 30.657 & 53.106 & 16.238 \\
13 & 0.05838861 & 30.606 & 53.824 & 15.570 \\
14 & 0.06051053 & 30.530 & 54.489 & 14.981 \\
15 & 0.06254415 & 30.435 & 55.107 & 14.459 \\
16 & 0.06449888 & 30.326 & 55.679 & 13.996 \\
17 & 0.06638303 & 30.208 & 56.209 & 13.583 \\
18 & 0.06820389 & 30.085 & 56.702 & 13.213 \\
19 & 0.06996785 & 29.960 & 57.160 & 12.880 \\
20 & 0.07168050 & 29.834 & 57.586 & 12.580 \\
21 & 0.07334670 & 29.709 & 57.983 & 12.308 \\
22 & 0.07497070 & 29.587 & 58.353 & 12.060 \\
23 & 0.07655620 & 29.468 & 58.699 & 11.833 \\
24 & 0.07810645 & 29.353 & 59.022 & 11.625
\end{tabular}

compute [vect[strings]] implabel=|| \$

"British Investment",\$

"British GDP",\$

"British Labor Productivity"||

@VARIRF(model=gbrmodel,steps=nsteps,vlabels=implabel,byshocks,byvariables)

* USA

$*$

Use Clear Program!

calendar(a) 1950

open data

* Penn 7 G7 col b.xls 


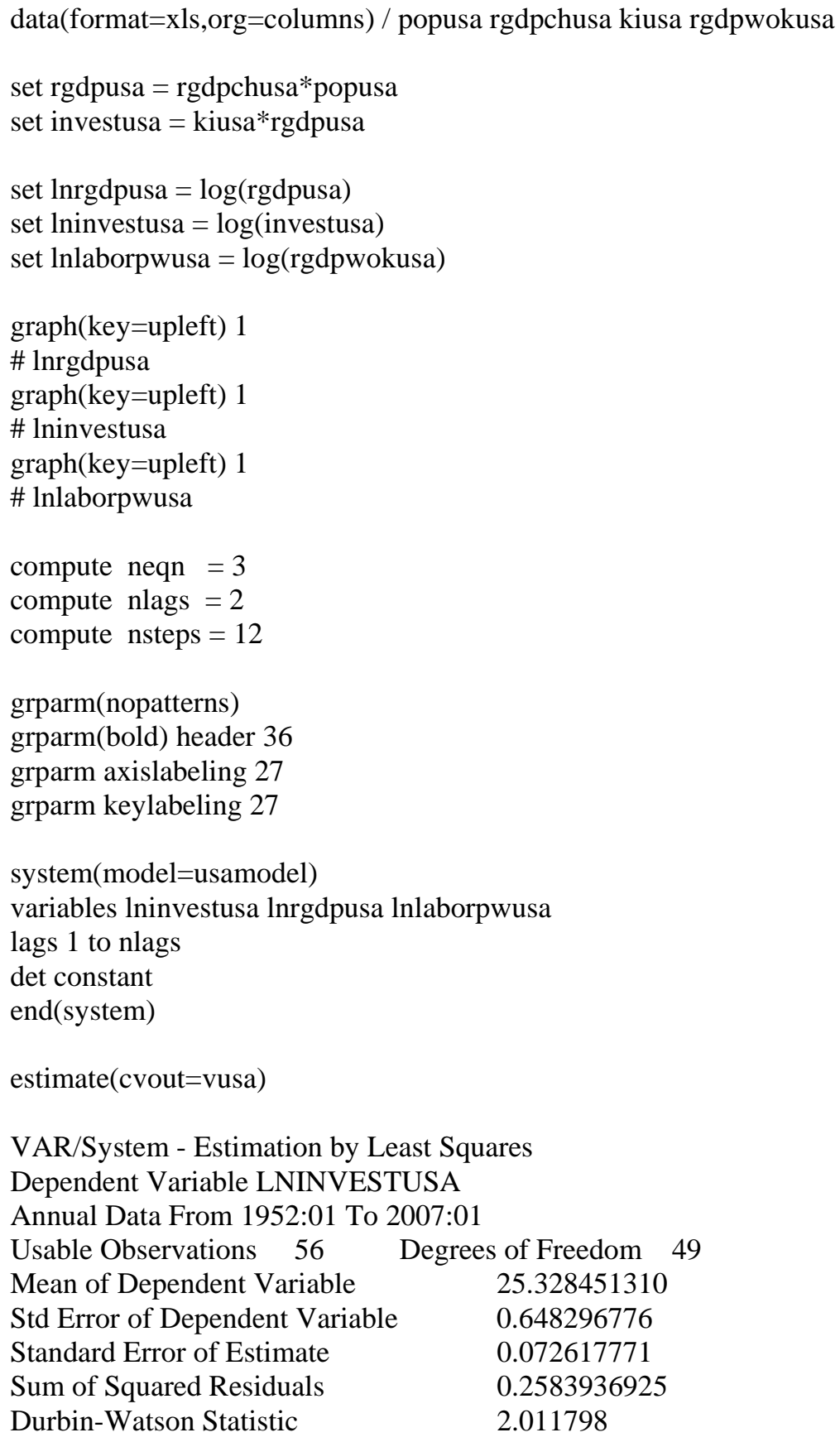

VAR/System - Estimation by Least Squares Dependent Variable LNINVESTUSA Annual Data From 1952:01 To 2007:01

\begin{tabular}{|c|c|c|c|c|}
\hline Variable & Coeff & Std Error & T-Stat & Signif \\
\hline 1. LNINVESTUSA $\{1\}$ & 0.557075064 & 0.288435827 & 1.93137 & 0.05923271 \\
\hline 2. LNINVESTUSA $\{2\}$ & -0.262449025 & 0.208262601 & -1.26018 & 0.21357187 \\
\hline 3. LNRGDPUSA $\{1\}$ & -1.376308459 & 1.839071417 & -0.74837 & 0.45781333 \\
\hline 4. LNRGDPUSA $\{2\}$ & 1.989154880 & 1.760155949 & 1.13010 & 0.26393510 \\
\hline 5. LNLABORPWUSA $\{1\}$ & 2.361346089 & 1.608508389 & 1.46803 & 0.14848515 \\
\hline 6. LNLABORPWUSA $\{2\}$ & -1.854596149 & 1.541146227 & -1.20339 & 0.23460828 \\
\hline 7. Constant & -1.350635572 & 0.632227345 & -2.13631 & 0.03767425 \\
\hline
\end{tabular}

F-Tests, Dependent Variable LNINVESTUSA

Variable F-Statistic Signif 


$\begin{array}{lll}\text { LNINVESTUSA } & 1.8966 & 0.1609296 \\ \text { LNRGDPUSA } & 6.1192 & 0.0042435 \\ \text { LNLABORPWUSA } & 1.7533 & 0.1838848\end{array}$

Dependent Variable LNRGDPUSA

Annual Data From 1952:01 To 2007:01

Usable Observations $56 \quad$ Degrees of Freedom 49

Mean of Dependent Variable $\quad 22.440452300$

Std Error of Dependent Variable $\quad 0.528812885$

Standard Error of Estimate $\quad 0.021894100$

Sum of Squared Residuals $\quad 0.0234882283$

Durbin-Watson Statistic $\quad 2.139537$

$\begin{array}{lcccc}\text { Variable } & \text { Coeff } & \text { Std Error } & \text { T-Stat } & \text { Signif } \\ * * * * * * * * * * * * * * * * * * * * * * * * * * * * * * * * * * * * * * * * * * * * * * * * * * * * * * * * * * * * * * * * * * * * * * * * * * * * * & \\ \text { 1. LNINVESTUSA }\{1\} & -0.099233748 & 0.086962773 & -1.14111 & 0.25937214 \\ \text { 2. LNINVESTUSA }\{2\} & -0.006660139 & 0.062790720 & -0.10607 & 0.91596078 \\ \text { 3. LNRGDPUSA }\{1\} & 0.884162267 & 0.554476023 & 1.59459 & 0.11723444 \\ \text { 4. LNRGDPUSA }\{2\} & 0.203992957 & 0.530683181 & 0.38440 & 0.70234755 \\ \text { 5. LNLABORPWUSA }\{1\} & 0.492675243 & 0.484961772 & 1.01591 & 0.31466462 \\ \text { 6. LNLABORPWUSA }\{2\} & -0.413609865 & 0.464652228 & -0.89015 & 0.37773547 \\ \text { 7. Constant } & -0.126825867 & 0.190615166 & -0.66535 & 0.50894632\end{array}$

F-Tests, Dependent Variable LNRGDPUSA

Variable F-Statistic Signif

LNINVESTUSA $\quad 1.6492 \quad 0.2026862$

$\begin{array}{lll}\text { LNRGDPUSA } & 152.7530 & 0.0000000\end{array}$

$\begin{array}{lll}\text { LNLABORPWUSA } & 0.6512 & 0.5258568\end{array}$

Dependent Variable LNLABORPWUSA

Annual Data From 1952:01 To 2007:01

Usable Observations $56 \quad$ Degrees of Freedom 49

Mean of Dependent Variable $\quad 10.905589641$

Std Error of Dependent Variable $\quad 0.251500632$

Standard Error of Estimate $\quad 0.021324024$

Sum of Squared Residuals $\quad 0.0222809856$

Durbin-Watson Statistic $\quad 2.212136$

$\begin{array}{lcccc}\text { Variable } & \text { Coeff } & \text { Std Error } & \text { T-Stat } & \text { Signif } \\ * * * * * * * * * * * * * * * * * * * * * * * * * * * * * * * * * * * * * * * * * * * * * * * * * * * * * * * * * * * * * * * * * * * * * * * * * * * \\ \text { 1. LNINVESTUSA }\{1\} & -0.078746632 & 0.084698447 & -0.92973 & 0.35706908 \\ \text { 2. LNINVESTUSA }\{2\} & 0.003544767 & 0.061155783 & 0.05796 & 0.95401391 \\ \text { 3. LNRGDPUSA }\{1\} & -0.795197730 & 0.540038645 & -1.47248 & 0.14728507 \\ \text { 4. LNRGDPUSA }\{2\} & 0.868399061 & 0.516865319 & 1.68013 & 0.09929823 \\ \text { 5. LNLABORPWUSA }\{1\} & 2.047271515 & 0.472334398 & 4.33437 & 0.00007245 \\ \text { 6. LNLABORPWUSA }\{2\} & -1.013193506 & 0.452553671 & -2.23884 & 0.02974081 \\ \text { 7. Constant } & -0.081737829 & 0.185651952 & -0.44027 & 0.66167440\end{array}$

F-Tests, Dependent Variable LNLABORPWUSA

$\begin{array}{lll}\text { Variable } & \text { F-Statistic } & \text { Signif } \\ \text { LNINVESTUSA } & 0.8708 & 0.4250092 \\ \text { LNRGDPUSA } & 2.9260 & 0.0630353 \\ \text { LNLABORPWUSA } & 58.8770 & 0.0000000\end{array}$

errors(model=usamodel,steps=24,cv=vusa) 
Decomposition of Variance for Series LNINVESTUSA

\begin{tabular}{rllcc} 
Step & Std Error & \multicolumn{3}{l}{ LNINVESTUSA LNRGDPUSA LNLABORPWUSA } \\
1 & 0.06792770 & 100.000 & 0.000 & 0.000 \\
2 & 0.08719728 & 97.386 & 1.228 & 1.386 \\
3 & 0.09421923 & 91.708 & 4.320 & 3.972 \\
4 & 0.09870795 & 86.126 & 7.710 & 6.165 \\
5 & 0.10255479 & 82.085 & 10.392 & 7.523 \\
6 & 0.10627313 & 79.331 & 12.364 & 8.305 \\
7 & 0.10989212 & 77.299 & 13.917 & 8.784 \\
8 & 0.11328805 & 75.616 & 15.274 & 9.110 \\
9 & 0.11642280 & 74.143 & 16.522 & 9.335 \\
10 & 0.11932930 & 72.845 & 17.680 & 9.475 \\
11 & 0.12205203 & 71.705 & 18.751 & 9.544 \\
12 & 0.12462317 & 70.700 & 19.743 & 9.557 \\
13 & 0.12706288 & 69.804 & 20.668 & 9.527 \\
14 & 0.12938535 & 68.997 & 21.536 & 9.467 \\
15 & 0.13160266 & 68.262 & 22.355 & 9.383 \\
16 & 0.13372575 & 67.586 & 23.131 & 9.282 \\
17 & 0.13576441 & 66.963 & 23.868 & 9.169 \\
18 & 0.13772705 & 66.383 & 24.569 & 9.048 \\
19 & 0.13962088 & 65.842 & 25.237 & 8.921 \\
20 & 0.14145210 & 65.334 & 25.876 & 8.790 \\
21 & 0.14322605 & 64.856 & 26.487 & 8.658 \\
22 & 0.14494744 & 64.404 & 27.071 & 8.525 \\
23 & 0.14662037 & 63.976 & 27.632 & 8.392 \\
24 & 0.14824848 & 63.568 & 28.170 & 8.261
\end{tabular}

Decomposition of Variance for Series LNRGDPUSA

\begin{tabular}{rcccc} 
Step & Std Error & \multicolumn{2}{l}{ LNINVESTUSA LNRGDPUSA LNLABORPWUSA } \\
1 & 0.02048005 & 78.662 & 21.338 & 0.000 \\
2 & 0.03012439 & 70.730 & 28.765 & 0.505 \\
3 & 0.03641530 & 63.079 & 35.672 & 1.250 \\
4 & 0.04131855 & 57.729 & 40.473 & 1.797 \\
5 & 0.04558188 & 54.603 & 43.292 & 2.105 \\
6 & 0.04946862 & 52.881 & 44.852 & 2.267 \\
7 & 0.05304911 & 51.842 & 45.800 & 2.358 \\
8 & 0.05635075 & 51.098 & 46.491 & 2.411 \\
9 & 0.05940871 & 50.502 & 47.061 & 2.437 \\
10 & 0.06226242 & 50.008 & 47.551 & 2.441 \\
11 & 0.06494562 & 49.594 & 47.978 & 2.428 \\
12 & 0.06748359 & 49.244 & 48.354 & 2.401 \\
13 & 0.06989499 & 48.942 & 48.692 & 2.366 \\
14 & 0.07219436 & 48.675 & 49.001 & 2.324 \\
15 & 0.07439374 & 48.436 & 49.287 & 2.277 \\
16 & 0.07650330 & 48.219 & 49.552 & 2.229 \\
17 & 0.07853172 & 48.019 & 49.801 & 2.179 \\
18 & 0.08048639 & 47.836 & 50.036 & 2.129 \\
19 & 0.08237366 & 47.665 & 50.256 & 2.079 \\
20 & 0.08419901 & 47.505 & 50.465 & 2.030 \\
21 & 0.08596722 & 47.356 & 50.663 & 1.982 \\
22 & 0.08768248 & 47.215 & 50.850 & 1.889 \\
24 & 0.09096858 & 46.956 & 51.198 & 1.845
\end{tabular}

Decomposition of Variance for Series LNLABORPWUSA 


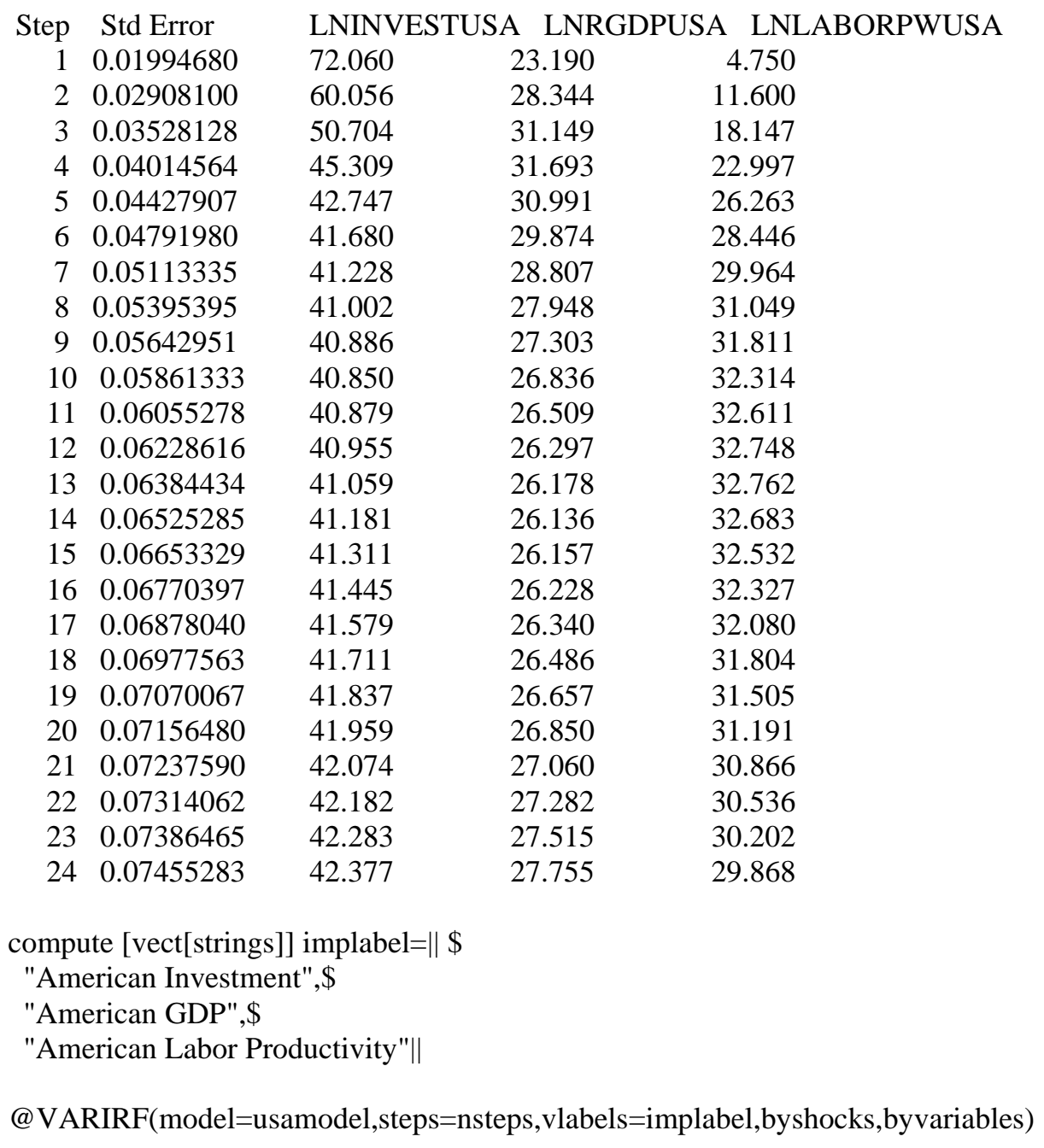




\section{B) Vector Error Correction Models}

This section uses the program ECT.PRG of RATS 7.2 (RATS User’s Guide, Example 10.6).

Variables are nonstationary and cointegrated in Australia, France and Japan. In the other countries the VECMs are inappropriate.

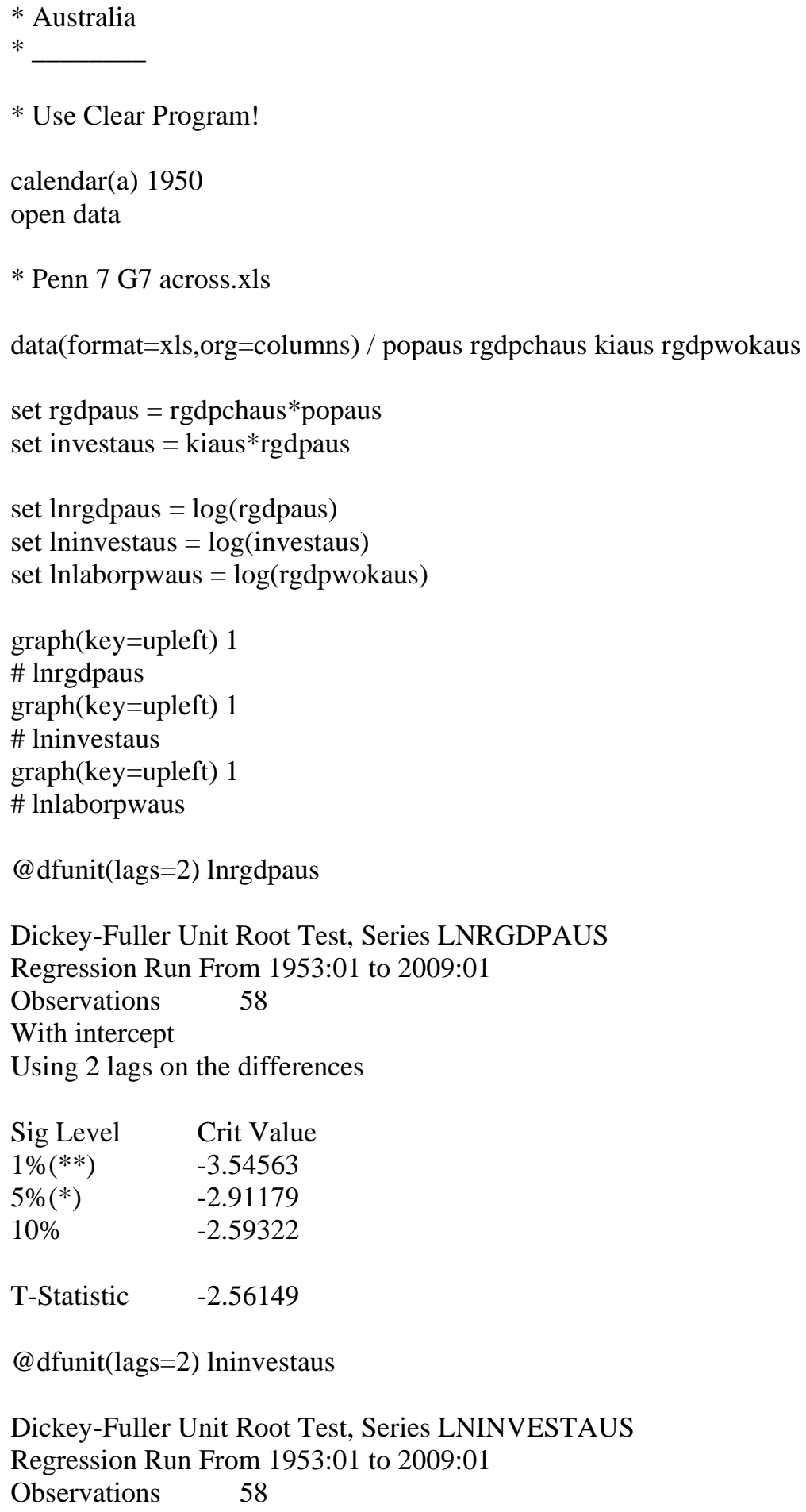


With intercept

Using 2 lags on the differences

$\begin{array}{ll}\text { Sig Level } & \text { Crit Value } \\ 1 \%\left({ }^{*}\right) & -3.54563 \\ 5 \%\left(^{*}\right) & -2.91179 \\ 10 \% & -2.59322\end{array}$

T-Statistic $\quad-0.23326$

@dfunit(lags=2) lnlaborpwaus

Dickey-Fuller Unit Root Test, Series LNLABORPWAUS

Regression Run From 1953:01 to 2007:01

Observations $\quad 56$

With intercept

Using 2 lags on the differences

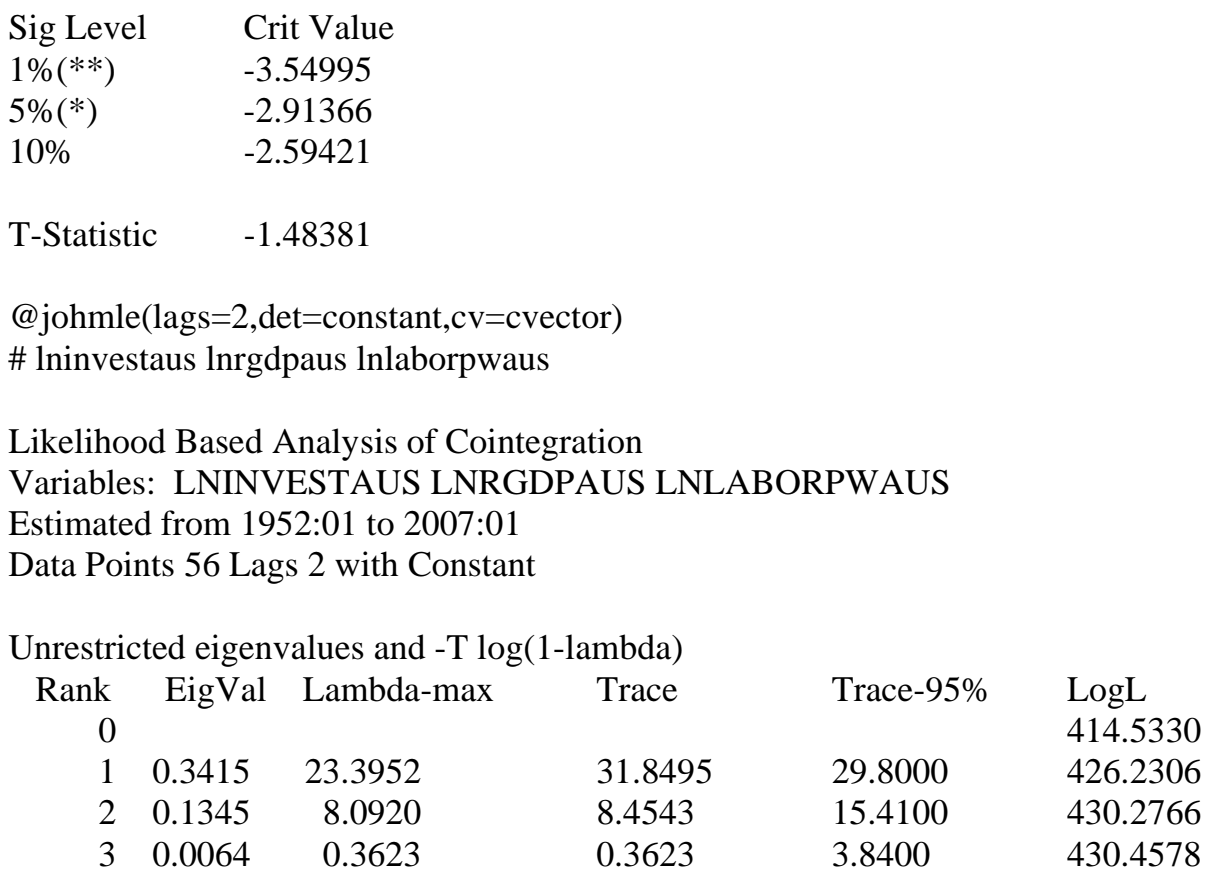

Cointegrating Vector for Largest Eigenvalue

$\begin{array}{lll}\text { LNINVESTAUS } & \text { LNRGDPAUS } & \text { LNLABORPWAUS } \\ -10.930932 & 0.636661 & 25.624356\end{array}$

equation(coeffs=cvector) ecteq *

\# lninvestaus lnrgdpaus lnlaborpwaus

system(model=ectmodel)

variables lninvestaus lnrgdpaus lnlaborpwaus

lags 1 to 2

det constant

ect ecteq

end(system)

estimate 
VAR/System - Estimation by Cointegrated Least Squares

Dependent Variable LNINVESTAUS

Annual Data From 1952:01 To 2007:01

Usable Observations $56 \quad$ Degrees of Freedom 51

Mean of Dependent Variable $\quad 0.0422482047$

Std Error of Dependent Variable $\quad 0.1209219919$

Standard Error of Estimate $\quad 0.1007564406$

Sum of Squared Residuals $\quad 0.5177448766$

Durbin-Watson Statistic $\quad 1.640865$

\begin{tabular}{lllll}
\multicolumn{1}{l}{ Variable } & Coeff & Std Error & T-Stat & Signif \\
$* * * * * * * * * * * * * * * * * * * * * * * * * * * * * * * * * * * * * * * * * * * * * * * * * * * * * * * * * * * * * * * * * * * * * * * * * * * * * * * * *$ & \\
1. D_LNINVESTAUS(1) & -0.869767764 & 0.228295515 & -3.80983 & 0.00037537 \\
2. D_LNRGDPAUS(1) & 2.693711655 & 2.048142139 & 1.31520 & 0.19432626 \\
3. D_LNLABORPWAUS(1) & 1.104770495 & 1.826500991 & 0.60486 & 0.54795642 \\
4. Constant & -1.550447264 & 0.562111778 & -2.75825 & 0.00804728 \\
5. EC1\{1\} & 0.037430470 & 0.013464146 & 2.78001 & 0.00759457
\end{tabular}

Dependent Variable LNRGDPAUS

Annual Data From 1952:01 To 2007:01

Usable Observations $56 \quad$ Degrees of Freedom 51

Mean of Dependent Variable $\quad 0.0386653687$

Std Error of Dependent Variable $\quad 0.0251823872$

Standard Error of Estimate $\quad 0.0220799177$

Sum of Squared Residuals $\quad 0.0248636610$

Durbin-Watson Statistic $\quad 1.892016$

\begin{tabular}{|c|c|c|c|c|}
\hline Variable & Coeff & Std Error & T-Stat & Signif \\
\hline & & 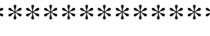 & & \\
\hline 1. D_LNINVESTAUS(1) & -0.217400951 & 0.050029022 & -4.34550 & 0.00006634 \\
\hline 2. D_LNRGDPAUS(1) & 0.633736356 & 0.448832944 & & 0.16403300 \\
\hline 3. D_LNLABORPWAUS(1) & 0.295894810 & 0.400262169 & 0.73925 & 0.46314275 \\
\hline 4. Constant & -0.048614121 & 0.123182019 & -0.39465 & 0.69474483 \\
\hline 5. EC1 $1\{1\}$ & 0.001660215 & 0.002950553 & 0.56268 & 0.57611859 \\
\hline
\end{tabular}

Dependent Variable LNLABORPWAUS

Annual Data From 1952:01 To 2007:01

Usable Observations $56 \quad$ Degrees of Freedom 51

Mean of Dependent Variable $\quad 0.0189670408$

Std Error of Dependent Variable $\quad 0.0261087377$

Standard Error of Estimate $\quad 0.0235427831$

Sum of Squared Residuals $\quad 0.0282673945$

Durbin-Watson Statistic 2.010136

\begin{tabular}{|c|c|c|c|c|}
\hline Variable & Coeff & Std Error & T-Stat & Signif \\
\hline 1 D I NINVESTAUS(1) & & & & \\
\hline 1. D_LNRGDPAUS(1) & $\begin{array}{l}-0.192926122 \\
0.361812981\end{array}$ & $\begin{array}{l}0.053343605 \\
0.478569568\end{array}$ & $\begin{array}{l}-3.61667 \\
075603\end{array}$ & $\begin{array}{l}0.00068392 \\
045310924\end{array}$ \\
\hline 3. D LNLABORPWAUS(1) & 0.400192849 & 0.426780824 & 0.93770 & 0.35281767 \\
\hline 4. Constant & -0.108233670 & 0.131343224 & -0.82405 & 0.41374719 \\
\hline 5. EC1 $1\{1\}$ & 0.002837613 & 0.003146037 & 0.90196 & 0.37131595 \\
\hline
\end{tabular}

compute sigma $=\%$ sigma

errors(model=ectmodel,steps $=24$ ) 
Decomposition of Variance for Series LNINVESTAUS

$\begin{array}{rcccc}\text { Step } & \text { Std Error } & \text { LNINVESTAUS } & \text { LNRGDPAUS } & \text { LNLABORPWAUS } \\ 1 & 0.09615323 & 100.000 & 0.000 & 0.000 \\ 2 & 0.12479676 & 84.857 & 13.570 & 1.574 \\ 3 & 0.14509437 & 80.278 & 17.138 & 2.584 \\ 4 & 0.16211273 & 77.844 & 18.923 & 3.233 \\ 5 & 0.17696010 & 75.929 & 20.309 & 3.761 \\ 6 & 0.19020291 & 74.371 & 21.410 & 4.220 \\ 7 & 0.20223633 & 73.061 & 22.314 & 4.626 \\ 8 & 0.21332638 & 71.927 & 23.081 & 4.992 \\ 9 & 0.22365899 & 70.927 & 23.747 & 5.326 \\ 10 & 0.23337007 & 70.036 & 24.332 & 5.632 \\ 11 & 0.24256190 & 69.233 & 24.853 & 5.913 \\ 12 & 0.25131319 & 68.506 & 25.321 & 6.173 \\ 13 & 0.25968570 & 67.843 & 25.744 & 6.413 \\ 14 & 0.26772872 & 67.237 & 26.127 & 6.636 \\ 15 & 0.27548211 & 66.680 & 26.478 & 6.843 \\ 16 & 0.28297855 & 66.167 & 26.799 & 7.035 \\ 17 & 0.29024511 & 65.693 & 27.094 & 7.213 \\ 18 & 0.29730448 & 65.254 & 27.366 & 7.380 \\ 19 & 0.30417579 & 64.847 & 27.617 & 7.535 \\ 20 & 0.31087538 & 64.469 & 27.851 & 7.680 \\ 21 & 0.31741728 & 64.116 & 28.067 & 7.816 \\ 22 & 0.32381362 & 63.787 & 28.269 & 7.943 \\ 23 & 0.33007500 & 63.480 & 28.458 & 8.062 \\ 24 & 0.33621069 & 63.192 & 28.634 & 8.174\end{array}$

Decomposition of Variance for Series LNRGDPAUS

$\begin{array}{rllcc}\text { Step } & \text { Std Error } & \text { LNINVESTAS } & \text { LNRGDPAUS } & \text { LNLABORPWAUS } \\ 1 & 0.02107116 & 79.189 & 20.811 & 0.000 \\ 2 & 0.03189335 & 54.892 & 44.460 & 0.648 \\ 3 & 0.03988649 & 52.254 & 47.069 & 0.677 \\ 4 & 0.04709592 & 52.680 & 46.744 & 0.576 \\ 5 & 0.05383324 & 53.657 & 45.868 & 0.475 \\ 6 & 0.06019675 & 54.810 & 44.800 & 0.391 \\ 7 & 0.06625550 & 55.971 & 43.704 & 0.325 \\ 8 & 0.07205257 & 57.068 & 42.658 & 0.275 \\ 9 & 0.07761691 & 58.074 & 41.689 & 0.237 \\ 10 & 0.08297011 & 58.985 & 40.806 & 0.209 \\ 11 & 0.08812947 & 59.805 & 40.007 & 0.188 \\ 12 & 0.09310948 & 60.540 & 39.287 & 0.173 \\ 13 & 0.09792280 & 61.200 & 38.638 & 0.161 \\ 14 & 0.10258069 & 61.792 & 38.055 & 0.153 \\ 15 & 0.10709334 & 62.325 & 37.528 & 0.147 \\ 16 & 0.11147007 & 62.804 & 37.053 & 0.143 \\ 17 & 0.11571945 & 63.237 & 36.623 & 0.140 \\ 18 & 0.11984936 & 63.629 & 36.233 & 0.138 \\ 19 & 0.12386710 & 63.984 & 35.879 & 0.136 \\ 20 & 0.12777941 & 64.307 & 35.557 & 0.136 \\ 21 & 0.13159255 & 64.602 & 35.262 & 0.135 \\ 22 & 0.13531230 & 64.872 & 34.993 & 0.135 \\ 23 & 0.13894401 & 65.118 & 34.746 & 0.135 \\ 24 & 0.14249265 & 65.345 & 34.519 & 0.136\end{array}$


Decomposition of Variance for Series LNLABORPWAUS

\begin{tabular}{|c|c|c|c|c|}
\hline Step & Std Error 1 & LNINVESTAUS & LNRGDPAUS & LNLABORPWAUS \\
\hline 1 & 0.02246720 & 70.279 & 18.324 & 11.398 \\
\hline 2 & 0.03333461 & 47.197 & 36.393 & 16.410 \\
\hline 3 & 0.04091259 & 43.952 & 38.124 & 17.924 \\
\hline 4 & 0.04746012 & 43.413 & 38.401 & 18.186 \\
\hline 5 & 0.05338329 & 43.474 & 38.448 & 18.079 \\
\hline 6 & 0.05883507 & 43.828 & 38.338 & 17.834 \\
\hline 7 & 0.06392166 & 44.311 & 38.151 & 17.537 \\
\hline 8 & 0.06871265 & 44.838 & 37.934 & 17.228 \\
\hline 9 & 0.07325569 & 45.368 & 37.708 & 16.924 \\
\hline 10 & 0.07758570 & 45.881 & 37.484 & 16.635 \\
\hline 11 & 0.08172932 & 46.367 & 37.269 & 16.364 \\
\hline 12 & 0.08570761 & 46.822 & 37.065 & 16.113 \\
\hline 13 & 0.08953764 & 47.245 & 36.873 & 15.882 \\
\hline 14 & 0.09323359 & 47.636 & 36.695 & 15.669 \\
\hline 15 & 0.09680742 & 47.997 & 36.529 & 15.474 \\
\hline 16 & 0.10026939 & 48.330 & 36.376 & 15.294 \\
\hline 17 & 0.10362842 & 48.636 & 36.234 & 15.130 \\
\hline 18 & 0.10689230 & 48.919 & 36.103 & 14.978 \\
\hline 19 & 0.11006794 & 49.180 & 35.981 & 14.839 \\
\hline 20 & 0.11316147 & 49.421 & 35.869 & 14.710 \\
\hline 21 & 0.11617839 & 49.643 & 35.765 & 14.592 \\
\hline 22 & 0.11912364 & 49.849 & 35.668 & 14.483 \\
\hline 23 & 0.12200169 & 50.040 & 35.579 & 14.381 \\
\hline 24 & 0.12481658 & 50.217 & 35.495 & 14.287 \\
\hline \multicolumn{5}{|c|}{ compute nsteps = 12} \\
\hline \multicolumn{5}{|c|}{ compute [vect[strings]] implabel=\| \$ } \\
\hline \multicolumn{5}{|c|}{ "Australian Investment",\$ } \\
\hline \multicolumn{5}{|c|}{ "Australian GDP",\$ } \\
\hline \multicolumn{5}{|c|}{ "Australian Labor Productivity"|| } \\
\hline \multicolumn{5}{|c|}{ grparm(nopatterns) } \\
\hline \multicolumn{5}{|c|}{ grparm(bold) header 36} \\
\hline \multicolumn{5}{|c|}{ grparm axislabeling 27} \\
\hline \multicolumn{5}{|c|}{ grparm keylabeling 27} \\
\hline
\end{tabular}

@VARIRF(model=ectmodel,steps=nsteps,vlables=implabel,byshocks,byvariables)

\footnotetext{
* Canada

$*$

* Use Clear Program!

calendar(a) 1950

open data

* Penn 7 G7 across.xls

data(format=xls,org=columns) / popcan rgdpchcan kican rgdpwokcan
} 


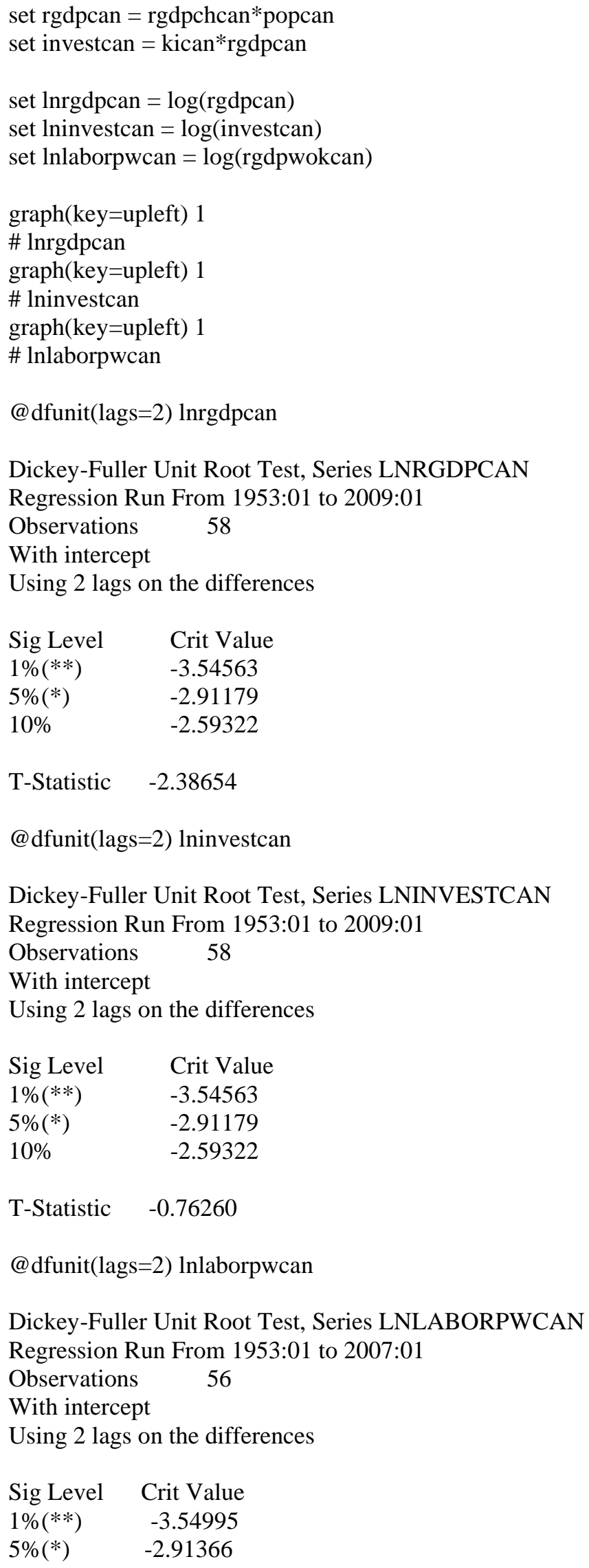




\begin{tabular}{|c|c|c|c|c|c|}
\hline \multicolumn{6}{|c|}{$10 \% \quad-2.59421$} \\
\hline \multicolumn{3}{|c|}{$\begin{array}{l}\text { T-Statistic } \quad-0.69397 \\
\text { @johmle(lags=2,det }=\text { constant, } \mathrm{cv}=\text { cvector })\end{array}$} & & & \\
\hline \multicolumn{6}{|c|}{$\begin{array}{l}\text { Likelihood Based Analysis of Cointegration } \\
\text { Variables: LNINVESTCAN LNRGDPCAN LNLABORPWCAN } \\
\text { Estimated from 1952:01 to 2007:01 } \\
\text { Data Points } 56 \text { Lags } 2 \text { with Constant }\end{array}$} \\
\hline \multicolumn{6}{|c|}{ Unrestricted eigenvalues and -T $\log (1-\operatorname{lambda})$} \\
\hline $\begin{array}{r}\text { Rank } \\
0\end{array}$ & EigVal & Lambda-max & Trace & Trace-95\% & $\begin{array}{l}\text { LogL } \\
425.5518\end{array}$ \\
\hline 1 & 0.2426 & 15.5614 & 27.9067 & 29.8000 & 433.3325 \\
\hline 2 & 0.1493 & 9.0559 & 12.3453 & 15.4100 & 437.8605 \\
\hline 3 & 0.0570 & 3.2894 & 3.2894 & 3.84004 & 39.5052 \\
\hline
\end{tabular}

Cointegrating Vector for Largest Eigenvalue

LNINVESTCAN LNRGDPCAN LNLABORPWCAN

$-10.347156 \quad 0.887710 \quad 25.440965$

equation(coeffs=cvector) ecteq *

\# lninvestcan lnrgdpcan lnlaborpwcan

system(model=ectmodel)

variables lninvestcan Inrgdpcan Inlaborpwcan

lags 1 to 2

det constant

ect ecteq

end(system)

estimate

VAR/System - Estimation by Cointegrated Least Squares Dependent Variable LNINVESTCAN

Annual Data From 1952:01 To 2007:01

Usable Observations $56 \quad$ Degrees of Freedom 51

Mean of Dependent Variable $\quad 0.0418080311$

Std Error of Dependent Variable $\quad 0.0853633925$

Standard Error of Estimate $\quad 0.0816829220$

Sum of Squared Residuals $\quad 0.3402770874$

Durbin-Watson Statistic $\quad 1.974118$

\begin{tabular}{|c|c|c|c|c|}
\hline Variable & Coeff & Std Error & T-Stat & Signif \\
\hline 1 D ININVESTCAN(1) & & & & \\
\hline $\begin{array}{l}\text { 1. D_LNINVESTCAN(1) } \\
\text { 2. D LNRGDPCAN(1) }\end{array}$ & -0.117229009 & 0.242230427 & -0.48396 & 0.63048838 \\
\hline $\begin{array}{l}\text { 2. D_LNRGDPCAN(1) } \\
\text { 3. D LNLABORPWCAN(1) }\end{array}$ & -1.925014937 & 1.534892252 & -1.25417 & 0.21549848 \\
\hline 3. D_LNLABORPWCAN(1) & 2.461865575 & 1.203881272 & 2.04494 & 0.04603759 \\
\hline 4. Constant & -1.049712467 & 0.574903245 & -1.82589 & 0.07372339 \\
\hline 5. EC1 11$\}$ & 0.020652181 & 0.010915340 & 1.89203 & 0.06417042 \\
\hline
\end{tabular}

Dependent Variable LNRGDPCAN

Annual Data From 1952:01 To 2007:01

Usable Observations $56 \quad$ Degrees of Freedom 51 
Mean of Dependent Variable

Std Error of Dependent Variable

Standard Error of Estimate

Sum of Squared Residuals

Durbin-Watson Statistic
0.0356179097
0.0220667157
0.0189155736
0.0182477452
1.922828

Variable Coeff Std Error T-Stat Signif

$* * * * * * * * * * * * * * * * * * * * * * * * * * * * * * * * * * * * * * * * * * * * * * * * * * * * * * * * * * * * * * * * * * * * * * * * * * * * * * * * * *)$

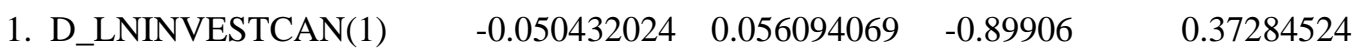

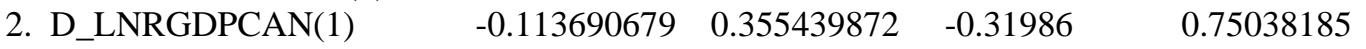

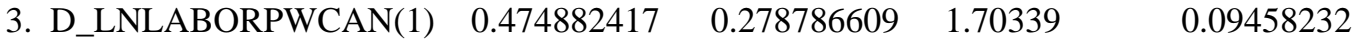

$\begin{array}{lllll}\text { 4. Constant } & -0.356102726 & 0.133132170 & -2.67481 & 0.01002393\end{array}$

$\begin{array}{lllll}\text { 5. EC1 }\{1\} & 0.007145502 & 0.002527700 & 2.82688 & 0.00669809\end{array}$

Dependent Variable LNLABORPWCAN

Annual Data From 1952:01 To 2007:01

Usable Observations $56 \quad$ Degrees of Freedom 51

Mean of Dependent Variable $\quad 0.0141519609$

Std Error of Dependent Variable $\quad 0.0214415084$

Standard Error of Estimate $\quad 0.0193221122$

Sum of Squared Residuals $\quad 0.0190405449$

Durbin-Watson Statistic $\quad 2.047327$

$\begin{array}{lllll}\text { Variable } & \text { Coeff } & \text { Std Error } & \text { T-Stat } & \text { Signif } \\ * * * * * * * * * * * * * * * * * * * * * * * * * * * * * * * * * * * * * * * * * * * * * * * * * * * * * * * * * * * * * * * * * * * * * * * * * * * * * * * * * & \\ \text { 1. D_LNINVESTCAN(1) } & -0.075487647 & 0.057299658 & -1.31742 & 0.19358640 \\ \text { 2. D_LNRGDPCAN(1) } & -0.530893538 & 0.363079081 & -1.46220 & 0.14982214 \\ \text { 3. D_LNLABORPWCAN(1) } & 0.894677336 & 0.284778365 & 3.14166 & 0.00279609 \\ \text { 4. Constant } & -0.201572052 & 0.135993482 & -1.48222 & 0.14443526 \\ \text { 5. EC1 }\{1\} & 0.004110351 & 0.002582026 & 1.59191 & 0.11758511\end{array}$

compute sigma $=\%$ sigma

errors(model=ectmodel,steps $=24$ )

Decomposition of Variance for Series LNINVESTCAN

$\begin{array}{rcccc}\text { Step } & \text { Std Error } & \text { LNINVESTCAN } & \text { LNRGDPCAN } & \text { LNLABORPWCAN } \\ 1 & 0.07795112 & 100.000 & 0.000 & 0.000 \\ 2 & 0.10335218 & 94.712 & 1.457 & 3.831 \\ 3 & 0.12093780 & 88.034 & 3.486 & 8.480 \\ 4 & 0.13544228 & 82.209 & 5.476 & 12.316 \\ 5 & 0.14819123 & 77.308 & 7.352 & 15.340 \\ 6 & 0.15975684 & 73.063 & 9.145 & 17.792 \\ 7 & 0.17046257 & 69.271 & 10.880 & 19.848 \\ 8 & 0.18051818 & 65.810 & 12.572 & 21.618 \\ 9 & 0.19006919 & 62.609 & 14.222 & 23.170 \\ 10 & 0.19922031 & 59.625 & 15.830 & 24.545 \\ 11 & 0.20804862 & 56.831 & 17.394 & 25.775 \\ 12 & 0.21661179 & 54.209 & 18.912 & 26.880 \\ 13 & 0.22495345 & 51.744 & 20.379 & 27.876 \\ 14 & 0.23310688 & 49.426 & 21.796 & 28.778 \\ 15 & 0.24109767 & 47.246 & 23.159 & 29.595 \\ 16 & 0.24894557 & 45.195 & 24.468 & 30.337 \\ 17 & 0.25666591 & 43.265 & 25.724 & 31.011 \\ 18 & 0.26427063 & 41.449 & 26.926 & 31.625 \\ 19 & 0.27176909 & 39.741 & 28.075 & 32.184\end{array}$




$\begin{array}{lllll}20 & 0.27916862 & 38.134 & 29.172 & 32.694 \\ 21 & 0.28647503 & 36.622 & 30.219 & 33.160 \\ 22 & 0.29369296 & 35.198 & 31.217 & 33.585 \\ 23 & 0.30082611 & 33.858 & 32.168 & 33.974 \\ 24 & 0.30787751 & 32.595 & 33.074 & 34.331\end{array}$

Decomposition of Variance for Series LNRGDPCAN

$\begin{array}{rcccc}\text { Step } & \text { Std Error } & \text { LNINVESTCAN } & \text { LNRGDPCAN } & \text { LNLABORPWCAN } \\ 1 & 0.01805139 & 57.996 & 42.004 & 0.000 \\ 2 & 0.02811546 & 38.790 & 58.708 & 2.502 \\ 3 & 0.03685831 & 26.201 & 68.603 & 5.196 \\ 4 & 0.04499285 & 18.401 & 74.491 & 7.108 \\ 5 & 0.05280082 & 13.464 & 78.156 & 8.381 \\ 6 & 0.06044024 & 10.279 & 80.485 & 9.236 \\ 7 & 0.06800082 & 8.231 & 81.945 & 9.824 \\ 8 & 0.07552964 & 6.954 & 82.812 & 10.235 \\ 9 & 0.08304830 & 6.211 & 83.265 & 10.524 \\ 10 & 0.09056381 & 5.844 & 83.428 & 10.727 \\ 11 & 0.09807518 & 5.743 & 83.388 & 10.869 \\ 12 & 0.10557726 & 5.827 & 83.206 & 10.966 \\ 13 & 0.11306293 & 6.041 & 82.928 & 11.031 \\ 14 & 0.12052438 & 6.344 & 82.586 & 11.071 \\ 15 & 0.12795384 & 6.705 & 82.202 & 11.092 \\ 16 & 0.13534396 & 7.104 & 81.795 & 11.101 \\ 17 & 0.14268803 & 7.524 & 81.376 & 11.099 \\ 18 & 0.14998006 & 7.955 & 80.955 & 11.091 \\ 19 & 0.15721483 & 8.387 & 80.536 & 11.077 \\ 20 & 0.16438783 & 8.816 & 80.125 & 11.059 \\ 21 & 0.17149523 & 9.237 & 79.725 & 11.038 \\ 22 & 0.17853385 & 9.647 & 79.338 & 11.015 \\ 23 & 0.18550110 & 10.045 & 78.964 & 10.992 \\ 24 & 0.19239489 & 10.428 & 78.604 & 10.967\end{array}$

Decomposition of Variance for Series LNLABORPWCAN

$\begin{array}{rccrc}\text { Step } & \text { Std Error } & \text { LNINVESTCAN } & \text { LNRGDPCAN } & \text { LNLABORPWCAN } \\ 1 & 0.01843935 & 46.645 & 39.868 & 13.487 \\ 2 & 0.02986993 & 26.318 & 47.999 & 25.683 \\ 3 & 0.03944010 & 17.526 & 50.712 & 31.762 \\ 4 & 0.04753334 & 13.233 & 51.874 & 34.892 \\ 5 & 0.05455736 & 10.811 & 52.515 & 36.674 \\ 6 & 0.06081727 & 9.278 & 52.933 & 37.789 \\ 7 & 0.06651328 & 8.222 & 53.237 & 38.542 \\ 8 & 0.07177572 & 7.447 & 53.473 & 39.080 \\ 9 & 0.07669243 & 6.852 & 53.665 & 39.483 \\ 10 & 0.08132518 & 6.379 & 53.827 & 39.794 \\ 11 & 0.08571927 & 5.992 & 53.967 & 40.041 \\ 12 & 0.08990901 & 5.669 & 54.090 & 40.241 \\ 13 & 0.09392113 & 5.394 & 54.200 & 40.406 \\ 14 & 0.09777694 & 5.157 & 54.298 & 40.544 \\ 15 & 0.10149374 & 4.950 & 54.388 & 40.661 \\ 16 & 0.10508582 & 4.767 & 54.471 & 40.761 \\ 17 & 0.10856516 & 4.605 & 54.547 & 40.848 \\ 18 & 0.11194186 & 4.459 & 54.618 & 40.923 \\ 19 & 0.11522463 & 4.327 & 54.684 & 40.989 \\ 20 & 0.11842094 & 4.208 & 54.745 & 41.047\end{array}$




$\begin{array}{lllll}21 & 0.12153733 & 4.098 & 54.803 & 41.099 \\ 22 & 0.12457954 & 3.998 & 54.857 & 41.145 \\ 23 & 0.12755262 & 3.906 & 54.908 & 41.186 \\ 24 & 0.13046109 & 3.821 & 54.956 & 41.223\end{array}$

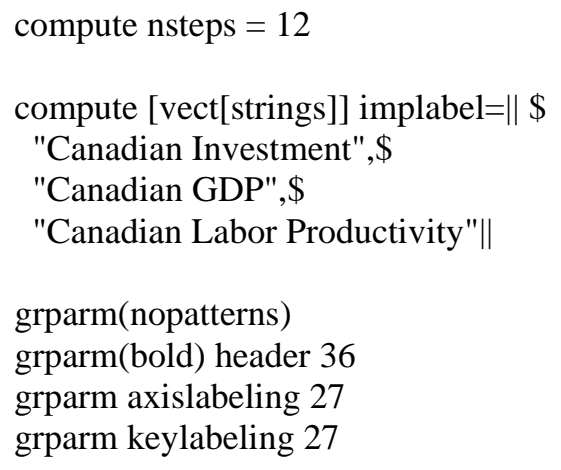




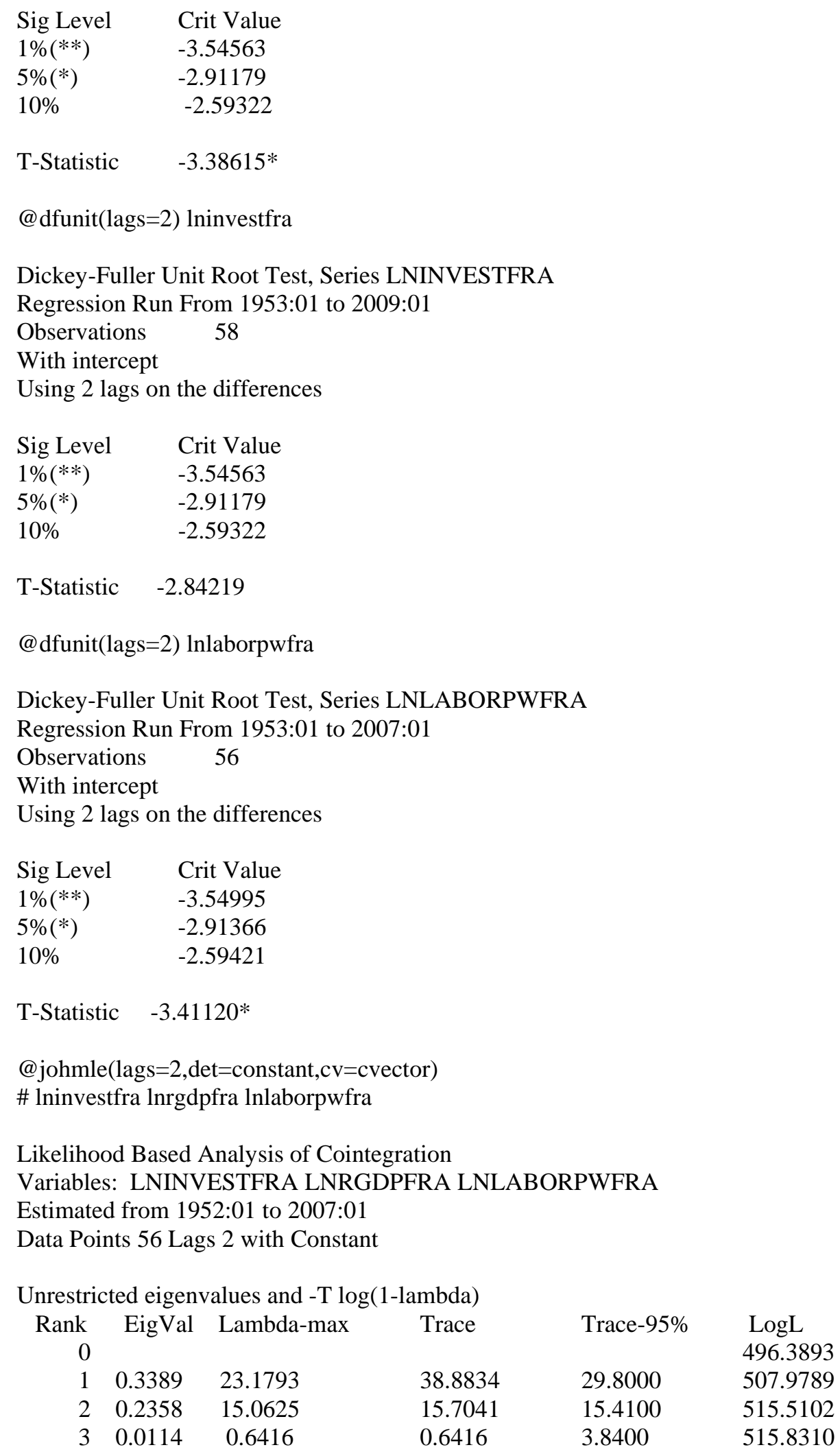

Cointegrating Vector for Largest Eigenvalue LNINVESTFRA LNRGDPFRA LNLABORPWFRA 
equation(coeffs=cvector) ecteq *

\# lninvestfra lnrgdpfra lnlaborpwfra

system(model=ectmodel)

variables lninvestfra lnrgdpfra lnlaborpwfra

lags 1 to 2

det constant

ect ecteq

end(system)

estimate

VAR/System - Estimation by Cointegrated Least Squares

Dependent Variable LNINVESTFRA

Annual Data From 1952:01 To 2007:01

Usable Observations $56 \quad$ Degrees of Freedom 51

Mean of Dependent Variable $\quad 0.0380314919$

Std Error of Dependent Variable $\quad 0.0649053986$

Standard Error of Estimate $\quad 0.0637975882$

Sum of Squared Residuals $\quad 0.2075767451$

Durbin-Watson Statistic $\quad 2.106372$

$\begin{array}{lllll}\text { Variable } & \text { Coeff } & \text { Std Error } & \text { T-Stat } & \text { Signif } \\ \text { ********************************************************************************** } & \\ \text { 1. D_LNINVESTFRA(1) } & 0.256010529 & 0.193960852 & 1.31991 & 0.19275973 \\ \text { 2. D_LNRGDPFRA(1) } & -3.254142536 & 2.427969036 & -1.34027 & 0.18609702 \\ \text { 3. D_LNLABORPWFRA(1) } & 2.813643090 & 2.693656669 & 1.04454 & 0.30115962 \\ \text { 4. Constant } & 0.002764858 & 0.068269655 & 0.04050 & 0.96785338 \\ \text { 5. EC1 }\{1\} & -0.006652164 & 0.008525311 & -0.78028 & 0.43883032\end{array}$

Dependent Variable LNRGDPFRA

Annual Data From 1952:01 To 2007:01

Usable Observations $56 \quad$ Degrees of Freedom 51

Mean of Dependent Variable $\quad 0.0330289620$

Std Error of Dependent Variable $\quad 0.0200021742$

Standard Error of Estimate $\quad 0.0160638039$

Sum of Squared Residuals $\quad 0.0131603356$

Durbin-Watson Statistic $\quad 1.988188$

\begin{tabular}{|c|c|c|c|c|}
\hline Variable & Coeff & Std Error & T-Stat & Signif \\
\hline 1. D LNINVESTFRA(1) & 0031178605 & 0048838039 & 063841 & 052606455 \\
\hline 2. D_LNRGDPFRA(1) & -0.151803812 & 0.611346285 & -0.24831 & 0.80489119 \\
\hline 3. D_LNLABORPWFRA(1) & 0.324379417 & 0.678244646 & 0.47826 & 0.63450678 \\
\hline 4. Constant & -0.015725219 & 0.017189840 & -0.91480 & 0.36460312 \\
\hline 5. EC1 $\{1\}$ & -0.005011266 & 0.002146616 & -2.33450 & 0.02354538 \\
\hline
\end{tabular}

Dependent Variable LNLABORPWFRA

Annual Data From 1952:01 To 2007:01

Usable Observations $56 \quad$ Degrees of Freedom 51

Mean of Dependent Variable $\quad 0.0267315364$

Std Error of Dependent Variable $\quad 0.0195452394$

Standard Error of Estimate $\quad 0.0142884534$ 
Sum of Squared Residuals

Durbin-Watson Statistic
0.0104121549

2.031722

$\begin{array}{lllll}\text { Variable } & \text { Coeff } & \text { Std Error } & \text { T-Stat } & \text { Signif } \\ * * * * * * * * * * * * * * * * * * * * * * * * * * * * * * * * * * * * * * * * * * * * * * * * * * * * * * * * * * * * * * * * * * * * * * * * * * * * * * * * & \\ \text { 1. D_LNINVESTFRA(1) } & 0.039355353 & 0.043440523 & 0.90596 & 0.36921772 \\ \text { 2. D_LNRGDPFRA(1) } & -0.500298431 & 0.543781095 & -0.92004 & 0.36188513 \\ \text { 3. D_LNLABORPWFRA(1) } & 0.603370532 & 0.603285937 & 1.00014 & 0.32196450 \\ \text { 4. Constant } & -0.026952670 & 0.015290042 & -1.76276 & 0.08393251 \\ \text { 5. EC1 }\{1\} & -0.006006485 & 0.001909375 & -3.14579 & 0.00276335\end{array}$

compute sigma $=\%$ sigma

errors(model=ectmodel,steps=24)

Decomposition of Variance for Series LNINVESTFRA

$\begin{array}{rcccc}\text { Step } & \text { Std Error } & \text { LNINVESTFRA } & \text { LNRGDPFRA } & \text { LNLABORPWFRA } \\ 1 & 0.06088290 & 100.000 & 0.000 & 0.000 \\ 2 & 0.09211354 & 98.479 & 0.826 & 0.695 \\ 3 & 0.11585175 & 96.445 & 2.152 & 1.403 \\ 4 & 0.13586177 & 94.706 & 3.402 & 1.892 \\ 5 & 0.15375819 & 93.374 & 4.426 & 2.200 \\ 6 & 0.17030999 & 92.358 & 5.250 & 2.392 \\ 7 & 0.18591777 & 91.558 & 5.928 & 2.514 \\ 8 & 0.20081165 & 90.904 & 6.505 & 2.591 \\ 9 & 0.21513747 & 90.350 & 7.010 & 2.639 \\ 10 & 0.22899539 & 89.868 & 7.464 & 2.669 \\ 11 & 0.24245817 & 89.438 & 7.877 & 2.685 \\ 12 & 0.25558074 & 89.050 & 8.258 & 2.692 \\ 13 & 0.26840559 & 88.694 & 8.614 & 2.692 \\ 14 & 0.28096633 & 88.366 & 8.947 & 2.687 \\ 15 & 0.29328991 & 88.060 & 9.261 & 2.679 \\ 16 & 0.30539828 & 87.774 & 9.559 & 2.667 \\ 17 & 0.31730953 & 87.504 & 9.842 & 2.654 \\ 18 & 0.32903872 & 87.249 & 10.111 & 2.640 \\ 19 & 0.34059855 & 87.007 & 10.368 & 2.624 \\ 20 & 0.35199979 & 86.777 & 10.615 & 2.608 \\ 21 & 0.36325169 & 86.558 & 10.851 & 2.591 \\ 22 & 0.37436223 & 86.349 & 11.077 & 2.574 \\ 23 & 0.38533835 & 86.149 & 11.294 & 2.557 \\ 24 & 0.39618615 & 85.957 & 11.503 & 2.540\end{array}$

Decomposition of Variance for Series LNRGDPFRA

$\begin{array}{rcccc}\text { Step } & \text { Std Error } & \text { LNINVESTFRA } & \text { LNRGDPFRA } & \text { LNLABORPWFRA } \\ 1 & 0.01532991 & 61.069 & 38.931 & 0.000 \\ 2 & 0.02474663 & 67.364 & 32.540 & 0.095 \\ 3 & 0.03208830 & 72.019 & 27.749 & 0.232 \\ 4 & 0.03828435 & 75.735 & 23.917 & 0.347 \\ 5 & 0.04385043 & 78.828 & 20.742 & 0.430 \\ 6 & 0.04905507 & 81.460 & 18.054 & 0.486 \\ 7 & 0.05404016 & 83.720 & 15.756 & 0.524 \\ 8 & 0.05888501 & 85.669 & 13.782 & 0.549 \\ 9 & 0.06363721 & 87.349 & 12.085 & 0.566 \\ 10 & 0.06832707 & 88.794 & 10.629 & 0.578 \\ 11 & 0.07297471 & 90.033 & 9.382 & 0.584 \\ 12 & 0.07759375 & 91.092 & 8.320 & 0.588\end{array}$




$\begin{array}{lllll}13 & 0.08219342 & 91.993 & 7.417 & 0.590 \\ 14 & 0.08677988 & 92.756 & 6.655 & 0.590 \\ 15 & 0.09135716 & 93.398 & 6.014 & 0.588 \\ 16 & 0.09592770 & 93.935 & 5.480 & 0.585 \\ 17 & 0.10049284 & 94.380 & 5.038 & 0.582 \\ 18 & 0.10505311 & 94.745 & 4.676 & 0.578 \\ 19 & 0.10960845 & 95.042 & 4.384 & 0.574 \\ 20 & 0.11415839 & 95.278 & 4.152 & 0.569 \\ 21 & 0.11870218 & 95.463 & 3.972 & 0.565 \\ 22 & 0.12323888 & 95.603 & 3.837 & 0.560 \\ 23 & 0.12776742 & 95.704 & 3.741 & 0.555 \\ 24 & 0.13228666 & 95.771 & 3.679 & 0.550\end{array}$

Decomposition of Variance for Series LNLABORPWFRA

Step Std Error LNINVESTFRA LNRGDPFRA LNLABORPWFRA

$\begin{array}{lllll}1 & 0.01363566 & 58.883 & 36.911 & 4.206\end{array}$

$\begin{array}{lllll}2 & 0.02175781 & 66.418 & 28.003 & 5.579\end{array}$

$\begin{array}{lllll}3 & 0.02811586 & 71.480 & 21.883 & 6.637\end{array}$

$\begin{array}{lllll}4 & 0.03359088 & 75.300 & 17.412 & 7.289\end{array}$

$\begin{array}{lllll}5 & 0.03863499 & 78.361 & 14.024 & 7.614\end{array}$

$\begin{array}{lllll}6 & 0.04346992 & 80.873 & 11.407 & 7.720\end{array}$

$\begin{array}{lllll}7 & 0.04820575 & 82.941 & 9.370 & 7.689\end{array}$

$\begin{array}{lllll}8 & 0.05289959 & 84.635 & 7.791 & 7.574\end{array}$

$\begin{array}{lllll}9 & 0.05758265 & 86.011 & 6.580 & 7.409\end{array}$

$\begin{array}{lllll}10 & 0.06227255 & 87.116 & 5.667 & 7.217\end{array}$

$\begin{array}{lllll}11 & 0.06697921 & 87.992 & 4.998 & 7.010\end{array}$

$\begin{array}{lllll}12 & 0.07170781 & 88.675 & 4.527 & 6.797\end{array}$

$\begin{array}{lllll}13 & 0.07646052 & 89.197 & 4.218 & 6.585\end{array}$

$\begin{array}{lllll}14 & 0.08123750 & 89.585 & 4.038 & 6.377\end{array}$

$\begin{array}{lllll}15 & 0.08603765 & 89.860 & 3.965 & 6.175\end{array}$

$\begin{array}{lllll}16 & 0.09085902 & 90.044 & 3.976 & 5.981\end{array}$

$\begin{array}{lllll}17 & 0.09569914 & 90.151 & 4.054 & 5.795\end{array}$

$\begin{array}{lllll}18 & 0.10055525 & 90.195 & 4.187 & 5.618\end{array}$

$\begin{array}{lllll}19 & 0.10542444 & 90.188 & 4.362 & 5.450\end{array}$

$\begin{array}{lllll}20 & 0.11030377 & 90.139 & 4.570 & 5.291\end{array}$

$\begin{array}{lllll}21 & 0.11519030 & 90.056 & 4.803 & 5.140\end{array}$

$\begin{array}{lllll}22 & 0.12008120 & 89.946 & 5.055 & 4.998\end{array}$

$\begin{array}{lllll}23 & 0.12497373 & 89.815 & 5.322 & 4.864\end{array}$

$\begin{array}{lllll}24 & 0.12986530 & 89.666 & 5.598 & 4.737\end{array}$

compute nsteps $=12$

compute [vect[strings]] implabel=\| \$

"French Investment",\$

"French GDP",\$

"French Labor Productivity"||

grparm(nopatterns)

grparm(bold) header 36

grparm axislabeling 27

grparm keylabeling 27

@VARIRF(model=ectmodel,steps=nsteps,vlables=implabel,byshocks,byvariables) 


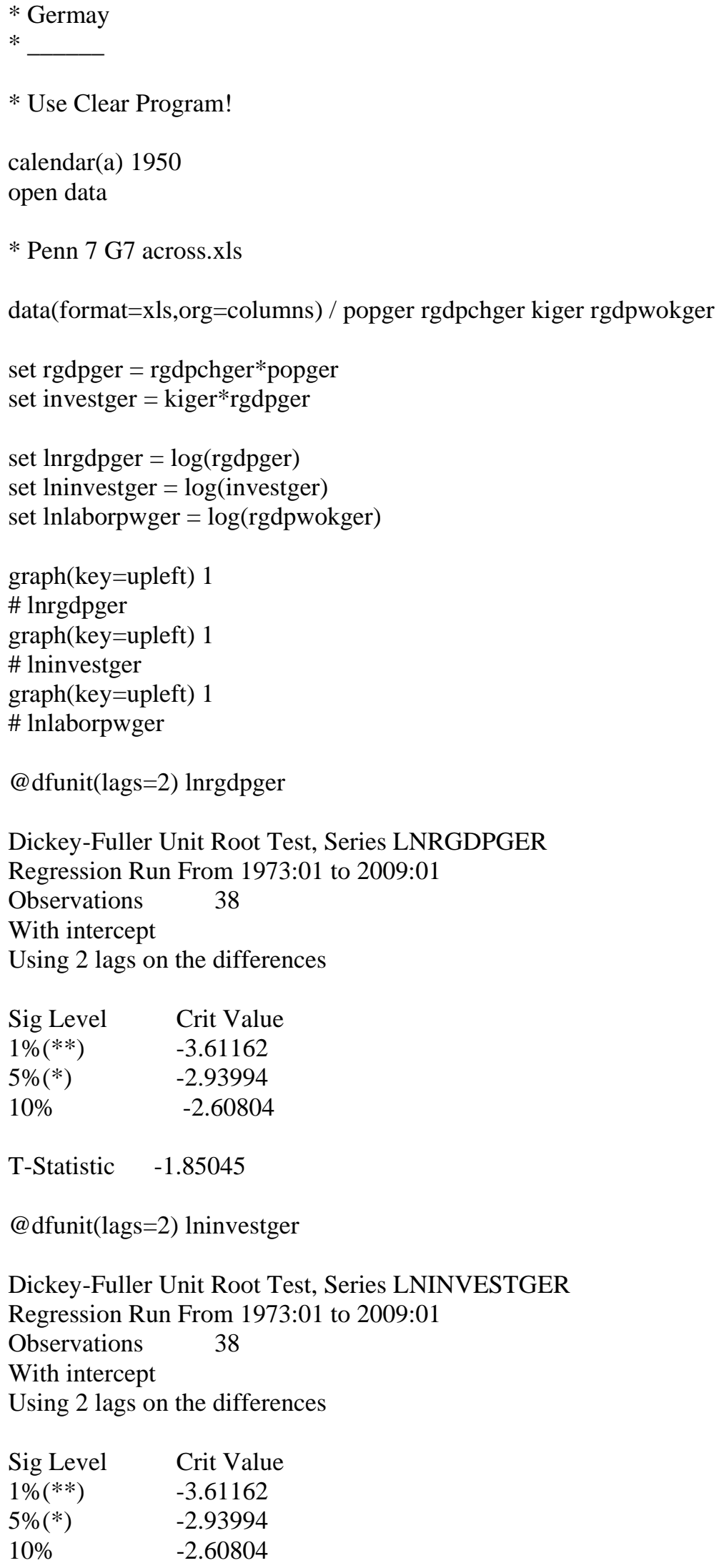




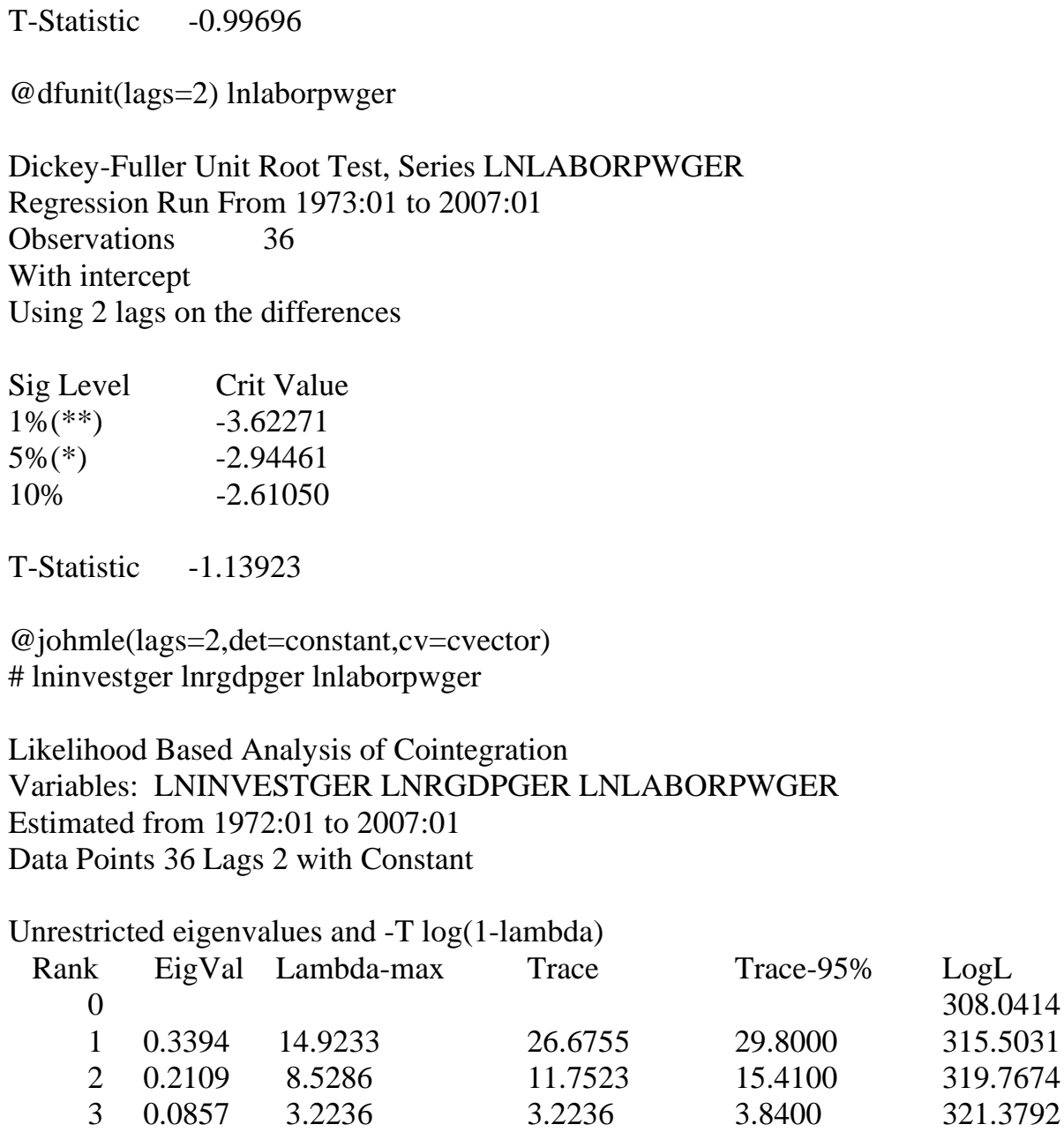

Cointegrating Vector for Largest Eigenvalue LNINVESTGER LNRGDPGER LNLABORPWGER $17.878756-67.052692 \quad 70.850850$

equation(coeffs=cvector) ecteq *

\# lninvestger lnrgdpger lnlaborpwger

system(model=ectmodel)

variables lninvestger lnrgdpger lnlaborpwger

lags 1 to 2

det constant

ect ecteq

end(system)

estimate

VAR/System - Estimation by Cointegrated Least Squares Dependent Variable LNINVESTGER

Annual Data From 1972:01 To 2007:01

Usable Observations $36 \quad$ Degrees of Freedom 31

Mean of Dependent Variable 0.0127517336

Std Error of Dependent Variable 0.0589040019

Standard Error of Estimate $\quad 0.0488489775$ 
Sum of Squared Residuals $\quad 0.0739729007$

Durbin-Watson Statistic $\quad 1.853205$

$\begin{array}{lllll}\text { Variable } & \text { Coeff } & \text { Std Error } & \text { T-Stat } & \text { Signif } \\ * * * * * * * * * * * * * * * * * * * * * * * * * * * * * * * * * * * * * * * * * * * * * * * * * * * * * * * * * * * * * * * * * * * * * * * * * * * * * * * * \\ \text { 1. D_LNINVESTGER(1) } & 0.301579199 & 0.264114392 & 1.14185 & 0.26225959 \\ \text { 2. D_LNRGDPGER(1) } & 0.302969717 & 1.197523248 & 0.25300 & 0.80194079 \\ \text { 3. D_LNLABORPWGER(1) } & 0.034288220 & 1.280029644 & 0.02679 & 0.97880128 \\ \text { 4. Constant } & -5.445992445 & 1.864490684 & -2.92090 & 0.00645583 \\ \text { 5. EC1 }\{1\} & -0.023921905 & 0.008141496 & -2.93827 & 0.00618043\end{array}$

Dependent Variable LNRGDPGER

Annual Data From 1972:01 To 2007:01

Usable Observations $36 \quad$ Degrees of Freedom 31

Mean of Dependent Variable $\quad 0.0208046409$

Std Error of Dependent Variable $\quad 0.0166614783$

Standard Error of Estimate $\quad 0.0148861276$

Sum of Squared Residuals $\quad 0.0068695006$

Durbin-Watson Statistic $\quad 1.889596$

\begin{tabular}{|c|c|c|c|c|}
\hline Variable & Coeff & Std Error & T-Stat & Signif \\
\hline & & & & \\
\hline 1. D_LNINVESTGER(1) & 0.066256132 & 0.080485626 & 0.82320 & 0.41667976 \\
\hline 2. D_LNRGDPGER(1) & 0.498766138 & 0.364930543 & 1.36674 & 0.18153717 \\
\hline 3. D_LNLABORPWGER(1) & -0.172090639 & 0.390073356 & -0.44118 & 0.66214805 \\
\hline 4. Constant & -0.689896998 & 0.568180700 & -1.21422 & 0.23383247 \\
\hline 5. EC1 11$\}$ & -0.003082361 & 0.002481021 & -1.24238 & 0.22341442 \\
\hline
\end{tabular}

Dependent Variable LNLABORPWGER

Annual Data From 1972:01 To 2007:01

Usable Observations $36 \quad$ Degrees of Freedom 31

Mean of Dependent Variable $\quad 0.0149141745$

Std Error of Dependent Variable $\quad 0.0168595302$

Standard Error of Estimate $\quad 0.0161669156$

Sum of Squared Residuals $\quad 0.0081024440$

Durbin-Watson Statistic $\quad 1.920429$

\begin{tabular}{lllll} 
Variable & Coeff & Std Error & \multicolumn{1}{c}{ T-Stat } & Signif \\
$* * * * * * * * * * * * * * * * * * * * * * * * * * * * * * * * * * * * * * * * * * * * * * * * * * * * * * * * * * * * * * * * * * * * * * * * * * * * * * *$ \\
1. D_LNINVESTGER(1) & 0.019773441 & 0.087410532 & 0.22621 & 0.82252033 \\
2. D_LNRGDPGER(1) & 0.221882748 & 0.396328814 & 0.55985 & 0.57960836 \\
3. D_LNLABORPWGER(1) & 0.240516378 & 0.423634891 & 0.56774 & 0.57429775 \\
4. Constant & -0.839661219 & 0.617066419 & -1.36073 & 0.18341047 \\
5. EC1\{1\} & -0.003714935 & 0.002694486 & -1.37872 & 0.17785025
\end{tabular}

compute sigma $=\%$ sigma

errors(model=ectmodel,steps $=24$ )

Decomposition of Variance for Series LNINVESTGER

$\begin{array}{rrrrr}\text { Step } & \text { Std Error } & \text { LNINVESTGER } & \text { LNRGDPGER } & \text { LNLABORPWGER } \\ 1 & 0.04532993 & 100.000 & 0.000 & 0.000 \\ 2 & 0.06365254 & 96.166 & 0.287 & 3.547 \\ 3 & 0.07425999 & 82.875 & 0.488 & 16.637 \\ 4 & 0.08488249 & 64.984 & 0.431 & 34.585 \\ 5 & 0.09639719 & 50.406 & 0.336 & 49.258\end{array}$




$\begin{array}{ccccc}6 & 0.10740765 & 40.654 & 0.308 & 59.038 \\ 7 & 0.11717167 & 34.226 & 0.324 & 65.450 \\ 8 & 0.12571872 & 29.759 & 0.349 & 69.891 \\ 9 & 0.13335441 & 26.456 & 0.369 & 73.175 \\ 10 & 0.14037372 & 23.877 & 0.381 & 75.743 \\ 11 & 0.14697975 & 21.779 & 0.387 & 77.835 \\ 12 & 0.15329043 & 20.023 & 0.390 & 79.588 \\ 13 & 0.15936685 & 18.525 & 0.391 & 81.084 \\ 14 & 0.16523908 & 17.232 & 0.392 & 82.376 \\ 15 & 0.17092347 & 16.105 & 0.393 & 83.502 \\ 16 & 0.17643199 & 15.115 & 0.394 & 84.491 \\ 17 & 0.18177606 & 14.239 & 0.396 & 85.365 \\ 18 & 0.18696737 & 13.460 & 0.397 & 86.144 \\ 19 & 0.19201746 & 12.761 & 0.397 & 86.842 \\ 20 & 0.19693719 & 12.131 & 0.398 & 87.470 \\ 21 & 0.20173640 & 11.561 & 0.399 & 88.040 \\ 22 & 0.20642379 & 11.042 & 0.400 & 88.558 \\ 23 & 0.21100701 & 10.567 & 0.400 & 89.032 \\ 24 & 0.21549280 & 10.132 & 0.401 & 89.467\end{array}$

Decomposition of Variance for Series LNRGDPGER

$\begin{array}{rcccc}\text { Step } & \text { Std Error } & \text { LNINVESTGER } & \text { LNRGDPGER } & \text { LNLABORPWGER } \\ 1 & 0.01381374 & 76.017 & 23.983 & 0.000 \\ 2 & 0.02355831 & 74.720 & 23.848 & 1.432 \\ 3 & 0.03069038 & 69.869 & 24.865 & 5.266 \\ 4 & 0.03609458 & 63.406 & 25.688 & 10.906 \\ 5 & 0.04053694 & 57.035 & 25.995 & 16.970 \\ 6 & 0.04439575 & 51.704 & 25.961 & 22.336 \\ 7 & 0.04784490 & 47.603 & 25.809 & 26.588 \\ 8 & 0.05099354 & 44.553 & 25.658 & 29.789 \\ 9 & 0.05392306 & 42.283 & 25.544 & 32.172 \\ 10 & 0.05668963 & 40.554 & 25.466 & 33.980 \\ 11 & 0.05932786 & 39.188 & 25.414 & 35.399 \\ 12 & 0.06185806 & 38.066 & 25.377 & 36.557 \\ 13 & 0.06429284 & 37.117 & 25.348 & 37.535 \\ 14 & 0.06664126 & 36.297 & 25.324 & 38.379 \\ 15 & 0.06891083 & 35.578 & 25.303 & 39.119 \\ 16 & 0.07110825 & 34.943 & 25.284 & 39.773 \\ 17 & 0.07323964 & 34.379 & 25.267 & 40.354 \\ 18 & 0.07531051 & 33.875 & 25.252 & 40.873 \\ 19 & 0.07732575 & 33.423 & 25.238 & 41.339 \\ 20 & 0.07928970 & 33.016 & 25.225 & 41.759 \\ 21 & 0.08120611 & 32.646 & 25.213 & 42.140 \\ 22 & 0.08307833 & 32.310 & 25.203 & 42.487 \\ 23 & 0.08490929 & 32.003 & 25.193 & 42.804 \\ 24 & 0.08670160 & 31.720 & 25.185 & 43.095\end{array}$

Decomposition of Variance for Series LNLABORPWGER

$\begin{array}{rlccc}\text { Step } & \text { Std Error } & \text { LNINVESTGER } & \text { LNRGDPGER } & \text { LNLABORPWGER } \\ 1 & 0.01500226 & 62.327 & 14.517 & 23.156 \\ 2 & 0.02410011 & 63.582 & 18.875 & 17.544 \\ 3 & 0.02995768 & 63.315 & 23.183 & 13.502 \\ 4 & 0.03376238 & 62.044 & 27.068 & 10.888 \\ 5 & 0.03646576 & 60.357 & 30.309 & 9.334 \\ 6 & 0.03861509 & 58.701 & 32.915 & 8.384\end{array}$




$\begin{array}{rcccc}7 & 0.04049164 & 57.283 & 35.006 & 7.711 \\ 8 & 0.04223771 & 56.144 & 36.700 & 7.156 \\ 9 & 0.04392021 & 55.243 & 38.091 & 6.666 \\ 10 & 0.04556317 & 54.522 & 39.253 & 6.226 \\ 11 & 0.04716963 & 53.926 & 40.242 & 5.833 \\ 12 & 0.04873595 & 53.417 & 41.099 & 5.484 \\ 13 & 0.05025903 & 52.971 & 41.854 & 5.175 \\ 14 & 0.05173842 & 52.573 & 42.526 & 4.901 \\ 15 & 0.05317585 & 52.214 & 43.129 & 4.657 \\ 16 & 0.05457423 & 51.890 & 43.672 & 4.438 \\ 17 & 0.05593672 & 51.596 & 44.163 & 4.241 \\ 18 & 0.05726630 & 51.329 & 44.609 & 4.062 \\ 19 & 0.05856551 & 51.085 & 45.017 & 3.898 \\ 20 & 0.05983650 & 50.861 & 45.390 & 3.749 \\ 21 & 0.06108111 & 50.656 & 45.733 & 3.611 \\ 22 & 0.06230092 & 50.467 & 46.049 & 3.484 \\ 23 & 0.06349734 & 50.292 & 46.341 & 3.367 \\ 24 & 0.06467165 & 50.130 & 46.613 & 3.258\end{array}$

compute nsteps $=12$

compute [vect[strings]] implabel=\| \$

"German Investment",\$

"German GDP",\$

"German Labor Productivity"||

grparm(nopatterns)

grparm(bold) header 36

grparm axislabeling 27

grparm keylabeling 27

@VARIRF(model=ectmodel,steps=nsteps,vlables=implabel,byshocks,byvariables)

* Italy

$*$

* Use Clear Program!

calendar(a) 1950

open data

* Penn 7 G7 across.xls

data(format=xls,org=columns) / popita rgdpchita kiita rgdpwokita

set rgdpita = rgdpchita*popita

set investita $=$ kiita ${ }^{*}$ rgdpita

set lnrgdpita $=\log ($ rgdpita $)$

set lninvestita $=\log ($ investita $)$

set lnlaborpwita $=\log ($ rgdpwokita $)$

graph(key=upleft) 1 


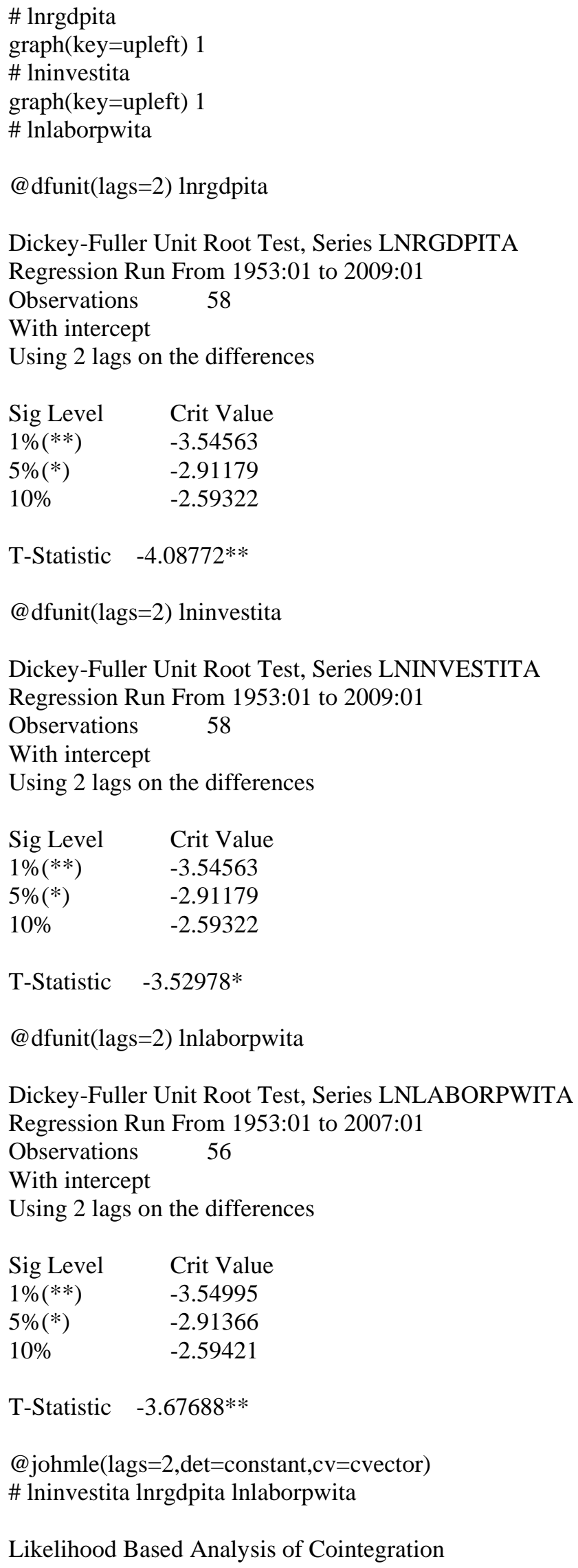


Variables: LNINVESTITA LNRGDPITA LNLABORPWITA

Estimated from 1952:01 to 2007:01

Data Points 56 Lags 2 with Constant

\begin{tabular}{rccccl}
\multicolumn{6}{c}{ Unrestricted eigenvalues and -T log(1-lambda) } \\
Rank & EigVal & Lambda-max & Trace & Trace-95\% & LogL \\
0 & & & & & 437.9269 \\
1 & 0.2793 & 18.3396 & 38.0761 & 29.8000 & 447.0967 \\
2 & 0.1977 & 12.3338 & 19.7365 & 15.4100 & 453.2635 \\
3 & 0.1238 & 7.4027 & 7.4027 & 3.84004 & 56.9649
\end{tabular}

Cointegrating Vector for Largest Eigenvalue LNINVESTITA LNRGDPITA LNLABORPWITA

$$
7.237020-22.444500 \quad 13.677178
$$

equation(coeffs=cvector) ecteq *

\# lninvestita lnrgdpita lnlaborpwita

system(model=ectmodel)

variables lninvestita lnrgdpita Inlaborpwita

lags 1 to 2

det constant

ect ecteq

end(system)

estimate

VAR/System - Estimation by Cointegrated Least Squares

Dependent Variable LNINVESTITA

Annual Data From 1952:01 To 2007:01

Usable Observations $56 \quad$ Degrees of Freedom 51

Mean of Dependent Variable $\quad 0.0372344148$

Std Error of Dependent Variable $\quad 0.0745349742$

Standard Error of Estimate $\quad 0.0755176831$

Sum of Squared Residuals $\quad 0.2908489434$

Durbin-Watson Statistic $\quad 1.991670$

\begin{tabular}{|c|c|c|c|c|}
\hline Variable & Coeff & Std Error & T-Stat & Signif \\
\hline 1. D_LNINVESTITA(1) & -0.356056155 & 0.271443537 & -1.31171 & 0.19549109 \\
\hline 2. D_LNRGDPITA(1) & 1.265867215 & 1.565319083 & 0.80870 & 0.42244663 \\
\hline 3. D_LNLABORPWITA(1) & 0.233665277 & 1.064766381 & 0.21945 & 0.82717382 \\
\hline 4. Constant & -1.009981873 & 1.495459063 & -0.67537 & 0.50249417 \\
\hline 5. $\operatorname{EC} 1\{1\}$ & -0.006913807 & 0.010091475 & -0.68511 & 0.49637446 \\
\hline
\end{tabular}

Dependent Variable LNRGDPITA

Annual Data From 1952:01 To 2007:01

Usable Observations $56 \quad$ Degrees of Freedom 51

Mean of Dependent Variable $\quad 0.0336026785$

Std Error of Dependent Variable $\quad 0.0237686300$

Standard Error of Estimate $\quad 0.0187735816$

Sum of Squared Residuals $\quad 0.0179748156$

Durbin-Watson Statistic $\quad 2.020743$

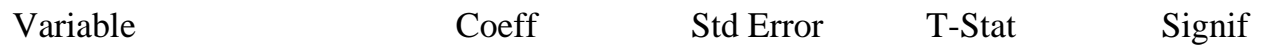




\begin{tabular}{|c|c|c|c|c|}
\hline 1. D_LNINVESTITA(1) & -0.169732796 & 0.067480452 & -2.51529 & 0.01508640 \\
\hline 2. D_LNRGDPITA(1) & 0.624053776 & 0.389135952 & 1.60369 & 0.11495804 \\
\hline 3. D_LNLABORPWITA(1) & 0.084786160 & 0.264699309 & 0.32031 & 0.75004112 \\
\hline 4. Constant & 0.460158610 & 0.371768857 & 1.23775 & 0.22147522 \\
\hline 5. EC1 $1\{1\}$ & 0.003045023 & 0.002508725 & 1.21377 & 0.23042557 \\
\hline
\end{tabular}

Dependent Variable LNLABORPWITA

Annual Data From 1952:01 To 2007:01

Usable Observations $56 \quad$ Degrees of Freedom 51

Mean of Dependent Variable $\quad 0.0323209679$

Std Error of Dependent Variable $\quad 0.0268766344$

Standard Error of Estimate $\quad 0.0195529250$

Sum of Squared Residuals $\quad 0.0194981607$

Durbin-Watson Statistic $\quad 2.058115$

$\begin{array}{llccc}\text { Variable } & \text { Coeff } & \text { Std Error } & \text { T-Stat } & \text { Signif } \\ * * * * * * * * * * * * * * * * * * * * * * * * * * * * * * * * * * * * * * * * * * * * * * * * * * * * * * * * * * * * * * * * * * * * * & \\ \text { 1. D_LNINVESTITA(1) } & -0.182567884 & 0.070281753 & -2.59766 & 0.01223785 \\ \text { 2. D_LNRGDPITA(1) } & 0.293532264 & 0.405290064 & 0.72425 & 0.47222034 \\ \text { 3. D_LNLABORPWITA(1) } & 0.434505956 & 0.275687711 & 1.57608 & 0.12119083 \\ \text { 4. Constant } & 0.627178955 & 0.387202013 & 1.61977 & 0.11144921 \\ \text { 5. EC1 }\{1\} & 0.004196021 & 0.002612870 & 1.60591 & 0.11446960\end{array}$

compute sigma $=\%$ sigma

errors(model=ectmodel,steps $=24$ )

Decomposition of Variance for Series LNINVESTITA

$\begin{array}{rrrcc}\text { Step } & \text { Std Error } & \text { LNINVESTITA } & \text { LNRGDPITA } & \text { LNLABORPWITA } \\ 1 & 0.07206755 & 100.000 & 0.000 & 0.000 \\ 2 & 0.09970857 & 98.574 & 1.411 & 0.016 \\ 3 & 0.12132079 & 97.346 & 2.613 & 0.041 \\ 4 & 0.14014604 & 96.523 & 3.403 & 0.074 \\ 5 & 0.15728560 & 95.978 & 3.905 & 0.117 \\ 6 & 0.17326935 & 95.604 & 4.229 & 0.167 \\ 7 & 0.18840322 & 95.334 & 4.441 & 0.226 \\ 8 & 0.20288397 & 95.129 & 4.580 & 0.291 \\ 9 & 0.21684716 & 94.967 & 4.671 & 0.362 \\ 10 & 0.23039069 & 94.832 & 4.728 & 0.440 \\ 11 & 0.24358780 & 94.717 & 4.761 & 0.522 \\ 12 & 0.25649474 & 94.616 & 4.775 & 0.609 \\ 13 & 0.26915566 & 94.523 & 4.777 & 0.700 \\ 14 & 0.28160581 & 94.437 & 4.768 & 0.794 \\ 15 & 0.29387377 & 94.356 & 4.752 & 0.892 \\ 16 & 0.30598296 & 94.278 & 4.730 & 0.992 \\ 17 & 0.31795282 & 94.202 & 4.703 & 1.095 \\ 18 & 0.32979959 & 94.127 & 4.673 & 1.199 \\ 19 & 0.34153696 & 94.054 & 4.640 & 1.306 \\ 20 & 0.35317658 & 93.981 & 4.605 & 1.414 \\ 21 & 0.36472835 & 93.908 & 4.569 & 1.523 \\ 22 & 0.37620080 & 93.836 & 4.531 & 1.633 \\ 23 & 0.38760126 & 93.763 & 4.493 & 1.744 \\ 24 & 0.39893611 & 93.691 & 4.454 & 1.856\end{array}$

Decomposition of Variance for Series LNRGDPITA 


$\begin{array}{rcccc}\text { Step } & \text { Std Error } & \text { LNINVESTITA } & \text { LNRGDPITA } & \text { LNLABORPWITA } \\ 1 & 0.01791588 & 82.469 & 17.531 & 0.000 \\ 2 & 0.02747505 & 70.859 & 28.972 & 0.169 \\ 3 & 0.03603119 & 66.449 & 32.971 & 0.580 \\ 4 & 0.04417756 & 65.221 & 33.580 & 1.971 \\ 6 & 0.06024290 & 66.007 & 31.154 & 2.840 \\ 7 & 0.06839979 & 66.865 & 29.374 & 3.761 \\ 8 & 0.07671406 & 67.751 & 27.549 & 4.700 \\ 9 & 0.08521047 & 68.592 & 25.775 & 5.633 \\ 10 & 0.09390104 & 69.357 & 24.099 & 6.545 \\ 11 & 0.10279014 & 70.038 & 22.538 & 7.424 \\ 12 & 0.11187741 & 70.635 & 21.100 & 8.266 \\ 13 & 0.12115960 & 71.154 & 19.780 & 9.067 \\ 14 & 0.13063165 & 71.602 & 18.572 & 9.825 \\ 15 & 0.14028744 & 71.988 & 17.469 & 10.543 \\ 16 & 0.15012025 & 72.320 & 16.461 & 11.857 \\ 18 & 0.17028867 & 72.845 & 14.697 & 12.458 \\ 19 & 0.18061006 & 73.051 & 13.926 & 13.023 \\ 20 & 0.19108022 & 73.226 & 13.218 & 13.556 \\ 21 & 0.20169233 & 73.374 & 12.567 & 14.058 \\ 22 & 0.21243980 & 73.499 & 11.968 & 14.532 \\ 23 & 0.22331624 & 73.604 & 11.416 & 14.979 \\ 24 & 0.23431551 & 73.692 & 10.907 & 15.401\end{array}$

Decomposition of Variance for Series LNLABORPWITA

$\begin{array}{rlccc}\text { Step } & \text { Std Error } & \text { LNINVESTITA } & \text { LNRGDPITA } & \text { LNLABORPWITA } \\ 1 & 0.01865962 & 56.316 & 20.759 & 22.925 \\ 2 & 0.03000370 & 41.036 & 30.362 & 28.602 \\ 3 & 0.04043455 & 36.148 & 32.368 & 31.484 \\ 4 & 0.05046394 & 35.125 & 31.640 & 33.235 \\ 5 & 0.06039418 & 35.683 & 29.929 & 34.389 \\ 6 & 0.07039543 & 36.908 & 27.917 & 35.175 \\ 7 & 0.08055988 & 38.395 & 25.886 & 35.719 \\ 8 & 0.09093611 & 39.949 & 23.956 & 36.095 \\ 9 & 0.10154829 & 41.478 & 22.171 & 36.351 \\ 10 & 0.11240660 & 42.936 & 20.545 & 36.519 \\ 11 & 0.12351304 & 44.303 & 19.074 & 36.623 \\ 12 & 0.13486473 & 45.574 & 17.747 & 36.680 \\ 13 & 0.14645590 & 46.749 & 16.551 & 36.700 \\ 14 & 0.15827911 & 47.833 & 15.473 & 36.694 \\ 15 & 0.17032593 & 48.831 & 14.501 & 36.668 \\ 16 & 0.18258750 & 49.751 & 13.622 & 36.627 \\ 17 & 0.19505475 & 50.599 & 12.827 & 36.575 \\ 18 & 0.20771868 & 51.381 & 12.104 & 36.515 \\ 19 & 0.22057040 & 52.104 & 11.447 & 36.449 \\ 20 & 0.23360126 & 52.772 & 10.848 & 36.380 \\ 21 & 0.24680289 & 53.392 & 10.301 & 36.308 \\ 22 & 0.26016718 & 53.967 & 9.799 & 36.234 \\ 23 & 0.27368638 & 54.501 & 9.339 & 36.160 \\ 24 & 0.28735301 & 54.999 & 8.915 & 36.086\end{array}$

compute nsteps $=12$

compute [vect[strings]] implabel=|| \$

"Italian Investment",\$ 


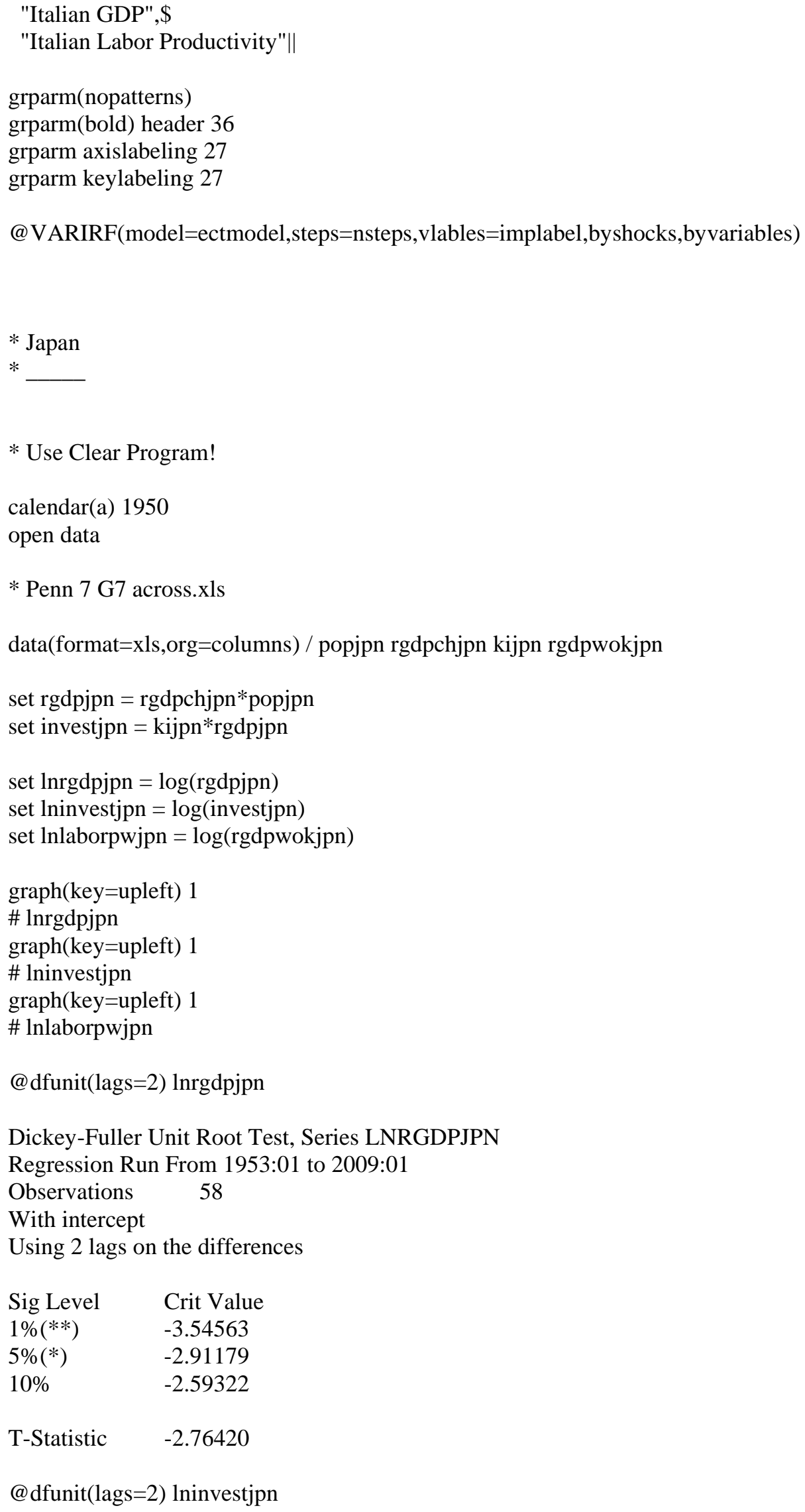


Dickey-Fuller Unit Root Test, Series LNINVESTJPN

Regression Run From 1953:01 to 2009:01

Observations

58

With intercept

Using 2 lags on the differences

$\begin{array}{ll}\text { Sig Level } & \text { Crit Value } \\ 1 \%(* *) & -3.54563 \\ 5 \%(*) & -2.91179 \\ 10 \% & -2.59322\end{array}$

T-Statistic $\quad-4.22033^{* *}$

@dfunit(lags=2) lnlaborpwjpn

Dickey-Fuller Unit Root Test, Series LNLABORPWJPN

Regression Run From 1953:01 to 2007:01

Observations $\quad 56$

With intercept

Using 2 lags on the differences

$\begin{array}{ll}\text { Sig Level } & \text { Crit Value } \\ 1 \%(* *) & -3.54995 \\ 5 \%(*) & -2.91366 \\ 10 \% & -2.59421\end{array}$

T-Statistic $\quad-2.45773$

@johmle(lags=2,det $=$ constant,cv=cvector $)$

\# lninvestjpn lnrgdpjpn lnlaborpwjpn

Likelihood Based Analysis of Cointegration

Variables: LNINVESTJPN LNRGDPJPN LNLABORPWJPN

Estimated from 1952:01 to 2007:01

Data Points 56 Lags 2 with Constant

Unrestricted eigenvalues and - $\mathrm{T} \log (1$-lambda)

$\begin{array}{rcclll}\text { Rank } & \text { EigVal } & \text { Lambda-max } & \text { Trace } & \text { Trace-95\% } & \text { LogL } \\ 0 & & & & & 421.6950 \\ 1 & 0.3166 & 21.3202 & 30.1525 & 29.8000 & 432.3551 \\ 2 & 0.1049 & 6.2055 & 8.8324 & 15.4100 & 435.4578 \\ 3 & 0.0458 & 2.6269 & 2.6269 & 3.8400 & 436.7712\end{array}$

Cointegrating Vector for Largest Eigenvalue LNINVESTJPN LNRGDPJPN LNLABORPWJPN

$$
3.722895-9.596783 \quad 3.941350
$$

equation(coeffs=cvector) ecteq *

\# lninvestjpn lnrgdpjpn lnlaborpwjpn

system(model=ectmodel)

variables lninvestjpn lnrgdpjpn lnlaborpwjpn

lags 1 to 2

det constant 
ect ecteq

end(system)

estimate

VAR/System - Estimation by Cointegrated Least Squares

Dependent Variable LNINVESTJPN

Annual Data From 1952:01 To 2007:01

Usable Observations $56 \quad$ Degrees of Freedom 51

Mean of Dependent Variable $\quad 0.0536977230$

Std Error of Dependent Variable $\quad 0.0878229174$

Standard Error of Estimate $\quad 0.0771549974$

Sum of Squared Residuals $\quad 0.3035975749$

Durbin-Watson Statistic $\quad 1.324520$

Variable Coeff Std Error T-Stat Signif

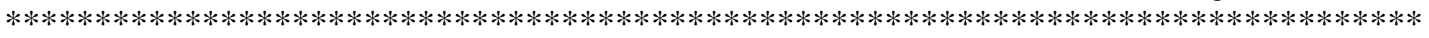

1. D_LNINVESTJPN(1) $\quad-0.138613254 \quad 0.157408875 \quad-0.88059 \quad 0.38266881$

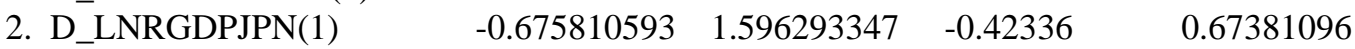

3. D_LNLABORPWJPN(1) $\quad 2.014857096 \quad 1.567418267 \quad 1.28546 \quad 0.20443747$

$\begin{array}{lllll}\text { 4. Constant } & 0.670656986 & 0.759724292 & 0.88276 & 0.38150613\end{array}$

$\begin{array}{lllll}\text { 5. EC1 }\{1\} & 0.009138034 & 0.010310270 & 0.88630 & 0.37961403\end{array}$

Dependent Variable LNRGDPJPN

Annual Data From 1952:01 To 2007:01

Usable Observations $56 \quad$ Degrees of Freedom 51

Mean of Dependent Variable $\quad 0.0482705703$

Std Error of Dependent Variable $\quad 0.0375427836$

Standard Error of Estimate $\quad 0.0223055239$

Sum of Squared Residuals $\quad 0.0253743563$

Durbin-Watson Statistic $\quad 1.787903$

\begin{tabular}{|c|c|c|c|c|}
\hline Variable & Coeff & Std Error & T-Stat & Signif \\
\hline 1. D LNINVESTJPN(1) & 0.032007918 & 0.045506935 & 0.70336 & 0.48502800 \\
\hline 2. D-LNRGDPJPN(1) & -0.632947588 & 0.461488700 & -1.37153 & 0.17621276 \\
\hline 3. D_LNLABORPWJPN(1) & 1.033180454 & 0.453140909 & 2.28004 & 0.02681831 \\
\hline 4. Constant & 0.852347205 & 0.219636432 & 3.88072 & 0.00030012 \\
\hline 5. EC1 11$\}$ & 0.011380114 & 0.002980701 & 3.81793 & 0.00036593 \\
\hline
\end{tabular}

Dependent Variable LNLABORPWJPN

Annual Data From 1952:01 To 2007:01

Usable Observations $56 \quad$ Degrees of Freedom 51

Mean of Dependent Variable $\quad 0.0386879453$

Std Error of Dependent Variable $\quad 0.0335539113$

Standard Error of Estimate $\quad 0.0212642800$

Sum of Squared Residuals $\quad 0.0230606498$

Durbin-Watson Statistic $\quad 1.851307$

\begin{tabular}{|c|c|c|c|c|}
\hline Variable & Coeff & Std Error & T-Stat & Signif \\
\hline 1. D LNINVESTJPN(1) & 0.036280394 & 0.043382626 & 0.83629 & 0.40689394 \\
\hline 2. D_LNRGDPJPN(1) & -0.974647071 & 0.439945951 & -2.21538 & 0.03122543 \\
\hline 3. D_LNLABORPWJPN(1) & 1.328934602 & 0.431987843 & 3.07632 & 0.00336593 \\
\hline 4. Constant & 0.757620337 & 0.209383586 & 3.61834 & 0.00068043 \\
\hline
\end{tabular}


$\begin{array}{lllll}\text { 5. EC1 }\{1\} & 0.010122773 & 0.002841559 & 3.56240 & 0.00080731\end{array}$

compute sigma $=\%$ sigma

errors(model=ectmodel,steps=24)

Decomposition of Variance for Series LNINVESTJPN

$\begin{array}{rlrcc}\text { Step } & \text { Std Error } & \text { LNINVESTJPN } & \text { LNRGDPJPN } & \text { LNLABORPWJPN } \\ 1 & 0.07363006 & 100.000 & 0.000 & 0.000 \\ 2 & 0.11265130 & 96.672 & 2.121 & 3.148 \\ 4 & 0.18055017 & 92.542 & 2.613 & 4.845 \\ 5 & 0.20980773 & 91.389 & 2.496 & 6.115 \\ 6 & 0.23709782 & 90.641 & 2.329 & 7.030 \\ 7 & 0.26300562 & 90.155 & 2.151 & 7.694 \\ 8 & 0.28792101 & 89.832 & 1.978 & 8.190 \\ 9 & 0.31210757 & 89.613 & 1.815 & 8.573 \\ 10 & 0.33574436 & 89.460 & 1.663 & 8.877 \\ 11 & 0.35895662 & 89.351 & 1.524 & 9.125 \\ 12 & 0.38183463 & 89.271 & 1.396 & 9.332 \\ 13 & 0.40444530 & 89.212 & 1.280 & 9.508 \\ 14 & 0.42683936 & 89.167 & 1.173 & 9.660 \\ 15 & 0.44905597 & 89.132 & 1.077 & 9.791 \\ 16 & 0.47112573 & 89.103 & 0.989 & 9.907 \\ 17 & 0.49307279 & 89.080 & 0.910 & 10.010 \\ 18 & 0.51491633 & 89.059 & 0.839 & 10.102 \\ 19 & 0.53667169 & 89.041 & 0.774 & 10.184 \\ 20 & 0.55835111 & 89.025 & 0.716 & 10.259 \\ 21 & 0.57996444 & 89.009 & 0.664 & 10.327 \\ 22 & 0.60151956 & 88.994 & 0.617 & 10.388 \\ 23 & 0.62302276 & 88.980 & 0.576 & 10.445 \\ 24 & 0.64447905 & 88.965 & 0.538 & 10.497\end{array}$

Decomposition of Variance for Series LNRGDPJPN

$\begin{array}{rlccc}\text { Step } & \text { Std Error } & \text { LNINVESTJPN } & \text { LNRGDPJPN } & \text { LNLABORPWJPN } \\ 1 & 0.02128646 & 55.861 & 44.139 & 0.000 \\ 2 & 0.03785036 & 60.605 & 36.440 & 2.955 \\ 3 & 0.05265226 & 62.125 & 31.347 & 6.528 \\ 4 & 0.06605934 & 63.264 & 27.398 & 9.338 \\ 5 & 0.07846491 & 64.426 & 24.256 & 11.317 \\ 6 & 0.09021390 & 65.655 & 21.666 & 12.679 \\ 7 & 0.10155451 & 66.903 & 19.472 & 13.625 \\ 8 & 0.11265275 & 68.127 & 17.577 & 14.297 \\ 9 & 0.12361597 & 69.298 & 15.917 & 14.784 \\ 10 & 0.13451344 & 70.402 & 14.452 & 15.146 \\ 11 & 0.14539042 & 71.432 & 13.150 & 15.418 \\ 12 & 0.15627695 & 72.387 & 11.989 & 15.625 \\ 13 & 0.16719318 & 73.269 & 10.950 & 15.781 \\ 14 & 0.17815264 & 74.083 & 10.018 & 15.899 \\ 15 & 0.18916431 & 74.833 & 9.180 & 15.988 \\ 16 & 0.20023390 & 75.522 & 8.425 & 16.053 \\ 17 & 0.21136476 & 76.158 & 7.744 & 16.099 \\ 18 & 0.22255853 & 76.742 & 7.129 & 16.129 \\ 19 & 0.23381558 & 77.281 & 6.572 & 16.148 \\ 20 & 0.24513532 & 77.777 & 6.067 & 16.156 \\ 21 & 0.25651646 & 78.234 & 5.610 & 16.156 \\ 22 & 0.26795722 & 78.656 & 5.194 & 16.150\end{array}$




$\begin{array}{lllll}23 & 0.27945541 & 79.046 & 4.816 & 16.138 \\ 24 & 0.29100859 & 79.407 & 4.472 & 16.121\end{array}$

Decomposition of Variance for Series LNLABORPWJPN

$\begin{array}{rlccc}\text { Step } & \text { Std Error } & \text { LNINVETJPN } & \text { LNRGDPJPN } & \text { LNLABORPWJPN } \\ 1 & 0.02029279 & 48.428 & 42.726 & 8.845 \\ 2 & 0.03685076 & 49.832 & 32.435 & 17.733 \\ 3 & 0.05167292 & 49.424 & 26.893 & 23.683 \\ 4 & 0.06495655 & 49.490 & 23.128 & 27.382 \\ 5 & 0.07707469 & 50.012 & 20.373 & 29.615 \\ 6 & 0.08839028 & 50.843 & 18.217 & 30.940 \\ 7 & 0.09917247 & 51.847 & 16.446 & 31.707 \\ 8 & 0.10960441 & 52.933 & 14.942 & 32.124 \\ 9 & 0.11980657 & 54.044 & 13.636 & 32.320 \\ 10 & 0.12985843 & 55.146 & 12.485 & 32.369 \\ 11 & 0.13981347 & 56.221 & 11.460 & 32.319 \\ 12 & 0.14970860 & 57.258 & 10.541 & 32.201 \\ 13 & 0.15956987 & 58.251 & 9.714 & 32.035 \\ 14 & 0.16941602 & 59.197 & 8.966 & 31.836 \\ 15 & 0.17926061 & 60.098 & 8.288 & 31.614 \\ 16 & 0.18911351 & 60.952 & 7.672 & 31.376 \\ 17 & 0.19898183 & 61.761 & 7.111 & 31.127 \\ 18 & 0.20887061 & 62.528 & 6.600 & 30.872 \\ 19 & 0.21878332 & 63.253 & 6.133 & 30.614 \\ 20 & 0.22872224 & 63.940 & 5.706 & 30.354 \\ 21 & 0.23868871 & 64.589 & 5.315 & 30.096 \\ 22 & 0.24868333 & 65.203 & 4.957 & 29.840 \\ 23 & 0.25870616 & 65.785 & 4.628 & 29.587 \\ 24 & 0.26875683 & 66.336 & 4.326 & 29.338\end{array}$

compute nsteps $=12$

compute [vect[strings]] implabel=|| \$

"Japanese Investment",\$

"Japanese GDP",\$

"Japanese Labor Productivity"||

grparm(nopatterns)

grparm(bold) header 36

grparm axislabeling 27

grparm keylabeling 27

@VARIRF(model=ectmodel,steps=nsteps,vlables=implabel,byshocks,byvariables)

* United Kingdom

$*$

* Use Clear Program!

calendar(a) 1950

open data

* Penn 7 G7 across.xls 


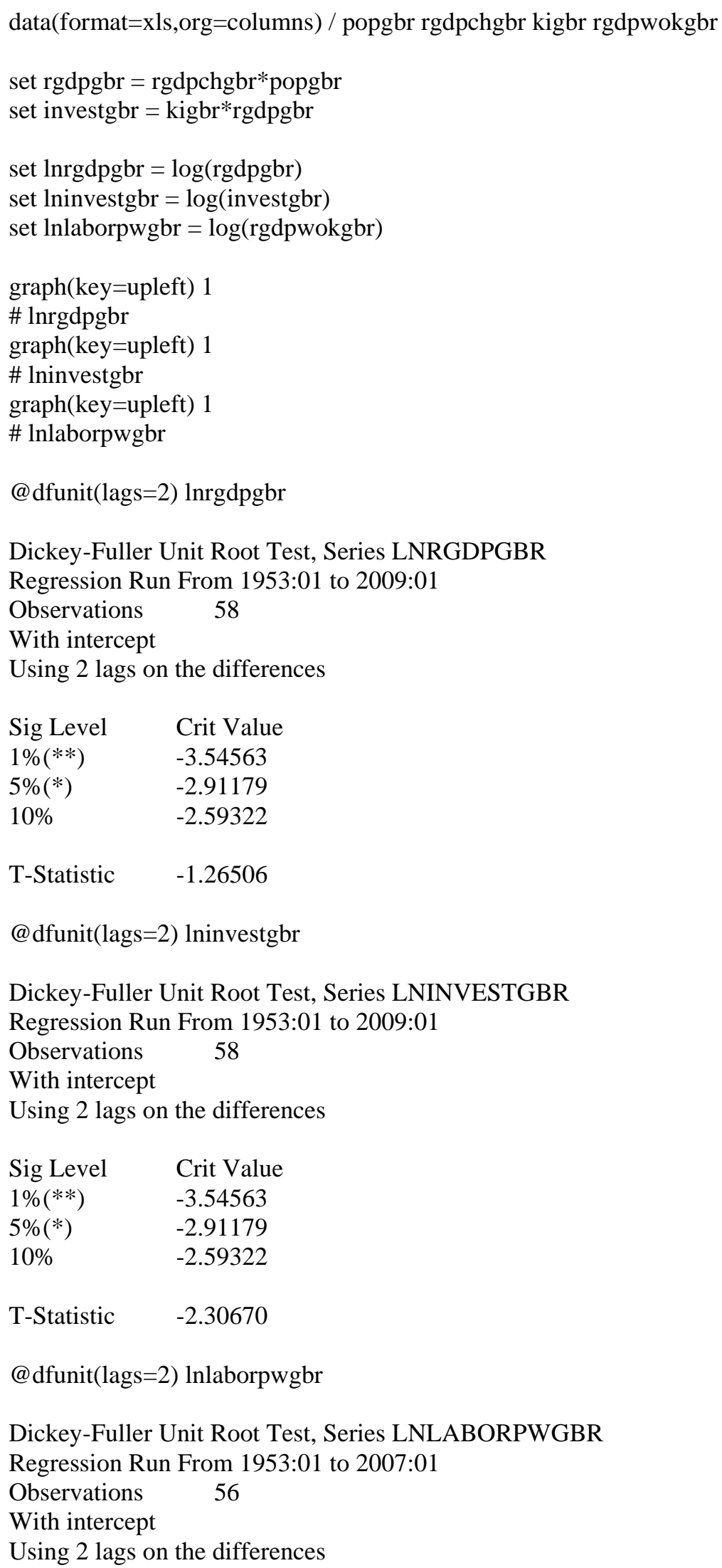




\begin{tabular}{|c|c|c|c|c|c|}
\hline $\begin{array}{l}\text { Sig Leve } \\
1 \%(* *) \\
5 \%(*) \\
10 \%\end{array}$ & & $\begin{array}{l}\text { t Value } \\
54995 \\
1366 \\
99421\end{array}$ & & & \\
\hline T-Statistic & \multicolumn{3}{|c|}{-0.09602} & & \\
\hline \multicolumn{6}{|c|}{ @johmle(lags=2,det=constant,cv=cvector) } \\
\hline \multicolumn{6}{|c|}{$\begin{array}{l}\text { Likelihood Based Analysis of Cointegration } \\
\text { Variables: LNINVESTGBR LNRGDPGBR LNLABORPWGBR } \\
\text { Estimated from 1952:01 to 2007:01 } \\
\text { Data Points } 56 \text { Lags } 2 \text { with Constant }\end{array}$} \\
\hline \multicolumn{6}{|c|}{ Unrestricted eigenvalues and - $\mathrm{T} \log (1-\mathrm{lambda})$} \\
\hline $\begin{array}{r}\text { Rank } \\
0\end{array}$ & & Lambda-max & Trace & Trace-95\% & $\begin{array}{l}\text { LogL } \\
459.7908\end{array}$ \\
\hline 1 & 0.1449 & 8.7680 & 14.8692 & 29.8000 & 464.1748 \\
\hline 2 & 0.1030 & 6.0884 & 6.1013 & 15.4100 & 467.2190 \\
\hline 3 & 0.0002 & 0.0129 & 0.0129 & 3.8400 & 467.2254 \\
\hline
\end{tabular}

Cointegrating Vector for Largest Eigenvalue

LNINVESTGBR LNRGDPGBR LNLABORPWGBR

$9.8288859 .673948 \quad-26.371862$

equation(coeffs=cvector) ecteq *

\# lninvestgbr lnrgdpgbr lnlaborpwgbr

system(model=ectmodel)

variables lninvestgbr lnrgdpgbr lnlaborpwgbr

lags 1 to 2

det constant

ect ecteq

end(system)

estimate

VAR/System - Estimation by Cointegrated Least Squares

Dependent Variable LNINVESTGBR

Annual Data From 1952:01 To 2007:01

Usable Observations $56 \quad$ Degrees of Freedom 51

Mean of Dependent Variable $\quad 0.0343635991$

Std Error of Dependent Variable $\quad 0.0797253660$

Standard Error of Estimate $\quad 0.0732815528$

Sum of Squared Residuals $\quad 0.2738794850$

Durbin-Watson Statistic $\quad 1.496840$

$\begin{array}{lllll}\text { Variable } & \text { Coeff } & \text { Std Error } & \text { T-Stat } & \text { Signif } \\ * * * * * * * * * * * * * * * * * * * * * * * * * * * * * * * * * * * * * * * * * * * * * * * * * * * * * * * * * * * * * * * * * * * * * * * * * * * * * * * * * * * & \\ \text { 1. D_LNINVESTGBR(1) } & -0.454789087 & 0.179127864 & -2.53891 & 0.01421353 \\ \text { 2. D_LNRGDPGBR(1) } & 1.399852952 & 1.614571920 & 0.86701 & 0.38999658 \\ \text { 3. D_LNLABORPWGBR(1) } & 1.594989011 & 1.574237113 & 1.01318 & 0.31575595 \\ \text { 4. Constant } & 3.491767336 & 1.485020530 & 2.35133 & 0.02260860 \\ \text { 5. EC1 }\{1\} & -0.023069042 & 0.009792659 & -2.35575 & 0.02236804\end{array}$


Dependent Variable LNRGDPGBR

Annual Data From 1952:01 To 2007:01

$\begin{array}{lc}\text { Usable Observations } 56 & \text { Degrees of Freedom } 51 \\ \text { Mean of Dependent Variable } & 0.0248296096 \\ \text { Std Error of Dependent Variable } & 0.0165518948 \\ \text { Standard Error of Estimate } & 0.0154752011 \\ \text { Sum of Squared Residuals } & 0.0122135744 \\ \text { Durbin-Watson Statistic } & 1.821677\end{array}$

\begin{tabular}{lllll}
\multicolumn{1}{c}{ Variable } & Coeff & Std Error & T-Stat & Signif \\
$* * * * * * * * * * * * * * * * * * * * * * * * * * * * * * * * * * * * * * * * * * * * * * * * * * * * * * * * * * * * * * * * * * * * * * * * * * * * * * * * * *$ & \\
1. D_LNINVESTGBR(1) & -0.092500645 & 0.037827251 & -2.44534 & 0.01796356 \\
2. D_LNRGDPGBR(1) & 0.365796642 & 0.340956547 & 1.07285 & 0.28838695 \\
3. D_LNLABORPWGBR(1) & 0.339110658 & 0.332438861 & 1.02007 & 0.31251029 \\
4. Constant & 0.386580545 & 0.313598587 & 1.23272 & 0.22333111 \\
5. EC1 $\{1\}$ & -0.002462106 & 0.002067961 & -1.19060 & 0.23932448
\end{tabular}

Dependent Variable LNLABORPWGBR

Annual Data From 1952:01 To 2007:01

Usable Observations $56 \quad$ Degrees of Freedom 51

Mean of Dependent Variable $\quad 0.0205415669$

Std Error of Dependent Variable $\quad 0.0154977316$

Standard Error of Estimate $\quad 0.0148039977$

Sum of Squared Residuals $\quad 0.0111770757$

Durbin-Watson Statistic $\quad 1.930390$

\begin{tabular}{|c|c|c|c|c|}
\hline Variable & Coeff & Std Error & T-Stat $\mathrm{S}$ & ignif \\
\hline 1. D_LNINVESTGBR(1) & -0.081720078 & 0.036186576 & -2.25830 & 0.02823440 \\
\hline 2. D_LNRGDPGBR(1) & -0.116809840 & 0.326168293 & -0.35813 & 0.72172598 \\
\hline 3. D_LNLABORPWGBR(1) & 0.628107043 & 0.318020043 & 1.97505 & 0.05368674 \\
\hline 4. Constant & 0.191621275 & 0.299996925 & 0.63874 & 0.52584771 \\
\hline 5. EC1 $\{1\}$ & -0.001170886 & 0.001978267 & -0.59187 & 0.55654957 \\
\hline
\end{tabular}

compute sigma $=\%$ sigma

errors $($ model $=$ ectmodel,steps $=24$ )

Decomposition of Variance for Series LNINVESTGBR

$\begin{array}{rcrcc}\text { Step } & \text { Std Error } & \text { LNINVESTGBR } & \text { LNRGDPGBR } & \text { LNLABORPWGBR } \\ 1 & 0.06993358 & 100.000 & 0.000 & 0.000 \\ 2 & 0.09626373 & 89.645 & 8.793 & 1.562 \\ 3 & 0.11412664 & 84.062 & 12.428 & 3.511 \\ 4 & 0.12673268 & 80.604 & 14.062 & 5.334 \\ 5 & 0.13631516 & 78.203 & 14.861 & 6.937 \\ 6 & 0.14407346 & 76.304 & 15.332 & 8.364 \\ 7 & 0.15064448 & 74.673 & 15.657 & 9.670 \\ 8 & 0.15638632 & 73.208 & 15.904 & 10.888 \\ 9 & 0.16151889 & 71.861 & 16.100 & 12.038 \\ 10 & 0.16618860 & 70.611 & 16.259 & 13.130 \\ 11 & 0.17049845 & 69.440 & 16.390 & 14.170 \\ 12 & 0.17452346 & 68.342 & 16.499 & 15.160 \\ 13 & 0.17831936 & 67.306 & 16.590 & 16.103 \\ 14 & 0.18192824 & 66.329 & 16.669 & 17.002 \\ 15 & 0.18538226 & 65.405 & 16.736 & 17.859\end{array}$




$\begin{array}{lllll}16 & 0.18870633 & 64.530 & 16.795 & 18.675 \\ 17 & 0.19191994 & 63.701 & 16.846 & 19.452 \\ 18 & 0.19503852 & 62.915 & 16.892 & 20.193 \\ 19 & 0.19807440 & 62.167 & 16.933 & 20.900 \\ 20 & 0.20103752 & 61.457 & 16.970 & 21.573 \\ 21 & 0.20393599 & 60.781 & 17.003 & 22.215 \\ 22 & 0.20677645 & 60.138 & 17.034 & 22.828 \\ 23 & 0.20956439 & 59.525 & 17.062 & 23.413 \\ 24 & 0.21230441 & 58.941 & 17.088 & 23.971\end{array}$

Decomposition of Variance for Series LNRGDPGBR

$\begin{array}{rcccc}\text { Step } & \text { Std Error } & \text { LNINVESTGBR } & \text { LNRGDPGBR } & \text { LNLABORPWGBR } \\ 1 & 0.01476819 & 60.141 & 39.859 & 0.000 \\ 2 & 0.02449194 & 43.423 & 55.766 & 0.811 \\ 3 & 0.03167640 & 37.808 & 60.579 & 1.614 \\ 4 & 0.03727856 & 34.994 & 62.799 & 2.207 \\ 5 & 0.04193852 & 33.326 & 64.901 & 2.903 \\ 7 & 0.04970623 & 31.356 & 65.529 & 3.114 \\ 8 & 0.05310533 & 30.693 & 66.029 & 3.277 \\ 9 & 0.05627643 & 30.149 & 66.790 & 3.519 \\ 11 & 0.06208733 & 29.299 & 67.088 & 3.612 \\ 12 & 0.06477982 & 28.960 & 67.348 & 3.692 \\ 13 & 0.06735593 & 28.662 & 67.575 & 3.763 \\ 14 & 0.06983007 & 28.399 & 67.777 & 3.824 \\ 15 & 0.07221393 & 28.165 & 67.956 & 3.879 \\ 16 & 0.07451714 & 27.955 & 68.117 & 3.928 \\ 17 & 0.07674774 & 27.766 & 68.262 & 3.972 \\ 18 & 0.07891249 & 27.595 & 68.393 & 4.012 \\ 19 & 0.08101717 & 27.439 & 68.513 & 4.048 \\ 20 & 0.08306673 & 27.297 & 68.622 & 4.081 \\ 21 & 0.08506547 & 27.167 & 68.722 & 4.111 \\ 22 & 0.08701715 & 27.048 & 68.813 & 4.164 \\ 24 & 0.09079215 & 26.837 & 68.976 & 4.188\end{array}$

Decomposition of Variance for Series LNLABORPWGBR

$\begin{array}{rlccc}\text { Step } & \text { Std Error } & \text { LNINVESTGBR } & \text { LNRGDPGBR } & \text { LNLABORPWGBR } \\ 1 & 0.01412766 & 53.192 & 31.870 & 14.938 \\ 2 & 0.02257110 & 37.116 & 40.925 & 21.959 \\ 3 & 0.02863200 & 32.473 & 41.204 & 26.323 \\ 4 & 0.03345447 & 30.650 & 40.435 & 28.914 \\ 5 & 0.03762348 & 29.966 & 39.663 & 30.371 \\ 6 & 0.04141377 & 29.781 & 39.063 & 31.156 \\ 7 & 0.04494623 & 29.825 & 38.614 & 31.561 \\ 8 & 0.04827740 & 29.974 & 38.272 & 31.753 \\ 9 & 0.05143903 & 30.172 & 38.003 & 31.826 \\ 10 & 0.05445310 & 30.388 & 37.783 & 31.829 \\ 11 & 0.05733676 & 30.608 & 37.600 & 31.792 \\ 12 & 0.06010410 & 30.823 & 37.445 & 31.732 \\ 13 & 0.06276684 & 31.028 & 37.312 & 31.660 \\ 14 & 0.06533491 & 31.222 & 37.197 & 31.581 \\ 15 & 0.06781677 & 31.403 & 37.096 & 31.501 \\ 16 & 0.07021971 & 31.572 & 37.007 & 31.422 \\ 17 & 0.07255010 & 31.728 & 36.927 & 31.344 \\ 18 & 0.07481354 & 31.873 & 36.857 & 31.270 \\ 19 & 0.07701495 & 32.007 & 36.793 & 31.200\end{array}$




$\begin{array}{lllll}20 & 0.07915877 & 32.131 & 36.736 & 31.133 \\ 21 & 0.08124892 & 32.245 & 36.684 & 31.071 \\ 22 & 0.08328895 & 32.351 & 36.637 & 31.012 \\ 23 & 0.08528206 & 32.450 & 36.593 & 30.957 \\ 24 & 0.08723115 & 32.541 & 36.554 & 30.906\end{array}$

compute nsteps $=12$

compute [vect[strings]] implabel=\| \$

"British Investment",\$

"British GDP",\$

"British Labor Productivity"||

grparm(nopatterns)

grparm(bold) header 36

grparm axislabeling 27

grparm keylabeling 27

@VARIRF(model=ectmodel,steps=nsteps,vlables=implabel,byshocks,byvariables)

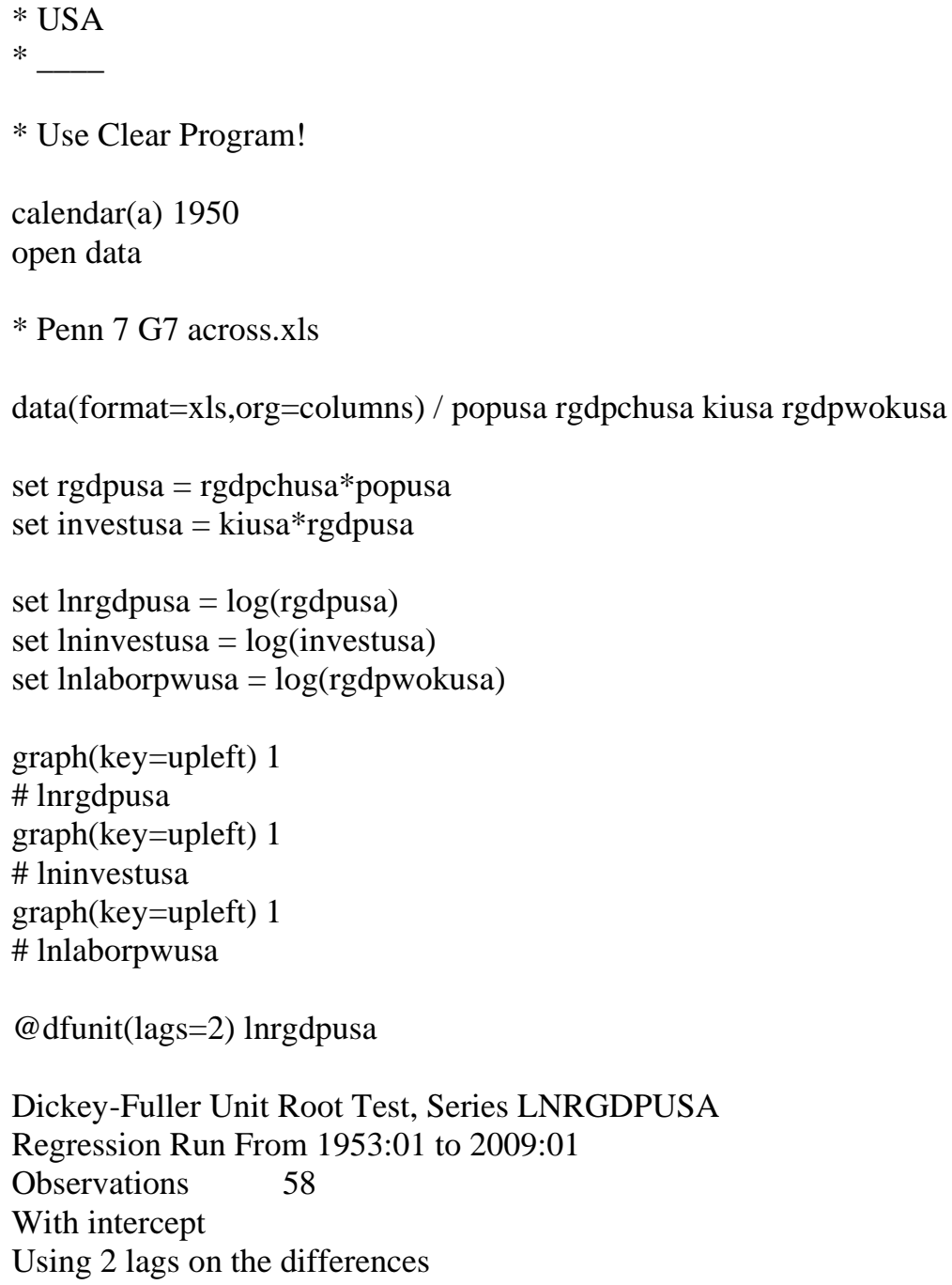




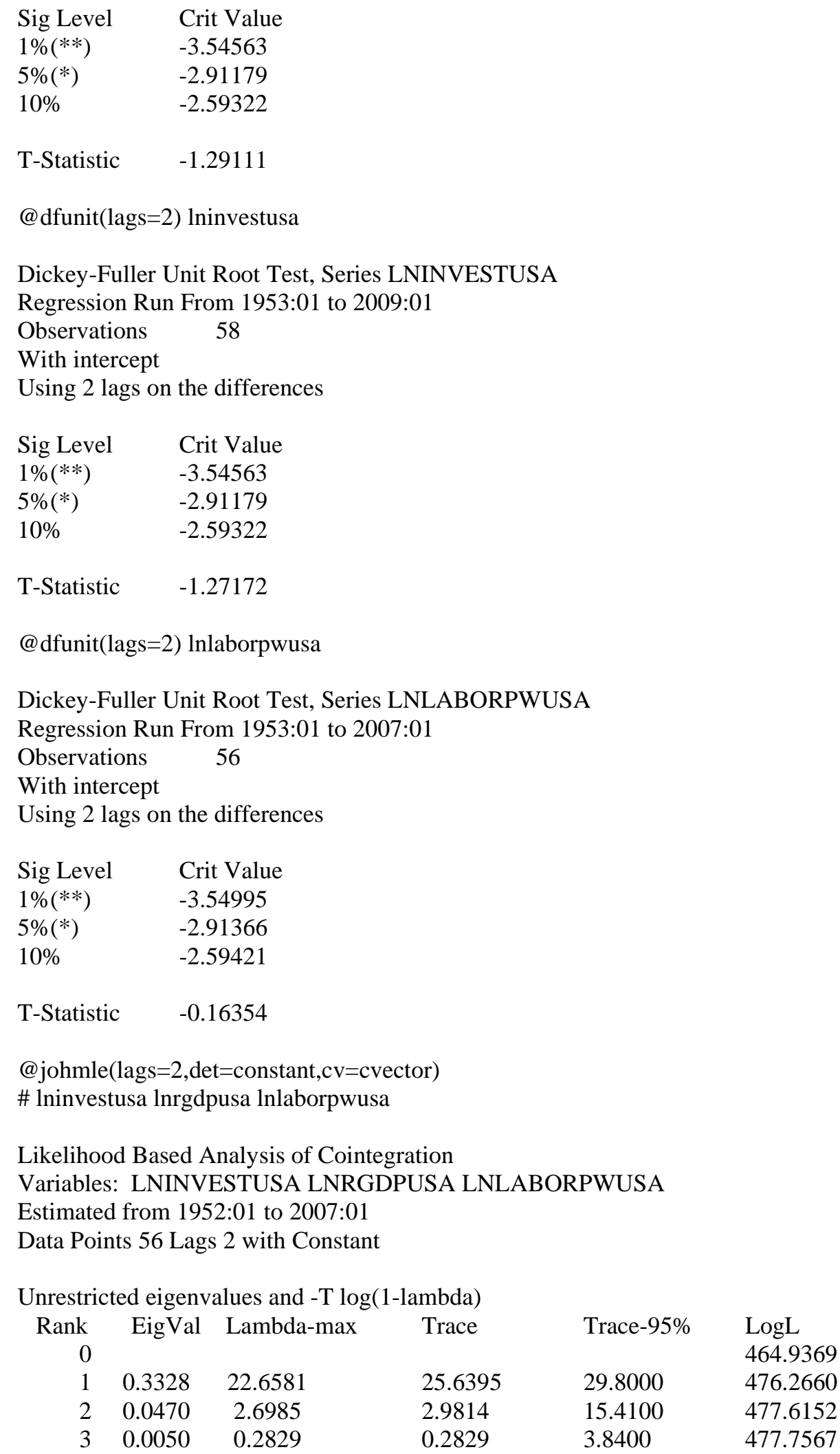

Cointegrating Vector for Largest Eigenvalue LNINVESTUSA LNRGDPUSA LNLABORPWUSA 
equation(coeffs=cvector) ecteq *

\# lninvestusa lnrgdpusa lnlaborpwusa

system(model=ectmodel)

variables lninvestusa lnrgdpusa lnlaborpwusa

lags 1 to 2

det constant

ect ecteq

end(system)

estimate

VAR/System - Estimation by Cointegrated Least Squares

Dependent Variable LNINVESTUSA

Annual Data From 1952:01 To 2007:01

Usable Observations $56 \quad$ Degrees of Freedom 51

Mean of Dependent Variable $\quad 0.0363094515$

Std Error of Dependent Variable $\quad 0.0800793332$

Standard Error of Estimate $\quad 0.0712622406$

Sum of Squared Residuals $\quad 0.2589936537$

Durbin-Watson Statistic 2.009029

$\begin{array}{lllll}\text { Variable } & \text { Coeff } & \text { Std Error } & \text { T-Stat } & \text { Signif } \\ \text { ********************************************************************************** } & \\ \text { 1. D_LNINVESTUSA(1) } & 0.246987482 & 0.196117470 & 1.25939 & 0.21362458 \\ \text { 2. D_LNRGDPUSA(1) } & -1.983074590 & 1.657417140 & -1.19648 & 0.23704013 \\ \text { 3. D_LNLABORPWUSA(1) } & 1.875160484 & 1.486717127 & 1.26128 & 0.21294840 \\ \text { 4. Constant } & -1.478994561 & 0.425908895 & -3.47256 & 0.00105954 \\ \text { 5. EC1\{1\} } & -0.035302289 & 0.009522817 & -3.70713 & 0.00051734\end{array}$

Dependent Variable LNRGDPUSA

Annual Data From 1952:01 To 2007:01

Usable Observations $56 \quad$ Degrees of Freedom 51

Mean of Dependent Variable $\quad 0.0320460920$

Std Error of Dependent Variable $\quad 0.0217043942$

Standard Error of Estimate $\quad 0.0215259643$

Sum of Squared Residuals $\quad 0.0236317242$

Durbin-Watson Statistic $\quad 2.140210$

\begin{tabular}{|c|c|c|c|c|}
\hline Variable & Coeff & Std Error & T-Stat & Signif \\
\hline 1. D LNINVESTUSA(1) & -0.002974929 & 0059240597 & כ20500 0- & 096014504 \\
\hline 2. D_LNRGDPUSA(1) & -0.155967527 & 0.500650863 & -0.31153 & 0.75666805 \\
\hline 3. D_LNLABORPWUSA(1) & 0.396566778 & 0.449088038 & 0.88305 & 0.38135336 \\
\hline 4. Constant & -0.202660980 & 0.128652981 & -1.57525 & 0.12138172 \\
\hline 5. EC1 $1\{1\}$ & -0.005344584 & 0.002876528 & -1.85800 & 0.06894625 \\
\hline
\end{tabular}

Dependent Variable LNLABORPWUSA

Annual Data From 1952:01 To 2007:01

Usable Observations $56 \quad$ Degrees of Freedom 51

Mean of Dependent Variable $\quad 0.0166031446$

Std Error of Dependent Variable $\quad 0.0216666220$

Standard Error of Estimate $\quad 0.0209508664$ 
Sum of Squared Residuals

Durbin-Watson Statistic
0.0223858789

2.218495

\begin{tabular}{|c|c|c|c|c|}
\hline Variable & Coeff & Std Error & T-Stat & Signif \\
\hline 1. D_LNINVESTUSA $(1$ & -0.01012785 & 0.057657897 & -0.17565 & 0.86126111 \\
\hline 2. D_LNRGDPUSA(1) & -0.864230963 & 0.487275235 & -1.77360 & 0.08210021 \\
\hline 3. D_LNLABORPWUSA(1) & 1.020883262 & 0.437089988 & 2.33564 & 0.02348082 \\
\hline 4. Constant & -0.136241202 & 0.125215827 & -1.08805 & 0.28168786 \\
\hline 5. $\operatorname{EC} 1\{1\}$ & -0.003758972 & 0.002799677 & -1.34264 & 0.18533272 \\
\hline
\end{tabular}

compute sigma $=\%$ sigma

errors(model=ectmodel,steps=24)

Decomposition of Variance for Series LNINVESTUSA

$\begin{array}{rrrcc}\text { Step } & \text { Std Error } & \text { LNINVESTUSA } & \text { LNRGDPUSA } & \text { LNLABORPWUSA } \\ 1 & 0.06800652 & 100.000 & 0.000 & 0.000 \\ 2 & 0.08748588 & 97.111 & 1.350 & 1.540 \\ 3 & 0.09512061 & 90.834 & 4.604 & 4.562 \\ 4 & 0.10040413 & 84.543 & 8.092 & 7.365 \\ 5 & 0.10519506 & 79.818 & 10.812 & 9.370 \\ 6 & 0.10999146 & 76.451 & 12.772 & 10.777 \\ 7 & 0.11479527 & 73.870 & 14.264 & 11.866 \\ 8 & 0.11946153 & 71.692 & 15.510 & 12.798 \\ 9 & 0.12393223 & 69.772 & 16.602 & 13.626 \\ 10 & 0.12822323 & 68.073 & 17.566 & 14.360 \\ 11 & 0.13236623 & 66.579 & 18.415 & 15.006 \\ 12 & 0.13638395 & 65.262 & 19.163 & 15.575 \\ 13 & 0.14028910 & 64.093 & 19.826 & 16.081 \\ 14 & 0.14408975 & 63.048 & 20.419 & 16.533 \\ 15 & 0.14779285 & 62.105 & 20.953 & 16.942 \\ 16 & 0.15140518 & 61.251 & 21.437 & 17.312 \\ 17 & 0.15493316 & 60.474 & 21.877 & 17.648 \\ 18 & 0.15838253 & 59.764 & 22.280 & 17.956 \\ 19 & 0.16175838 & 59.113 & 22.649 & 18.238 \\ 20 & 0.16506523 & 58.513 & 22.988 & 18.498 \\ 21 & 0.16830712 & 57.959 & 23.302 & 18.739 \\ 22 & 0.17148774 & 57.446 & 23.593 & 18.961 \\ 23 & 0.17461042 & 56.969 & 23.863 & 19.168 \\ 24 & 0.17767824 & 56.525 & 24.115 & 19.360\end{array}$

Decomposition of Variance for Series LNRGDPUSA

$\begin{array}{rcccc}\text { Step } & \text { Std Error } & \text { LNINVESTUSA } & \text { LNRGDPUSA } & \text { LNLABORPWUSA } \\ 1 & 0.02054252 & 78.554 & 21.446 & 0.000 \\ 2 & 0.03038450 & 69.947 & 29.556 & 0.497 \\ 3 & 0.03697891 & 62.093 & 36.670 & 1.237 \\ 4 & 0.04222712 & 56.766 & 41.423 & 1.811 \\ 5 & 0.04685592 & 53.708 & 44.116 & 2.175 \\ 6 & 0.05112029 & 52.035 & 45.548 & 2.417 \\ 7 & 0.05508892 & 51.027 & 46.370 & 2.603 \\ 8 & 0.05879087 & 50.310 & 46.929 & 2.761 \\ 9 & 0.06226181 & 49.744 & 47.357 & 2.898 \\ 10 & 0.06554009 & 49.286 & 47.698 & 3.017 \\ 11 & 0.06865771 & 48.912 & 47.970 & 3.118 \\ 12 & 0.07163831 & 48.606 & 48.190 & 3.204\end{array}$




$\begin{array}{lllll}13 & 0.07449916 & 48.350 & 48.371 & 3.279 \\ 14 & 0.07725366 & 48.132 & 48.523 & 3.345 \\ 15 & 0.07991280 & 47.944 & 48.654 & 3.402 \\ 16 & 0.08248589 & 47.779 & 48.768 & 3.453 \\ 17 & 0.08498087 & 47.634 & 48.868 & 3.498 \\ 18 & 0.08740451 & 47.505 & 48.957 & 3.538 \\ 19 & 0.08976264 & 47.390 & 49.036 & 3.574 \\ 20 & 0.09206033 & 47.286 & 49.107 & 3.607 \\ 21 & 0.09430202 & 47.192 & 49.171 & 3.637 \\ 22 & 0.09649161 & 47.106 & 49.230 & 3.664 \\ 23 & 0.09863259 & 47.028 & 49.283 & 3.689 \\ 24 & 0.10072806 & 46.956 & 49.332 & 3.711\end{array}$

Decomposition of Variance for Series LNLABORPWUSA

Step Std Error LNINVESTUSA LNRGDPUSA LNLABORPWUSA

$\begin{array}{lllll}1 & 0.01999369 & 72.114 & 22.966 & 4.919\end{array}$

$\begin{array}{lllll}2 & 0.02933262 & 59.499 & 28.310 & 12.191\end{array}$

$\begin{array}{lllll}3 & 0.03593557 & 49.877 & 30.757 & 19.366\end{array}$

$\begin{array}{lllll}4 & 0.04139108 & 44.280 & 30.728 & 24.992\end{array}$

$\begin{array}{lllll}5 & 0.04630259 & 41.472 & 29.417 & 29.112\end{array}$

$\begin{array}{lllll}6 & 0.05089089 & 40.090 & 27.720 & 32.190\end{array}$

$\begin{array}{lllll}7 & 0.05520133 & 39.276 & 26.098 & 34.626\end{array}$

$\begin{array}{lllll}8 & 0.05924848 & 38.671 & 24.695 & 36.634\end{array}$

$\begin{array}{lllll}9 & 0.06305966 & 38.175 & 23.511 & 38.314\end{array}$

$\begin{array}{lllll}10 & 0.06666796 & 37.768 & 22.510 & 39.722\end{array}$

$\begin{array}{lllll}11 & 0.07010232 & 37.438 & 21.657 & 40.906\end{array}$

$\begin{array}{lllll}12 & 0.07338503 & 37.167 & 20.925 & 41.908\end{array}$

$\begin{array}{lllll}13 & 0.07653327 & 36.942 & 20.293 & 42.765\end{array}$

$\begin{array}{lllll}14 & 0.07956102 & 36.751 & 19.745 & 43.504\end{array}$

$\begin{array}{lllll}15 & 0.08248021 & 36.586 & 19.266 & 44.148\end{array}$

$\begin{array}{lllll}16 & 0.08530116 & 36.443 & 18.846 & 44.712\end{array}$

$\begin{array}{lllll}17 & 0.08803284 & 36.317 & 18.474 & 45.209\end{array}$

$\begin{array}{lllll}18 & 0.09068299 & 36.205 & 18.143 & 45.652\end{array}$

$\begin{array}{lllll}19 & 0.09325835 & 36.106 & 17.847 & 46.047\end{array}$

$\begin{array}{lllll}20 & 0.09576481 & 36.017 & 17.581 & 46.402\end{array}$

$\begin{array}{lllll}21 & 0.09820753 & 35.936 & 17.341 & 46.723\end{array}$

$\begin{array}{lllll}22 & 0.10059109 & 35.864 & 17.123 & 47.013\end{array}$

$\begin{array}{lllll}23 & 0.10291956 & 35.797 & 16.924 & 47.279\end{array}$

$\begin{array}{lllll}24 & 0.10519657 & 35.737 & 16.742 & 47.521\end{array}$

compute nsteps $=12$

compute [vect[strings]] implabel=\| \$

"american Investment",\$

"American GDP",\$

"American Labor Productivity"||

grparm(nopatterns)

grparm(bold) header 36

grparm axislabeling 27

grparm keylabeling 27

@VARIRF(model=ectmodel,steps=nsteps,vlables=implabel,byshocks,byvariables) 
Figure A-1. Responses of Labor Productivity in the G7 Countries and Australia Using VECMs

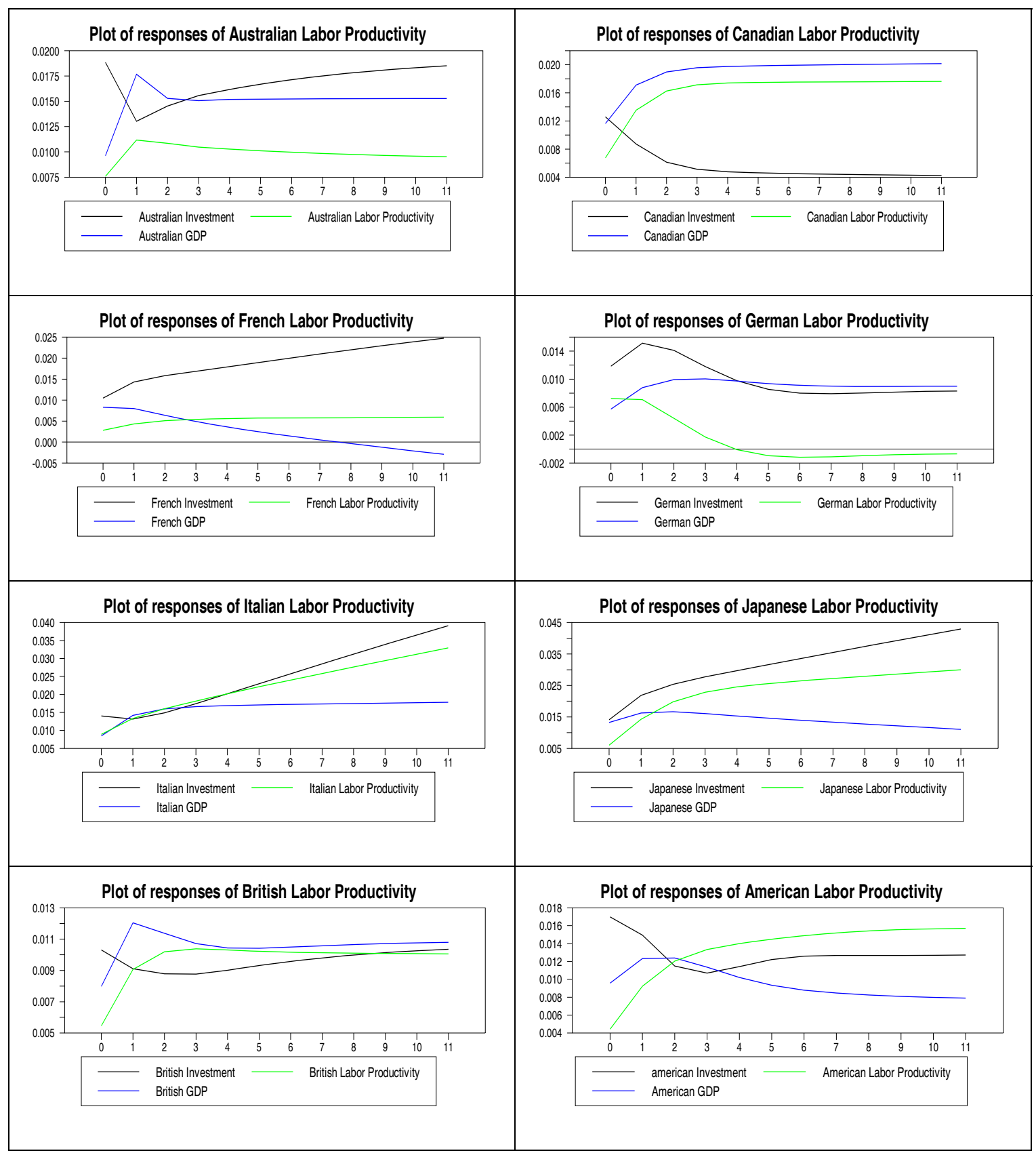




\begin{tabular}{|c|c|c|}
\hline \multicolumn{3}{|c|}{$\begin{array}{l}\text { ECONOMICS DISCUSSION PAPERS } \\
2009\end{array}$} \\
\hline DP NUMBER & AUTHORS & TITLE \\
\hline 09.01 & Le, A.T. & $\begin{array}{l}\text { ENTRY INTO UNIVERSITY: ARE THE CHILDREN OF } \\
\text { IMMIGRANTS DISADVANTAGED? }\end{array}$ \\
\hline 09.02 & Wu, Y. & $\begin{array}{l}\text { CHINA’S CAPITAL STOCK SERIES BY REGION AND } \\
\text { SECTOR }\end{array}$ \\
\hline 09.03 & Chen, M.H. & $\begin{array}{l}\text { UNDERSTANDING WORLD COMMODITY PRICES } \\
\text { RETURNS, VOLATILITY AND DIVERSIFACATION }\end{array}$ \\
\hline 09.04 & Velagic, R. & UWA DISCUSSION PAPERS IN ECONOMICS: THE FIRST 650 \\
\hline 09.05 & McLure, M. & $\begin{array}{l}\text { ROYALTIES FOR REGIONS: ACCOUNTABILITY AND } \\
\text { SUSTAINABILITY }\end{array}$ \\
\hline 09.06 & Chen, A. and Groenewold, N. & $\begin{array}{l}\text { REDUCING REGIONAL DISPARITIES IN CHINA: AN } \\
\text { EVALUATION OF ALTERNATIVE POLICIES }\end{array}$ \\
\hline 09.07 & Groenewold, N. and Hagger, A. & $\begin{array}{l}\text { THE REGIONAL ECONOMIC EFFECTS OF IMMIGRATION: } \\
\text { SIMULATION RESULTS FROM A SMALL CGE MODEL. }\end{array}$ \\
\hline 09.08 & Clements, K. and Chen, D. & AFFLUENCE AND FOOD: SIMPLE WAY TO INFER INCOMES \\
\hline 09.09 & Clements, K. and Maesepp, M. & A SELF-REFLECTIVE INVERSE DEMAND SYSTEM \\
\hline 09.10 & Jones, C. & $\begin{array}{l}\text { MEASURING WESTERN AUSTRALIAN HOUSE PRICES: } \\
\text { METHODS AND IMPLICATIONS }\end{array}$ \\
\hline 09.11 & Siddique, M.A.B. & $\begin{array}{l}\text { WESTERN AUSTRALIA-JAPAN MINING CO-OPERATION: AN } \\
\text { HISTORICAL OVERVIEW }\end{array}$ \\
\hline 09.12 & Weber, E.J. & $\begin{array}{l}\text { PRE-INDUSTRIAL BIMETALLISM: THE INDEX COIN } \\
\text { HYPTHESIS }\end{array}$ \\
\hline 09.13 & McLure, M. & $\begin{array}{l}\text { PARETO AND PIGOU ON OPHELIMITY, UTILITY AND } \\
\text { WELFARE: IMPLICATIONS FOR PUBLIC FINANCE }\end{array}$ \\
\hline 09.14 & Weber, E.J. & $\begin{array}{l}\text { WILFRED EDWARD GRAHAM SALTER: THE MERITS OF A } \\
\text { CLASSICAL ECONOMIC EDUCATION }\end{array}$ \\
\hline 09.15 & Tyers, R. and Huang, L. & $\begin{array}{l}\text { COMBATING CHINA’S EXPORT CONTRACTION: FISCAL } \\
\text { EXPANSION OR ACCELERATED INDUSTRIAL REFORM }\end{array}$ \\
\hline 09.16 & $\begin{array}{l}\text { Zweifel, P., Plaff, D. and } \\
\text { Kühn, J. }\end{array}$ & $\begin{array}{l}\text { IS REGULATING THE SOLVENCY OF BANKS COUNTER- } \\
\text { PRODUCTIVE? }\end{array}$ \\
\hline 09.17 & Clements, K. & THE PHD CONFERENCE REACHES ADULTHOOD \\
\hline 09.18 & McLure, M. & $\begin{array}{l}\text { THIRTY YEARS OF ECONOMICS: UWA AND THE WA } \\
\text { BRANCH OF THE ECONOMIC SOCIETY FROM } 1963 \text { TO } 1992\end{array}$ \\
\hline 09.19 & Harris, R.G. and Robertson, P. & $\begin{array}{l}\text { TRADE, WAGES AND SKILL ACCUMULATION IN THE } \\
\text { EMERGING GIANTS }\end{array}$ \\
\hline 09.20 & $\begin{array}{l}\text { Peng, J., Cui, J., Qin, F. and } \\
\text { Groenewold, N. }\end{array}$ & STOCK PRICES AND THE MACRO ECONOMY IN CHINA \\
\hline 09.21 & Chen, A. and Groenewold, N. & $\begin{array}{l}\text { REGIONAL EQUALITY AND NATIONAL DEVELOPMENT IN } \\
\text { CHINA: IS THERE A TRADE-OFF? }\end{array}$ \\
\hline
\end{tabular}




\begin{tabular}{|c|c|c|}
\hline \multicolumn{3}{|c|}{$\begin{array}{l}\text { ECONOMICS DISCUSSION PAPERS } \\
2010\end{array}$} \\
\hline DP NUMBER & AUTHORS & TITLE \\
\hline 10.01 & Hendry, D.F. & $\begin{array}{l}\text { RESEARCH AND THE ACADEMIC: A TALE OF } \\
\text { TWO CULTURES }\end{array}$ \\
\hline 10.02 & $\begin{array}{l}\text { McLure, M., Turkington, D. and Weber, } \\
\text { E.J. }\end{array}$ & A CONVERSATION WITH ARNOLD ZELLNER \\
\hline 10.03 & $\begin{array}{l}\text { Butler, D.J., Burbank, V.K. and } \\
\text { Chisholm, J.S. }\end{array}$ & $\begin{array}{l}\text { THE FRAMES BEHIND THE GAMES: PLAYER'S } \\
\text { PERCEPTIONS OF PRISONER'S DILEMMA, } \\
\text { CHICKEN, DICTATOR, AND ULTIMATUM GAMES }\end{array}$ \\
\hline 10.04 & Harris, R.G., Robertson, P.E. and Xu, J.Y. & $\begin{array}{l}\text { THE INTERNATIONAL EFFECTS OF CHINA'S } \\
\text { GROWTH, TRADE AND EDUCATION BOOMS }\end{array}$ \\
\hline 10.05 & Clements, K.W., Mongey, S. and Si, J. & $\begin{array}{l}\text { THE DYNAMICS OF NEW RESOURCE PROJECTS } \\
\text { A PROGRESS REPORT }\end{array}$ \\
\hline 10.06 & Costello, G., Fraser, P. and Groenewold, N. & $\begin{array}{l}\text { HOUSE PRICES, NON-FUNDAMENTAL } \\
\text { COMPONENTS AND INTERSTATE SPILLOVERS: } \\
\text { THE AUSTRALIAN EXPERIENCE }\end{array}$ \\
\hline 10.07 & Clements, $\mathrm{K}$. & $\begin{array}{l}\text { REPORT OF THE } 2009 \text { PHD CONFERENCE IN } \\
\text { ECONOMICS AND BUSINESS }\end{array}$ \\
\hline 10.08 & Robertson, P.E. & $\begin{array}{l}\text { INVESTMENT LED GROWTH IN INDIA: HINDU } \\
\text { FACT OR MYTHOLOGY? }\end{array}$ \\
\hline 10.09 & Fu, D., Wu, Y. and Tang, Y. & $\begin{array}{l}\text { THE EFFECTS OF OWNERSHIP STRUCTURE AND } \\
\text { INDUSTRY CHARACTERISTICS ON EXPORT } \\
\text { PERFORMANCE }\end{array}$ \\
\hline 10.10 & Wu, Y. & $\begin{array}{l}\text { INNOVATION AND ECONOMIC GROWTH IN } \\
\text { CHINA }\end{array}$ \\
\hline 10.11 & Stephens, B.J. & $\begin{array}{l}\text { THE DETERMINANTS OF LABOUR FORCE } \\
\text { STATUS AMONG INDIGENOUS AUSTRALIANS }\end{array}$ \\
\hline 10.12 & Davies, M. & $\begin{array}{l}\text { FINANCING THE BURRA BURRA MINES, SOUTH } \\
\text { AUSTRALIA: LIQUIDITY PROBLEMS AND } \\
\text { RESOLUTIONS }\end{array}$ \\
\hline 10.13 & Tyers, R. and Zhang, Y. & APPRECIATING THE RENMINBI \\
\hline 10.14 & Clements, K.W., Lan, Y. and Seah, S.P. & $\begin{array}{l}\text { THE BIG MAC INDEX TWO DECADES ON } \\
\text { AN EVALUATION OF BURGERNOMICS }\end{array}$ \\
\hline 10.15 & Robertson, P.E. and Xu, J.Y. & $\begin{array}{l}\text { IN CHINA'S WAKE: } \\
\text { HAS ASIA GAINED FROM CHINA'S GROWTH? }\end{array}$ \\
\hline 10.16 & Clements, K.W. and Izan, H.Y. & $\begin{array}{l}\text { THE PAY PARITY MATRIX: A TOOL FOR } \\
\text { ANALYSING THE STRUCTURE OF PAY }\end{array}$ \\
\hline 10.17 & Gao, G. & WORLD FOOD DEMAND \\
\hline 10.18 & Wu, Y. & $\begin{array}{l}\text { INDIGENOUS INNOVATION IN CHINA: } \\
\text { IMPLICATIONS FOR SUSTAINABLE GROWTH }\end{array}$ \\
\hline 10.19 & Robertson, P.E. & DECIPHERING THE HINDU GROWTH EPIC \\
\hline 10.20 & Stevens, G. & $\begin{array}{l}\text { RESERVE BANK OF AUSTRALIA-THE ROLE OF } \\
\text { FINANCE }\end{array}$ \\
\hline 10.21 & Widmer, P.K., Zweifel, P. and Farsi, M. & $\begin{array}{l}\text { ACCOUNTING FOR HETEROGENEITY IN THE } \\
\text { MEASUREMENT OF HOSPITAL PERFORMANCE }\end{array}$ \\
\hline
\end{tabular}




\begin{tabular}{|l|l|l|}
\hline 10.22 & McLure, $\mathrm{M}$. & $\begin{array}{l}\text { ASSESSMENTS OF A. C. PIGOU'S FELLOWSHIP } \\
\text { THESES }\end{array}$ \\
\hline 10.23 & Poon, A.R. & $\begin{array}{l}\text { THE ECONOMICS OF NONLINEAR PRICING: } \\
\text { EVIDENCE FROM AIRFARES AND GROCERY } \\
\text { PRICES }\end{array}$ \\
\hline 10.24 & Halperin, D. & $\begin{array}{l}\text { FORECASTING METALS RETURNS: A BAYESIAN } \\
\text { DECISION THEORETIC APPROACH }\end{array}$ \\
\hline 10.25 & Clements, K.W. and Si. J. & $\begin{array}{l}\text { THE INVESTMENT PROJECT PIPELINE: COST } \\
\text { ESCALATION, LEAD-TIME, SUCCESS, FAILURE } \\
\text { AND SPEED }\end{array}$ \\
\hline 10.26 & Chen, A., Groenewold, N. and Hagger, A.J. & $\begin{array}{l}\text { THE REGIONAL ECONOMIC EFFECTS OF A } \\
\text { REDUCTION IN CARBON EMISSIONS }\end{array}$ \\
\hline 10.27 & $\begin{array}{l}\text { Siddique, A., Selvanathan, E.A. and } \\
\text { Selvanathan, S. }\end{array}$ & $\begin{array}{l}\text { REMITTANCES AND ECONOMIC GROWTH: } \\
\text { EMPIRICAL EVIDENCE FROM BANGLADESH, } \\
\text { INDIA AND SRI LANKA }\end{array}$ \\
\hline
\end{tabular}




\section{ECONOMICS DISCUSSION PAPERS}

2011

\begin{tabular}{|c|c|c|}
\hline DP NUMBER & AUTHORS & TITLE \\
\hline 11.01 & Robertson, P.E. & DEEP IMPACT: CHINA AND THE WORLD ECONOMY \\
\hline 11.02 & Kang, C. and Lee, S.H. & $\begin{array}{l}\text { BEING KNOWLEDGEABLE OR SOCIABLE? } \\
\text { DIFFERENCES IN RELATIVE IMPORTANCE OF } \\
\text { COGNITIVE AND NON-COGNITIVE SKILLS }\end{array}$ \\
\hline 11.03 & Turkington, D. & DIFFERENT CONCEPTS OF MATRIX CALCULUS \\
\hline 11.04 & Golley, J. and Tyers, R. & $\begin{array}{l}\text { CONTRASTING GIANTS: DEMOGRAPHIC CHANGE } \\
\text { AND ECONOMIC PERFORMANCE IN CHINA AND } \\
\text { INDIA }\end{array}$ \\
\hline 11.05 & Collins, J., Baer, B. and Weber, E.J. & $\begin{array}{l}\text { ECONOMIC GROWTH AND EVOLUTION: PARENTAL } \\
\text { PREFERENCE FOR QUALITY AND QUANTITY OF } \\
\text { OFFSPRING }\end{array}$ \\
\hline 11.06 & Turkington, D. & $\begin{array}{l}\text { ON THE DIFFERENTIATION OF THE LOG } \\
\text { LIKELIHOOD FUNCTION USING MATRIX CALCULUS }\end{array}$ \\
\hline 11.07 & Groenewold, N. and Paterson, J.E.H. & $\begin{array}{l}\text { STOCK PRICES AND EXCHANGE RATES IN } \\
\text { AUSTRALIA: ARE COMMODITY PRICES THE } \\
\text { MISSING LINK? }\end{array}$ \\
\hline 11.08 & Chen, A. and Groenewold, N. & $\begin{array}{l}\text { REDUCING REGIONAL DISPARITIES IN CHINA: IS } \\
\text { INVESTMENT ALLOCATION POLICY EFFECTIVE? }\end{array}$ \\
\hline 11.09 & Williams, A., Birch, E. and Hancock , P. & $\begin{array}{l}\text { THE IMPACT OF ON-LINE LECTURE RECORDINGS } \\
\text { ON STUDENT PERFORMANCE }\end{array}$ \\
\hline 11.10 & Pawley, J. and Weber, E.J. & $\begin{array}{l}\text { INVESTMENT AND TECHNICAL PROGRESS IN THE } \\
\text { G7 COUNTRIES AND AUSTRALIA }\end{array}$ \\
\hline
\end{tabular}

